University of Rhode Island

DigitalCommons@URI

Open Access Dissertations

2017

\title{
Asymptotic Stepwise Coupled Modes for Horizontally-Variable Acoustic Media
}

Charles E. White

University of Rhode Island, cewhite83@gmail.com

Follow this and additional works at: https://digitalcommons.uri.edu/oa_diss

\section{Recommended Citation}

White, Charles E., "Asymptotic Stepwise Coupled Modes for Horizontally-Variable Acoustic Media" (2017). Open Access Dissertations. Paper 672.

https://digitalcommons.uri.edu/oa_diss/672

This Dissertation is brought to you for free and open access by DigitalCommons@URI. It has been accepted for inclusion in Open Access Dissertations by an authorized administrator of DigitalCommons@URI. For more information, please contact digitalcommons-group@uri.edu. 


\section{ASYMPTOTIC STEPWISE COUPLED MODES FOR HORIZONTALLY-VARIABLE ACOUSTIC MEDIA \\ BY \\ CHARLES E. WHITE}

A DISSERTATION SUBMITTED IN PARTIAL FULFILLMENT OF THE REQUIREMENTS FOR THE DEGREE OF DOCTOR OF PHILOSOPHY

IN

OCEAN ENGINEERING 
DOCTOR OF PHILOSOPHY DISSERTATION

$\mathrm{OF}$

CHARLES E. WHITE

\title{
APPROVED:
}

Dissertation Committee:

\author{
Major Professor Gopu R. Potty \\ James H. Miller \\ Cathy Ann Clark \\ Nasser H. Zawia \\ DEAN OF THE GRADUATE SCHOOL
}

\section{UNIVERSITY OF RHODE ISLAND}




\begin{abstract}
Efficient and accurate mathematical codes for the prediction of underwater sound propagation are a critical component of SONAR system development and operation. Shallow water provides a unique set of complications to the problem of acoustic propagation prediction due to range dependence of acoustic properties resulting from a high number of wave interactions with the surface and seafloor and bathymetric variation. In this situation, the modes of vibration of the acoustic wave equation become coupled, with a transfer of energy between adjacent modes occurring upon traversing a horizontal change of environment. While mode methods have been developed for solving this range-dependent problem with varying accuracy, the computational intensity of these solutions makes them unsuitable for use in applications where real-time or near real-time performance is desired. The goal of the research presented herein was to develop, implement, and verify an efficient and rigorous coupled-mode solution for acoustic wave propagation in shallow water range-dependent environments. Particular interest was given to developing a solution that maintains analytical integrity while executing in a time window that is useful for tactical applications. A theoretical framework involving a range-expanded inner product for capturing the coupling between modes as they propagate through a horizontally-variable medium is presented. This framework includes a novel discretization of the range-dependent acoustic medium. A difference equations approach, which implements the inner product to account for non-adiabatic energy transfer between modes, is used to recursively compute reflection and transmission coefficients throughout the discretized environment. Increased efficiency is gained in the method due to the ability to compute coupling via closed-form algebraic expressions and in the application of asymptotic analysis to simplify the transmission and reflection coefficient solutions.
\end{abstract}




\section{ACKNOWLEDGMENTS}

A number of people are owed thanks for their contribution toward the completion of this dissertation. First, the author would like to express his sincere gratitude towards Prof. Gopu Potty, Prof. James H. Miller, and Dr. Cathy Ann Clark, members of the Doctoral Program Committee. Their constant encouragement and presentation of challenging questions have greatly influenced the author's growth as a scientist. In addition, Prof. Yang Shen and Dr. Steven Crocker are acknowledged for their feedback and service on the oral examining committees.

The support of the Naval Undersea Warfare Center (NUWC) of Newport, Rhode Island is also acknowledged. In particular, thanks is expressed toward Mr. Neil Dubois, director of the NUWC In-house Laboratory Independent Research Program. Support given to the author by his supervisor, Dr. Stephen E. Turner, Head of the Undersea Modeling Branch of the Ranges, Engineering, \& Analysis Department is also expressly acknowledged.

The author wishes to express his thanks and appreciation to Mr. Roy Deavenport for his input during the literature review process and to Dr. Russell Herman for multiple conversations concerning the solutions to partial differential equations. Dr. Georges Dossot is thanked for his assistance with generating the parabolic equation comparison data. Gratitude also goes to Dr. Marcia Isakson and Ms. Fiona Cheung for their time and effort in providing the three-dimensional finite element data used in the method verification process.

Finally, the author would like to acknowledge Dr. Gustave A. Leibiger. While his passing did not permit direct contact with the author, his work in the area of underwater acoustic propagation prediction served as a guide and inspiration for much of the research performed in the context of this dissertation. 


\section{DEDICATION}

This dissertation is dedicated to the Ancestors and to the Orisha, without which I would be lost, and to my spiritual godparent, Raven Morgaine.

To my partner, Ian Strickland, my mother, Debbie Carter, my uncle, Jeff Carter, and to my sisters at heart, Elizabeth McCullough and Erica Penardo, thank you for your constant love and support through this journey.

To Dr. Gene A. Tagliarini, I thank you for inspiring me to enter the field of academic research as an undergraduate. Your selfless passion for academic instruction and encouragement has impacted my life in a way that cannot be overstated. 


\title{
TABLE OF CONTENTS
}

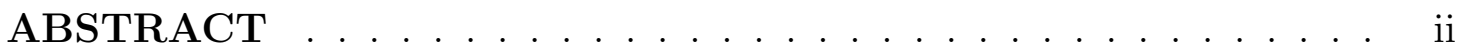

ACKNOWLEDGMENTS ................. . iii

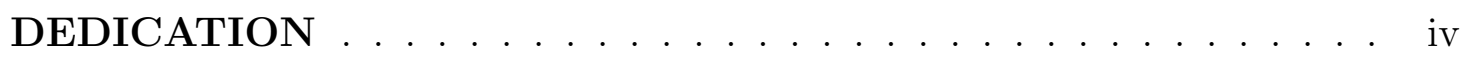

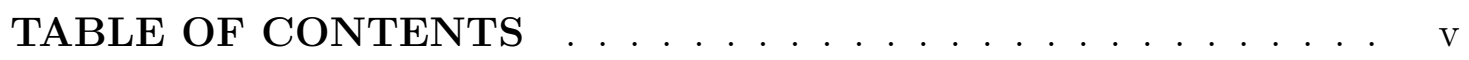

LIST OF FIGURES . . . . . . . . . . . . . . . . . . viii

\section{CHAPTER}

1 Introduction . . . . . . . . . . . . . . . . 1

1.1 Background . . . . . . . . . . . . . . . . 1

1.2 Asymptotic Analysis . . . . . . . . . . . . . . . . 3

1.3 The Acoustic Wave Equation . . . . . . . . . . . . . . . 6

1.4 The Acoustic Velocity Potential . . . . . . . . . . . . . . 12

1.5 The Helmholtz Equation . . . . . . . . . . . . . . . . 15

1.6 Source Representation . . . . . . . . . . . . . . . . . . . 16

1.7 General Solution: Green's Function . . . . . . . . . . . . 20

List of References . . . . . . . . . . . . . . . . . . . . 22

2 Normal Mode Theory . . . . . . . . . . . . . . . . . . 23

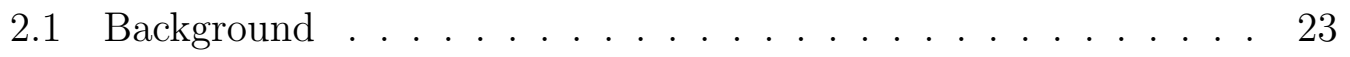

2.2 Solution via Generalized Separation of Variables . . . . . . . . 23

2.3 Wavenumber Decomposition and Evanescent Modes . . . . . . 28

2.4 Modal Velocities . . . . . . . . . . . . . . . . . . . . . 31 


\section{Page}

2.5 Solution via Cauchy's Residue Theorem . . . . . . . . . . . . 33

2.6 Range-Dependent Normal Modes . . . . . . . . . . . . . . . . 36

2.7 Previous Work . . . . . . . . . . . . . . . . 36

List of References . . . . . . . . . . . . . . . . . . . . . . . . . 42

3 Asymptotic Stepwise Coupled Modes . . . . . . . . . . . . . . . 44

3.1 Introduction ..................... . . . 44

3.2 The Range-Expanded Normal Mode Inner Product . . . . . . . 45

3.3 Discretization of the Range-Dependent Medium . . . . . . . . . 50

3.4 Asymptotic Identities . . . . . . . . . . . . . . . . . . . . 57

3.5 Asymptotic Solution . . . . . . . . . . . . . . . 58

3.6 Cylindrical Wave Extension . . . . . . . . . . . . . . . 65

3.7 Reformulation for Numerical Stability . . . . . . . . . . . . 68

3.8 Mode Turnaround and Eigenvalue Lag Distance . . . . . . . . . 71

List of References . . . . . . . . . . . . . . . . . . . 76

4 Verification of Theory . . . . . . . . . . . . . . . . 77

4.1 Background ...................... 77

4.2 Comparison to Benchmark Solution . . . . . . . . . . . . . 78

4.3 Comparison to Parabolic Equation Solution . . . . . . . . . 86

4.4 Comparison to 3D Finite Element Solution . . . . . . . . . . . . 90

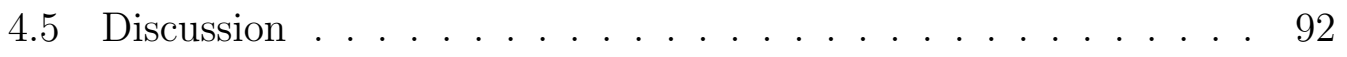

List of References . . . . . . . . . . . . . . . . . . 93

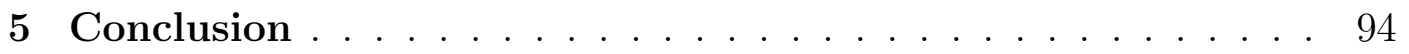

List of References . . . . . . . . . . . . . . . . . . . . . 96 


\section{APPENDIX}

Note on the Effects of Range-Dependent Bathymetry on Modal Group Velocities . . . . . . . . . . . . . . . . . . 97

List of References . . . . . . . . . . . . . . . . . . . . 103

BIBLIOGRAPHY . . . . . . . . . . . . . . . . . . . 104 


\section{LIST OF FIGURES}

$\begin{array}{lll}\text { Figure Page } & \text { Pag }\end{array}$

3.1 Discretization of Horizontally-Variable Bottom Depth . . . . . . 53

3.1a Continuous Environment .......... . 53

3.1b Discretized Environment . . . . . . . . . 53

3.2 Stepwise Discretization of Subenvironment . . . . . . . . . . 54

3.3 Energy Transport Through Discretized Environment . . . . . . 55

$3.4 \quad$ Eigenvalue Lag Distance . . . . . . . . . . . . . . . . . 72

3.5 Effect of Mode Turnaround on Transmission Coefficients . . . . 73

3.5a Grazing Angle Turnover . . . . . . . . . . 73

3.5b Transmission Coefficient Discontinuity . . . . . . 73

3.6 Artificial Peak Correction in Transmission Loss Estimate . . . . 75

3.6a Before Correction ............ . 75

3.6b After Correction .............. 75

4.1 Comparison to Benchmark: Upslope . . . . . . . . . . . 79

4.1a Upslope Scenario . . . . . . . . . . 79

4.1b Transmission Loss Comparison . . . . . . . . . 79

4.2 Comparison to Benchmark: Downslope . . . . . . . . . . 80

4.2a Downslope Scenario . . . . . . . . . . 80

4.2b Transmission Loss Comparison . . . . . . . . 80

4.3 Comparison to Benchmark: Two-slope Uphill . . . . . . . . . . 81

4.3a Two-slope Uphill Scenario . . . . . . . . . . . 81

4.3b Transmission Loss Comparison . . . . . . . . . 81 
4.4 Comparison to Benchmark: Two-slope Downhill . . . . . . . . . 82

4.4a Two-slope Downhill Scenario . . . . . . . . . 82

4.4b Transmission Loss Comparison . . . . . . . . . 82

4.5 Comparison to Benchmark: 2D Seamount .......... 83

4.5a 2D Seamount Scenario ............ 83

4.5b Transmission Loss Comparison . . . . . . . . . 83

4.6 Comparison to Benchmark: 2D Canyon . . . . . . . . . . . . . 84

4.6a 2D Canyon Scenario . . . . . . . . . . . 84

4.6b Transmission Loss Comparison . . . . . . . . . 84

4.7 Comparison to Benchmark: General Multi-slope . . . . . . . . . 85

4.7a General Multi-slope Scenario . . . . . . . . . 85

4.7b Transmission Loss Comparison . . . . . . . . . 85

4.8 Comparison to Parabolic Equation Solution . . . . . . . . . 88

4.8a High Frequency 2D Seamount . . . . . . . 88

4.8b ASCM vs. COUPLE ............ 88

4.8c MMPE vs. COUPLE ............ 88

4.8d MMPE vs. ASCM ............. . 88

$4.9 \quad$ MMPE Pressure Field . . . . . . . . . . . . . . . . 89

4.10 Comparison to 3D Finite Element Solution . . . . . . . . . . . . 91

4.10a COUPLE vs. FEM ................ 91

4.10b ASCM vs. FEM ................ 91

A.1 Effects of Variable Bottom Depth: Mode 1 . . . . . . . . . 100

A.1a Group Velocity Pertubation ............ 100 
Figure

Page

A.1b Grazing Angle Perturbation . . . . . . . . . . 100

A.2 Effects of Variable Bottom Depth: Mode 3 . . . . . . . . . . 101

A.2a Group Velocity Pertubation ........... . 101

A.2b Grazing Angle Perturbation . . . . . . . . . 101

A.3 Effects of Variable Bottom Depth: Mode 7 . . . . . . . . . . 102

A.3a Group Velocity Pertubation . . . . . . . . . . 102

A.3b Grazing Angle Perturbation . . . . . . . . . . 102 


\section{CHAPTER 1}

\section{Introduction}

\section{$1.1 \quad$ Background}

Numerical models have been used as standard research tools in the field of ocean acoustics since the mid-1970s. Technological leaps in the fields of computer science and computer engineering have made it possible for these models to handle complex nonlinear and inter-coupled phenomena. These models have become particularly useful in the discipline of acoustic propagation prediction, where spatially and temporally dynamic acoustic terrain necessitate the simulation of multiple simultaneous physical phenomena to determine how acoustic energy will propagate in the underwater environment. Despite advances in computer microprocessor technology, the use of multiphysics models (such as those used in acoustic propagation prediction) in real-time or near real-time applications still presents a significant challenge due to the computational complexity of these models.

Since the early days of World War II anti-submarine SONAR systems have played a key part in naval operations. Efficient and accurate mathematical codes for the prediction of underwater sound propagation are a critical component of these systems. In deep water regimes, where the acoustic properties of the ocean are slowly varying with horizontal range, the pressure field associated with acoustic phenomena can be approximated by a sum of linearly independent normal modes. A number of efficient and accurate models have been developed around this range-independent solution to acoustic propagation. In shallow water regimes the assumption of range independence fails in environments with rapidly-varying bathymetry and/or sound speed. In this situation, the modes of vibration of the acoustic wave equation become coupled, with a transfer of energy between adjacent modes occurring upon traversing a horizontal change of environment. While 
mode methods have been developed for solving this range-dependent problem with varying accuracy, the computational intensity of these solutions often makes them unsuitable for use in the warfare environment, where tactical constraints dictate the need for more efficient codes.

The goal of the research presented herein was to develop, implement, and verify an efficient and rigorous coupled-mode solution for acoustic wave propagation in range-dependent environments. Particular interest was given to developing a solution that maintains analytical integrity while executing in a time window that is tactically useful for warfare applications. In this dissertation a theoretical framework involving an inner product for capturing the coupling between modes as they propagate through a horizontally-variable medium is presented. A difference equations approach, which implements the inner product to account for non-adiabatic energy transfer between modes, is used to recursively compute reflection and transmission coefficients at discrete interfaces separating stepwise regions throughout the medium.

Increased efficiency is obtained in the presented method by writing the rangedependent eigenfunctions and eigenvalues associated with the acoustic modes of propagation as an expansion in range. This leads to the ability to estimate the inner product via a truncated Taylor series. Asymptotic analysis is then applied to develop a set of asymptotic identities involving the eigenfunctions and eigenvalues in adjacent regions, ultimately leading to simplified expressions for the reflection and transmission coefficients. A novel discretization approach, involving the estimation of the general range-dependent environment via a series of simpler range-dependent subenvironments, results in closed-form expressions for computing non-adiabatic mode coupling. The ultimate payoff of this discretization method is the elimination of the need to numerically compute inner product integrals at each interface 
throughout the discretized environment. Elimination of the need to numerically solve the eigenvalue search problem is an additional computational benefit of this approach.

\subsection{Asymptotic Analysis}

Asymptotic analysis is a method of describing limiting behavior. This form of analysis has applications across many areas of science. In computer science, for example, asymptotic analysis is used to analyze the computational efficiency of specific computing algorithms. In applied mathematics asymptotic analysis is used to derive numerical methods for approximating mathematical functions and equations. Regarding this latter case, the following equivalence relation forms the basis of many such analyses:

$$
\begin{gathered}
f \sim g \\
\text { if and only if } \\
\lim _{n \rightarrow \infty} \frac{f(n)}{g(n)}=1 .
\end{gathered}
$$

This is an equivalence relation on the set of functions of the variable $n$ defined on some domain. The equivalence class of $f$ then consists of all functions $g$ which approximate $f$ in the stated limit.

In addition to the typical mathematical notation from real and complex analysis and calculus, asymptotic analysis has its own family of notation collectively

known as Bachmann-Landau notation. Of particular interest is big-O and little-o notation. In regards to the former, let $f$ and $g$ be two functions defined on some subset of the real numbers. We write:

$$
f(x)=\mathcal{O}[g(x)] \quad \text { as } \quad x \rightarrow \infty,
$$

if and only if there is a positive constant $M$ such that for all sufficiently large values of $x$, the absolute value of $f(x)$ is at most $M$ multiplied by the absolute 
value of $g(x)[1]$. Mathematically that is:

$$
|f(x)| \leq M|g(x)| \text { for all } \quad x \geq x_{0}
$$

where $x_{0}$ is some large value that exists in the domain of $f$ and $g$. This can be extended to describe the behavior of $f$ near some real number $a$ :

$$
f(x)=\mathcal{O}[g(x)] \quad \text { as } \quad x \rightarrow a,
$$

if and only if there exist positive numbers $\delta$ and $M$ such that

$$
|f(x)| \leq M|g(x)| \text { for all }|x-a| \leq \delta
$$

Little-o notation, while similar to big-O, makes a stronger statement regarding the relationship between two functions $f$ and $g$. Informally, if we write $f(x)=o[g(x)]$ we are asserting that $g(x)$ grows much faster than $f(x)[1]$. Mathematically we define little-o as:

$$
f(x)=o[g(x)] \quad \text { as } \quad x \rightarrow \infty,
$$

if for every positive constant $\epsilon$ there exists a constant $N$ such that

$$
|f(x)| \leq \epsilon|g(x)| \quad \text { for all } \quad x \geq N
$$

Comparing Equations (1.2) and (1.3) to Equations (1.6) and (1.7) we see that big-O notation only requires that the condition in Equation (1.3) be met for at least one constant $M$. Conversely, little-o notation requires that the inequality in Equation (1.7) hold for every positive constant $\epsilon$, however small.

An example of an asymptotic approximation that arises in acoustics is the asymptotic form of the Hankel function. Hankel functions of the first and second kind arise as two linearly independent solutions to Bessel's equation, which specifies 
the behavior of the range solutions to the separated Helmholtz equation. In their closed form these functions are written:

$$
H_{0}^{(1)}(k r)=J_{0}(k r)+i Y_{0}(k r)
$$

and

$$
H_{0}^{(2)}(k r)=J_{0}(k r)-i Y_{0}(k r)
$$

where $J_{0}(k r)$ and $Y_{0}(k r)$ are Bessel functions of the first and second kind, respectively, corresponding to Bessel's equation of order 0. The Hankel functions in Equations (1.8) and (1.9) express outward- and inward-propagating cylindrical wave solutions to the cylindrical wave equation (or vice versa depending on the sign convention chosen for the time dependence of the acoustic source).

Hankel functions of the first and second kind each admit a contour integral representation. Namely:

$$
H_{\nu}^{(1)}(z)=\frac{1}{\pi} \int_{C_{1}} e^{-i z \sin \zeta+i \nu \zeta} d \zeta
$$

and

$$
H_{\nu}^{(2)}(z)=\frac{1}{\pi} \int_{C_{2}} e^{-i z \sin \zeta+i \nu \zeta} d \zeta
$$

where the contours $C_{1}$ and $C_{2}$ exist in the complex $\zeta$-plane. The contour $C_{1}$ traces a path from $A=-\pi+i \infty$ to $B=-i \infty$ while $C_{2}$ traces a path from $A=-i \infty$ to $B=\pi+i \infty$. Following reference [2], if we consider $\operatorname{Re}(z)>0$ one can apply the method of steepest descent to express the integrals in Equations (1.10) and (1.11) in the form of a series of inverse powers of $z$, where $z$ is taken to be large. This leads to asymptotic forms of the Hankel functions of the first and second kind. In the case of $H_{\nu}^{(1)}(z)$ we have

$$
H_{\nu}^{(1)}(z)=\mathcal{O}\left[\sqrt{\frac{2}{\pi z}} e^{i(z-\nu \pi / 2-(\pi / 4))}\right] \quad \text { for } \quad|z| \rightarrow \infty .
$$


If we now replace $z$ in Equation (1.12) with $k r$ and let $k r \rightarrow \infty$ and $\nu=0$ (corresponding to the zeroth order Bessel's equation) we arrive at an asymptotic form of the Hankel function in Equation (1.8) that is used frequently in acoustic propagation problems:

$$
H_{0}^{(1)}(k r) \sim \sqrt{\frac{2}{\pi k r}} e^{i(k r-(\pi / 4))} .
$$

Similarly, for Equation (1.9) we obtain

$$
H_{0}^{(2)}(k r) \sim \sqrt{\frac{2}{\pi k r}} e^{-i(k r-(\pi / 4))}
$$

Here $k=\omega / c$ is the acoustic wavenumber, $\omega$ is the angular frequency of an acoustic source, $c$ is the sound speed of the acoustic medium, and $r$ is range from the source. The asymptotic expressions in Equations (1.13) and (1.14) are often used to model the radiation of outgoing and incoming energy, respectively, in the normal modes solution to the acoustic wave equation to be discussed in detail in later sections.

\subsection{The Acoustic Wave Equation}

In mathematical physics, the acoustic wave equation is a second order partial differential equation (PDE) that governs the propagation of acoustic energy through a material medium. This equation describes the spatial and temporal evolution of acoustic pressure or particle velocity as a set of acoustic waves radiates through a particular environment. In underwater acoustics, the environment is specified through acoustic variables such as sound speed and density in water, as well as through boundary conditions at the surface and seafloor. Except for the case of very large wave amplitudes, a linear wave equation is used to describe acoustic processes underwater, which is a sufficient approximation for the problems of interest here. As such, the derivation of the linear acoustic wave equation will now be treated. 
Derivation of the linear acoustic wave equation involves manipulation of three basic hydrodynamic equations: conservation of mass, conservation of linear momentum, and the thermodynamic equation of state for a fluid. The first two of these three equations are derived by applying Reynold's Transport Theorem to mass and linear momentum, respectively, for an infinitesimally small control volume. Reynold's Transport Theorem for a control volume states that the time derivative of a particular fluid system property, $B$, equals the change of $B$ within the control volume plus the flux of $B$ out of the control surface, minus the flux of $B$ into the control surface [3]. Mathematically, we write:

$$
\frac{d}{d t}\left(B_{\text {syst }}\right)=\frac{d}{d t}\left(\int_{C V} \beta \rho \mathrm{d} V\right)+\int_{C S} \beta \rho(\boldsymbol{v} \bullet \boldsymbol{n}) \mathrm{d} A,
$$

where $\beta=d B / d m$ is the intensive value, or the amount of $B$ per unit mass in the fluid element, $\rho$ is density, typically measured in kilograms per cubic meters and $\boldsymbol{v}$ is the fluid particle velocity, a vector quantity measured in meters per second. $C V$ represents the control volume, while $C S$ represents the control surface enclosing the control volume, and $\boldsymbol{n}$ is the outward unit normal vector to the control surface.

In the case of conservation of mass, the fluid property of interest is the system mass, $m$. That is, $B=m$ and $\beta=d m / d m=1$ in Equation (1.15). Applying Reynold's Transport Theorem to this property for an infinitesimally small control volume and recognizing that the time derivative of the system mass is equal to zero (i.e. mass conservation) leads to the following hydrodynamic equation for mass, also known as the continuity equation:

$$
\frac{\partial \rho}{\partial t}+\nabla \cdot(\rho \boldsymbol{v})=0
$$

where $\boldsymbol{\nabla}$ is understood to be the gradient vector defined by

$$
\boldsymbol{\nabla}=\left\langle\frac{\partial}{\partial x}, \frac{\partial}{\partial y}, \frac{\partial}{\partial z}\right\rangle
$$


This equation requires no assumptions except that the density and velocity are continuum functions. That is, the flow may be steady or unsteady, viscous or inviscid, compressible or incompressible. However, the equation does not allow for any source or sink singularities within the fluid element [3].

For conservation of linear momentum, the fluid property of interest becomes the product of the system mass times its velocity. That is, $B=m \boldsymbol{v}$ and $\beta=d B / d m=\boldsymbol{v}$ in Equation (1.15). Recognizing again that the system mass is constant, the left side of Equation (1.15) becomes the product of mass and acceleration. From Newton's second law we recognize that this product is equal to the sum of all forces acting on the system; that is, the sum of all surface and body forces acting on the control volume at rest.

In the case of body forces, we include the gravitational force on the fluid element. The surface forces are more complicated and include the sum of hydrostatic pressure plus viscous stresses acting on the six fluid element faces. Unlike velocity, which is a three-component vector, the stresses and strain rates corresponding to the surface forces on the fluid element are nine-component tensors. Careful analysis shows that it is not the stresses themselves, but their gradients, that cause a net force on the differential control volume.

Substituting the sum of the body and surface forces into the left side of Reynold's Transport Theorem for linear momentum leads to the following general expression for the conservation of linear momentum in a differential control volume:

$$
\rho \boldsymbol{g}-\nabla p+\nabla \cdot \boldsymbol{\tau}_{i j}=\rho \frac{D \boldsymbol{v}}{D t}
$$

where

$$
\frac{D}{D t}\{\cdot\}=\frac{\partial}{\partial t}\{\cdot\}+\boldsymbol{v} \cdot \nabla\{\cdot\}
$$

is known as the material or total derivative, $\boldsymbol{g}$ is the gravity force vector, and $\boldsymbol{\tau}_{\boldsymbol{i} \boldsymbol{j}}$ is 
the viscous stress tensor acting on the fluid element. This expression holds for arbitrary body and surface forces and it states that: gravity force per unit volume+ pressure force per unit volume + viscous force per unit volume $=$ density $\times$ acceleration. If we assume frictionless flow and negligible gravitational force the conservation of linear momentum equation is given by:

$$
-\nabla p=\rho\left[\frac{\partial \boldsymbol{v}}{\partial t}+\boldsymbol{v} \cdot \boldsymbol{\nabla v}\right],
$$

where $p$ is pressure measured in Newtons per square meter, also referred to as pascals. This equation is known as Euler's equation and it is the typical form of the conservation of linear momentum that is used in the derivation of the acoustic wave equation.

Next we consider the state equation for a fluid. In physics and thermodynamics, an equation of state is a relation between state variables of a thermal system. These variables include but are not limited to pressure, density, temperature, entropy, and enthalpy. Typically, knowing the values of two of these state variables allows us to fix a third. That is, state relations typically express one thermodynamic variable as a function of two other thermodynamic variables.

In the case of an ideal gas, we have an explicit formula for the equation of state known as the ideal gas law. In the case of fluids, there is no comparable "ideal liquid law." Instead, the state equation for liquids typically involves an empirical relationship between pressure, density and a third thermodynamic variable. In underwater acoustic applications, this third variable is taken to be entropy, $S$, and the general equation of state for our fluid of interest is written as:

$$
p=p(\rho, S)
$$

Armed with Equations (1.16)-(1.21) we are now ready to begin derivation of the linear acoustic wave equation. This will be done following the notation of reference $[2]$. 
In the absence of a sound wave the undisturbed fluid variables of interest have the following values:

$$
\begin{aligned}
& \rho^{\prime}=\rho_{0} \\
& p^{\prime}=p_{0} \\
& \boldsymbol{v}^{\prime}=\boldsymbol{v}_{\mathbf{0}}=0 \\
& S^{\prime}=S_{0} \\
& Q^{\prime}=Q_{0}=0,
\end{aligned}
$$

where $Q^{\prime}$ represents the strength of our acoustic source. Note that $Q_{0}$ and $\boldsymbol{v}_{\mathbf{0}}$ are zero since the fluid is at rest (i.e. no acoustic wave is present). Now suppose that the acoustic source is turned on with source strength of $Q_{1}$. We assume this source to be a vibrating object whose amplitude and velocity of vibration are so small that they cause only a small perturbation of the undisturbed fluid variables in Equation (1.22). Let these perturbations be denoted by $\rho_{1}, p_{1}, \boldsymbol{v}_{\mathbf{1}}$, and $S_{1}$. Note that the source strength, $Q_{1}$, is of the same order of magnitude as the perturbed fluid variables. Given this information, the total density, pressure, velocity, entropy, and source strength can be represented by the expressions:

$$
\begin{aligned}
& \rho^{\prime}=\rho_{0}+\rho_{1} \\
& p^{\prime}=p_{0}+p_{1} \\
& \boldsymbol{v}^{\prime}=\boldsymbol{v}_{\mathbf{0}}+\boldsymbol{v}_{\mathbf{1}}=\boldsymbol{v}_{\mathbf{1}} \\
& S^{\prime}=S_{0}+S_{1} \\
& Q^{\prime}=Q_{0}+Q_{1}=Q_{1} .
\end{aligned}
$$

Substituting the acoustic variables in Equation (1.23) into Equation (1.16) and (1.20) and assuming that squares or higher order terms involving the (small) perturbations of the acoustic variables can be neglected leads to linearized forms of the conservation of mass and conservation of linear momentum equations. Linearizing the state equation requires a bit more work. We expand the state equation 
about the equilibrium state of the fluid, again neglecting second and higher order terms in the acoustic variables. This leads to the following linearized equations:

$$
\begin{gathered}
\frac{\partial \rho_{1}}{\partial t}+\boldsymbol{v}_{\mathbf{1}} \cdot \boldsymbol{\nabla} \rho_{0}+\rho_{0} \boldsymbol{\nabla} \cdot \boldsymbol{v}_{\mathbf{1}}=Q_{1} \\
\frac{\partial \boldsymbol{v}_{\mathbf{1}}}{\partial t}=-\frac{1}{\rho_{0}} \boldsymbol{\nabla} p_{1} \\
p_{1}=\rho_{1} c^{2} .
\end{gathered}
$$

To derive a linear wave equation we first take the divergence of the momentum equation in Equation (1.25), leading to the expression:

$$
\frac{\partial}{\partial t} \boldsymbol{\nabla} \cdot \boldsymbol{v}_{\mathbf{1}}+\boldsymbol{\nabla} \cdot\left(\frac{1}{\rho_{0}} \boldsymbol{\nabla} p_{1}\right)=0 .
$$

Next, we recall the constitutive equation for isentropic flow,

$$
\frac{\mathrm{D} p^{\prime}}{\mathrm{D} t}=c^{2} \frac{\mathrm{D} \rho^{\prime}}{\mathrm{D} t}
$$

where $c$ is the speed of sound in our fluid of interest. We simplify this equation by substituting the perturbed pressure, $p^{\prime}$, and the perturbed density, $\rho^{\prime}$, from Equation (1.23) into the equation and again assume that squares or higher order terms involving the perturbations of the acoustic variables can be neglected. Doing so, we arrive at:

$$
\frac{\partial p_{1}}{\partial t}+\boldsymbol{v}_{\mathbf{1}} \cdot \boldsymbol{\nabla} p_{0}=c^{2}\left[\frac{\partial \rho_{1}}{\partial t}+\boldsymbol{v}_{\mathbf{1}} \bullet \boldsymbol{\nabla} \rho_{0}\right]
$$

We now combine Equation (1.29) with Equation (1.24) to obtain the expression:

$$
\frac{1}{c^{2}}\left[\frac{\partial p_{1}}{\partial t}+\boldsymbol{v}_{\mathbf{1}} \cdot \boldsymbol{\nabla} p_{0}\right]+\rho_{0} \boldsymbol{\nabla} \cdot \boldsymbol{v}_{\mathbf{1}}=Q_{1}
$$

From thermodynamic considerations one has that $\nabla p_{0} / p_{0} \ll \nabla T_{0} / T_{0}$, where $T_{0}$ corresponds to the ambient temperature of the fluid medium. As such, we have that the $\boldsymbol{\nabla} p_{0}$ term is negligible, leading to the following:

$$
\frac{1}{c^{2}} \frac{\partial p_{1}}{\partial t}+\rho_{0} \boldsymbol{\nabla} \cdot \boldsymbol{v}_{\mathbf{1}} \approx Q_{1}
$$


We now differentiate Equation (1.31) with respect to time and use Equation (1.27) to eliminate $\boldsymbol{\nabla} \bullet \boldsymbol{v}_{\mathbf{1}}$, arriving at:

$$
\frac{1}{c^{2}} \frac{\partial^{2} p_{1}}{\partial t^{2}}-\rho_{0} \boldsymbol{\nabla} \cdot\left(\frac{1}{\rho_{0}} \nabla p_{1}\right) \approx \frac{\partial Q_{1}}{\partial t}
$$

Going forward, subscripts on the acoustic variables will be dropped and it is understood that all variables refer to their perturbative quantities. In the case of density, we will consider the variation due to the acoustic disturbance to be negligible and $\rho$ will refer to the density of the medium at rest. Taking this into account and rearranging terms in Equation (1.32), the following expression for the linear acoustic wave equation is obtained:

$$
\rho \boldsymbol{\nabla} \cdot\left(\frac{1}{\rho} \nabla p\right)-\frac{1}{c^{2}} \frac{\partial^{2} p}{\partial t^{2}}=-\frac{\partial Q}{\partial t}
$$

If we consider density to be constant in space and the source strength to be time independent we arrive at the standard form of the linear homogeneous wave equation for small-amplitude acoustic processes:

$$
\nabla^{2} p-\frac{1}{c^{2}} \frac{\partial^{2} p}{\partial t^{2}}=0
$$

where $\nabla^{2}$ is the Laplacian operator:

$$
\nabla^{2}\{\cdot\}=\frac{\partial^{2}}{\partial x^{2}}\{\cdot\}+\frac{\partial^{2}}{\partial y^{2}}\{\cdot\}+\frac{\partial^{2}}{\partial z^{2}}\{\cdot\} .
$$

\subsection{The Acoustic Velocity Potential}

Following a procedure similar to that used in deriving the wave equation for pressure, an acoustic wave equation for particle velocity may be obtained. To accomplish this, we will assume that we are a significant distance from the source and that $\nabla \rho_{0} / \rho_{0} \ll 1$. Doing so, Equation (1.24) becomes:

$$
\frac{\partial \rho_{1}}{\partial t}=-\rho_{0} \boldsymbol{\nabla} \cdot \boldsymbol{v}_{\mathbf{1}}
$$


We now take the gradient of Equation (1.36) and the time derivative of Equation (1.25). Combining the two results using Equation (1.26) and dropping subscripts we arrive at a wave equation for particle velocity. This equation is:

$$
\frac{1}{\rho} \boldsymbol{\nabla}\left(\rho c^{2} \boldsymbol{\nabla} \cdot \boldsymbol{v}\right)-\frac{\partial^{2} \boldsymbol{v}}{\partial t^{2}}=\mathbf{0}
$$

where $\boldsymbol{v}$ is a function of the position vector $\boldsymbol{r}$ and time $t$. If the density of the acoustic medium is slowly varying, the vector equation in Equation (1.37) may be transformed into a scalar equation by defining a velocity potential $\Phi(\boldsymbol{r}, t)$ given by

$$
\boldsymbol{v}(\boldsymbol{r}, t)=\nabla \Phi(\boldsymbol{r}, t)
$$

If one substitutes Equation (1.38) into Equation (1.37) and assumes that $\boldsymbol{\nabla} \rho=\mathbf{0}$, then the following expression is obtained

$$
\boldsymbol{\nabla}\left(c^{2} \nabla^{2} \Phi-\frac{\partial^{2} \Phi}{\partial t^{2}}\right)=\mathbf{0}
$$

This expression is satisfied if the velocity potential satisfies the scalar wave equation

$$
\nabla^{2} \Phi-\frac{1}{c^{2}} \frac{\partial^{2} \Phi}{\partial t^{2}}=0
$$

which we note is identical to that for pressure in Equation (1.34).

A direct relationship between velocity potential and pressure may be obtained through the introduction of a displacement potential, $\psi$, defined by

$$
\boldsymbol{d}(\boldsymbol{r}, t)=\boldsymbol{\nabla} \psi(\boldsymbol{r}, t)
$$

where $\boldsymbol{d}(\boldsymbol{r}, t)$ is the particle displacement vector given by the kinematic relationship $\boldsymbol{v}=\partial \boldsymbol{d} / \partial t$. Combining Equations (1.36), (1.26), and (1.41), we arrive at the relationship between pressure and the displacement potential

$$
p=-K \nabla^{2} \psi
$$


where we recognize that $K$ is the bulk modulus defined by

$$
K=\rho c^{2} .
$$

Equation (1.42) is the equivalent of Hooke's law for an ideal, linearly elastic fluid. Note, that the displacement potential is governed by a wave equation identical to that of pressure and the velocity potential:

$$
\nabla^{2} \psi-\frac{1}{c^{2}} \frac{\partial^{2} \psi}{\partial t^{2}}=0
$$

Combining Equations (1.42)-(1.44) results in the following alternative expression for the acoustic pressure

$$
p=-\rho \frac{\partial^{2} \psi}{\partial t^{2}}
$$

Next, we assume a continuous wave time harmonic acoustic source, leading to a time dependence of the form $e^{-i \omega t}$, where $\omega=2 \pi f$ is angular frequency of the source in radians per second and $f$ is frequency in Hertz. Such a source may be represented by the expression:

$$
Q(\boldsymbol{r}, t)=\hat{Q}(\boldsymbol{r}, \omega) e^{-i \omega t}
$$

This leads to the following expression for the acoustic pressure:

$$
p(\boldsymbol{r}, t)=\hat{p}(\boldsymbol{r}, \omega) e^{-i \omega t}
$$

An expression for the acoustic velocity potential is similarly obtained. This is:

$$
\Phi(\boldsymbol{r}, t)=\hat{\Phi}(\boldsymbol{r}, \omega) e^{-i \omega t}
$$

One can show that $\Phi=\partial \psi / \partial t$. Using this relationship, we finally arrive at the following relationship between spatially-dependent pressure and velocity potential for a time harmonic acoustic source:

$$
\hat{p}(\boldsymbol{r}, \omega)=i \omega \rho \hat{\Phi}(\boldsymbol{r}, \omega)
$$


The relationship in Equation (1.49) will be utilized a number of times throughout the remainder of the presented work. While the proposed coupled-mode theory is developed in the context of velocity potentials, there will be times at which converting quantities of interest to pressure provides for a more intuitive discussion.

\subsection{The Helmholtz Equation}

Time-independence of the coefficients to the differential operators in Equation (1.33) allows for the acoustic wave equation to be further simplified. This is accomplished through application of the following Fourier transform operator:

$$
\mathcal{F}[f(t)]=\hat{f}(\omega)=\int_{-\infty}^{\infty} f(t) e^{i \omega t} \mathrm{~d} t
$$

The inverse of which is:

$$
\mathcal{F}^{-1}[\hat{f}(\omega)]=f(t)=\frac{1}{2 \pi} \int_{-\infty}^{\infty} \hat{f}(\omega) e^{-i \omega t} \mathrm{~d} \omega
$$

Application of the transform in Equation (1.50) to Equation (1.33) leads to what is known as the Helmholtz equation or reduced wave equation, expressed as:

$$
\rho \boldsymbol{\nabla} \bullet\left(\frac{1}{\rho} \nabla \hat{p}(\boldsymbol{r}, \omega)\right)+k^{2}(\boldsymbol{r}) \hat{p}(\boldsymbol{r}, \omega)=i \omega \hat{Q}(\boldsymbol{r}, \omega),
$$

where $k(\boldsymbol{r})$ is the medium wavenumber at radial frequency $\omega$ and sound velocity $c(\boldsymbol{r})$, that is:

$$
k(\boldsymbol{r})=\frac{\omega}{c(\boldsymbol{r})}
$$

Recall that for a time harmonic acoustic source of a single frequency the timedependent pressure may be written as in Equation (1.47). Under these conditions, Equation (1.52) is equivalently obtained by substituting the expressions on the right sides of Equations (1.46) and (1.47) into the wave equation in Equation (1.33) and carrying out the differentiation with respect to time.

A Helmholtz equation similar to Equation (1.52) may be obtained for the velocity potential by utilizing the relationship given in Equation (1.49). Doing so 
leads to the result:

$$
\boldsymbol{\nabla} \cdot\left(\frac{1}{\rho} \nabla \rho \hat{\Phi}(\boldsymbol{r}, \omega)\right)+k^{2}(\boldsymbol{r}) \hat{\Phi}(\boldsymbol{r}, \omega)=\frac{\hat{Q}(\boldsymbol{r}, \omega)}{\rho}
$$

Note that if one assumes constant density, the left sides of Equations (1.52) and (1.54) are identical.

Due to the reduction in dimension, the PDE's in Equations (1.52) and (1.54) are simpler to solve than the full wave equation. This simplification is achieved, however, at the cost of having to evaluate the inverse Fourier transform given by Equation (1.51) to achieve the final solution.

\subsection{Source Representation}

Underwater sound due to natural or artificial phenomena is produced through forced mass injection [4]. For acoustic sources with complex geometries, mathematically representing this mass injection term can be difficult. In the case of an omni-directional point source, an analytical expression for the forcing term may be derived by recognizing that the acoustic field is produced by a small sphere inducing body forces on the fluid medium through expansions and contractions. These body forces create small particle displacements in the fluid that propagate out from the source as spherical acoustic waves.

If we consider the medium to be infinite (i.e. unbounded), then the acoustic field will be spherically symmetric and we need only concern ourselves with the radial distance from the center of the source, $r$. Given this set of assumptions, the particle displacement field in Equation (1.41) may be written as

$$
d(r, t)=\frac{\partial \psi(r, t)}{\partial r}
$$

where $\psi(r, t)$ is again recognized as the displacement potential function. If we then define the radius of the sphere with the symbol $a$ and apply a no-slip boundary 
condition at the source-fluid interface we recognize that

$$
d(a, t)=D(t)
$$

where $D(t)$ is surface displacement of the sphere at time $t$ with dimensions of length.

To simplify the discussion we will consider the density in the acoustic medium to be constant. Taking this into account, the Fourier-transformed displacement potential for our propagation scenario of interest satisfies the Helmholtz equation

$$
\left[\frac{1}{r^{2}} \frac{\partial}{\partial r} r^{2} \frac{\partial}{\partial r}+k^{2}\right] \hat{\psi}(r, \omega)=\hat{S}_{\psi}(\omega) \hat{Q}(r)
$$

with boundary condition

$$
\hat{d}(a, \omega)=\hat{D}(\omega)
$$

Here $\hat{Q}(r)$ is representative of our point source and $\hat{S}_{\psi}(\omega)$ is a source strength term to be analytically derived. Note that the field for this scenario depends solely on range from the source. As such, Equation (1.57) has been written in spherical coordinates for convenience.

Considering ranges a significant distance from the source (i.e. $r \gg 0$ ) the displacement potential satisfies the homogeneous equivalent of Equation (1.57), the solution of which is a linear combination of incoming and outgoing waves given by the expressions:

$$
\hat{\psi}(r, \omega)= \begin{cases}(A / r) e^{i k r} & \text { outgoing wave } \\ (B / r) e^{-i k r} & \text { incoming wave. }\end{cases}
$$

Since the sphere is assumed to be the only source in the infinite medium there are no incoming waves thus requiring that $B=0$. Thus for the current case of interest

$$
\hat{\psi}(r, \omega)=A \frac{e^{i k r}}{r}
$$


with corresponding displacement field

$$
\hat{d}(r, \omega)=A e^{i k r}\left(\frac{i k}{r}-\frac{1}{r^{2}}\right)
$$

where Equation (1.61) was obtained by applying the Fourier transform operator in Equation (1.50) to the displacement field in Equation (1.55) and carrying out the differentiation with respect range, $r$. Our assumed model of a simple point source requires that the radius of the sphere be small compared to the acoustic wavelength. This necessitates that $k a \ll 1$, in which case Equation (1.61) takes the form

$$
\hat{d}(a, \omega)=A e^{i k a} \frac{i k a-1}{a^{2}} \approx-\frac{A}{a^{2}} .
$$

Utilizing Equation (1.58), we then arrive at the following solution for the wave amplitude $A$ :

$$
A=-a^{2} \hat{D}(\omega)
$$

If we now define the source strength $\hat{S}_{\psi}(\omega)=4 \pi a^{2} \hat{D}(\omega)$ as the volumeinjection amplitude produced by the source at frequency $\omega$, the following solution is obtained for the field in the fluid,

$$
\hat{\psi}(r, \omega)=-\hat{S}_{\psi}(\omega) \frac{e^{i k r}}{4 \pi r}
$$

where the source strength $\hat{S}_{\psi}(\omega)$ has dimensions of cubic length, or volume.

The ratio appearing in Equations (1.64) is known as the free-space Green's function, which has general form

$$
g_{\omega}\left(\boldsymbol{r}, \boldsymbol{r}_{0}\right)=\frac{e^{i k\left|\boldsymbol{r}-\boldsymbol{r}_{0}\right|}}{4 \pi\left|\boldsymbol{r}-\boldsymbol{r}_{0}\right|}
$$

where $\boldsymbol{r}_{0}$ is the location of the source. The free-space Green's function satisfies the following inhomogeneous Helmholtz equation,

$$
\left[\nabla^{2}+k^{2}(\boldsymbol{r})\right] g_{\omega}\left(\boldsymbol{r}, \boldsymbol{r}_{0}\right)=-\delta\left(\boldsymbol{r}-\boldsymbol{r}_{0}\right)
$$


which can be verified by integrating Equation (1.66) over a small volume containing the source point. Taking this into account, if one sets $\hat{S}_{\psi}(\omega)$ to unity in Equation (1.57) it is easily verified that $\hat{Q}(r)=-\delta(r)$, or in general

$$
\hat{Q}(\boldsymbol{r})=-\delta\left(\boldsymbol{r}-\boldsymbol{r}_{0}\right)
$$

A source strength for velocity potential, $\hat{S}_{\Phi}(\omega)$, may be obtained by recalling that $\Phi=\partial \psi / \partial t$. Applying the Fourier transform operator in Equation (1.50) to this relationship and taking into account Equation (1.64), we find that

$$
\hat{S}_{\Phi}(\omega)=-i \omega \hat{S}_{\psi}(\omega)=-i \omega 4 \pi a^{2} \hat{D}(\omega)
$$

where $\hat{S}_{\Phi}(\omega)$ has dimensions of cubic length over time. This implies that for the wave equation

$$
\left[\nabla^{2}+k^{2}\right] \hat{\Phi}(r, \omega)=\hat{S}_{\Phi}(\omega) \hat{Q}(r)
$$

the following is a solution:

$$
\hat{\Phi}(r, \omega)=-\hat{S}_{\Phi}(\omega) \frac{e^{i k r}}{4 \pi r}
$$

A source strength for acoustic pressure, $\hat{S}_{p}(\omega)$, may be obtained in a similar fashion by recalling the relationship in Equation (1.49). This leads to the definition:

$$
\hat{S}_{p}(\omega)=\rho \omega^{2} \hat{S}_{\psi}(\omega)=\rho \omega^{2} 4 \pi a^{2} \hat{D}(\omega)
$$

where $\hat{S}_{p}(\omega)$ has dimensions mass over squared time. This implies that for the wave equation

$$
\left[\nabla^{2}+k^{2}\right] \hat{p}(r, \omega)=\hat{S}_{p}(\omega) \hat{Q}(r)
$$

the following is a solution:

$$
\hat{p}(r, \omega)=-\hat{S}_{p}(\omega) \frac{e^{i k r}}{4 \pi r}
$$


Recalling Equations (1.52) and (1.54), we can redefine $\hat{S}_{p}(\omega)$ and $\hat{S}_{\Phi}(\omega)$ as:

$$
\hat{S}_{p}(\omega)=i \omega \dot{m}
$$

and

$$
\hat{S}_{\Phi}(\omega)=\frac{1}{\rho} \dot{m}
$$

where

$$
\dot{m}=-i \rho \omega 4 \pi a^{2} \hat{D}(\omega)
$$

Given these definitions, we see that for a point source in a medium with nonconstant density the Fourier-transformed complex pressure, $\hat{p}(\boldsymbol{r}, \omega)$, satisfies the equation:

$$
\rho \boldsymbol{\nabla} \cdot\left(\frac{1}{\rho} \nabla \hat{p}(\boldsymbol{r}, \omega)\right)+k^{2}(\boldsymbol{r}) \hat{p}(\boldsymbol{r}, \omega)=\hat{S}_{p}(\omega) \hat{Q}(\boldsymbol{r}) .
$$

Similarly, the Fourier-transformed velocity potential, $\hat{\Phi}(\boldsymbol{r}, \omega)$, satisfies

$$
\boldsymbol{\nabla} \cdot\left(\frac{1}{\rho} \nabla \rho \hat{\Phi}(\boldsymbol{r}, \omega)\right)+k^{2}(\boldsymbol{r}) \hat{\Phi}(\boldsymbol{r}, \omega)=\hat{S}_{\Phi}(\omega) \hat{Q}(\boldsymbol{r}) .
$$

Note that $\dot{m}$ in Equations (1.74) and (1.75) has dimensions of mass over time. As such, this term represents the amount of mass per unit time being injected into the acoustic medium by an omni-directional point source. Taking this into account, a source is said to have "unit strength" when $\dot{m}=1$, meaning that the source injects one unit of mass per unit of time into the acoustic medium. In SI units this

corresponds to $1 \mathrm{~kg} \mathrm{~s}^{-1}$ of mass injection. Under these conditions $\hat{S}_{p}(\omega)=i \omega$ and $\hat{S}_{\Phi}(\omega)=1 / \rho$, as in Equations (1.52) and (1.54).

\subsection{General Solution: Green's Function}

The application of Green's functions also allows for the development of a solution to the general problem of a bounded medium with general source. To solve this problem we consider the wave equation:

$$
\left[\nabla^{2}+k^{2}(\boldsymbol{r})\right] \hat{\Phi}(\boldsymbol{r}, \omega)=\hat{F}(\boldsymbol{r}, \omega)
$$


where the acoustic medium occupies a volume $V$ bounded by a surface $S$, with prescribed conditions at the boundary. Here $\hat{F}(\boldsymbol{r}, \omega)$ is a general forcing term, which includes the source strength and has dimensions of inverse seconds. Next, we formulate a general solution to Equation (1.66) as a sum of the particular solution, $g_{\omega}\left(\boldsymbol{r}, \boldsymbol{r}_{0}\right)$, and a homogeneous solution $h_{\omega}\left(\boldsymbol{r}, \boldsymbol{r}_{0}\right)$, that is,

$$
G_{\omega}\left(\boldsymbol{r}, \boldsymbol{r}_{0}\right)=g_{\omega}\left(\boldsymbol{r}, \boldsymbol{r}_{0}\right)+h_{\omega}\left(\boldsymbol{r}, \boldsymbol{r}_{0}\right)
$$

Recognizing that by definition,

$$
\left[\nabla^{2}+k^{2}(\boldsymbol{r})\right] h_{\omega}\left(\boldsymbol{r}, \boldsymbol{r}_{0}\right)=0
$$

we find that

$$
\left[\nabla^{2}+k^{2}(\boldsymbol{r})\right] G_{\omega}\left(\boldsymbol{r}, \boldsymbol{r}_{0}\right)=-\delta\left(\boldsymbol{r}-\boldsymbol{r}_{0}\right)
$$

Following a process outlined in reference [5], we now multiply Equation (1.79) by $G_{\omega}\left(\boldsymbol{r}, \boldsymbol{r}_{0}\right)$ and multiply Equation $(1.82)$ by $\hat{\Phi}(\boldsymbol{r}, \omega)$. Subtracting the two expressions, interchanging $\boldsymbol{r}$ with $\boldsymbol{r}_{0}$, and integrating over volume we obtain

$$
\begin{aligned}
& \int_{V}\left[G_{\omega}\left(\boldsymbol{r}, \boldsymbol{r}_{0}\right) \nabla_{0}^{2} \hat{\Phi}\left(\boldsymbol{r}_{0}, \omega\right)-\hat{\Phi}\left(\boldsymbol{r}_{0}, \omega\right) \nabla_{0}^{2} G_{\omega}\left(\boldsymbol{r}, \boldsymbol{r}_{0}\right)\right] \mathrm{d} V_{0} \\
= & \int_{V} \hat{\Phi}\left(\boldsymbol{r}_{0}, \omega\right) \delta\left(\boldsymbol{r}-\boldsymbol{r}_{0}\right) \mathrm{d} V_{0}+\int_{V} \hat{F}\left(\boldsymbol{r}_{0}, \omega\right) G_{\omega}\left(\boldsymbol{r}, \boldsymbol{r}_{0}\right) \mathrm{d} V_{0},
\end{aligned}
$$

where we have used the fact that the Green's function is symmetric, i.e., $G_{\omega}\left(\boldsymbol{r}, \boldsymbol{r}_{0}\right)=G_{\omega}\left(\boldsymbol{r}_{0}, \boldsymbol{r}\right)$. Application of Green's theorem to the term on the left side of Equation (1.83) and the sifting property of the Dirac delta function to the right side of Equation (1.83) leads to

$$
\begin{aligned}
\hat{\Phi}(\boldsymbol{r}, \omega)=\int_{S}\left[G_{\omega}\left(\boldsymbol{r}, \boldsymbol{r}_{0}\right) \frac{\partial \hat{\Phi}\left(\boldsymbol{r}_{0}, \omega\right)}{\partial \boldsymbol{n}_{0}}\right. & \left.-\hat{\Phi}\left(\boldsymbol{r}_{0}, \omega\right) \frac{\partial G_{\omega}\left(\boldsymbol{r}, \boldsymbol{r}_{0}\right)}{\partial \boldsymbol{n}_{0}}\right] \mathrm{d} S_{0} \\
& -\int_{V} \hat{F}\left(\boldsymbol{r}_{0}, \omega\right) G_{\omega}\left(\boldsymbol{r}, \boldsymbol{r}_{0}\right) \mathrm{d} V_{0},
\end{aligned}
$$

where $\boldsymbol{n}_{0}$ is the outward-pointing normal on the surface $S$. Equation (1.84) is the general solution to Equation (1.79) for a bounded acoustic medium with general 
forcing term. Usefulness of Equation (1.84), however, depends on the ability to solve the integral equation, which may be nontrivial.

\section{List of References}

[1] N. G. de Brujin, Asymptotic Methods in Analysis. New York: Dover Publications, 1981.

[2] C. A. Boyles, Acoustic Waveguides: Applications to Oceanic Science. New York: Wiley-Interscience, 1984.

[3] F. M. White, Fluid Mechanics, 6th ed. New York: McGraw-Hill, 2006.

[4] F. B. Jensen, W. A. Kuperman, M. B. Porter, and H. Schmidt, Computational Ocean Acoustics, 1st ed. New York: Springer-Verlag, 2000.

[5] G. V. Frisk, Ocean and Seabed Acoustics: A Theory of Wave Propagation. New Jersey: Prentice-Hall, 1994. 


\section{CHAPTER 2}

\section{Normal Mode Theory}

\subsection{Background}

The method of normal modes provides a means of solving the wave equation that does not require computation of a volume integral. This method involves solving a depth-dependent equation that results in a decomposition of the field into a set of modes of vibration, roughly similar to that of a vibrating string. The complete acoustic field is constructed via a weighted sum of these modes, where the weights are informed by the value of the modes at the depth of the sound source. In this section two approaches leading to the normal modes solution are presented. The first is based on a generalized application of the method of separation of variables, the derivation of which will be discussed in detail. The second approach, which involves the computation of a wavenumber contour integral via a sum of residues, will be discussed more generally as a means of connecting the normal modes method to the field of geometric acoustics.

\subsection{Solution via Generalized Separation of Variables}

We begin by recalling from Equation (1.77) that the Fourier-transformed complex pressure, $\hat{p}(\boldsymbol{r}, \omega)$, satisfies the Helmholtz wave equation:

$$
\rho \boldsymbol{\nabla} \cdot\left(\frac{1}{\rho} \boldsymbol{\nabla} \hat{p}(\boldsymbol{r}, \omega)\right)+k^{2}(\boldsymbol{r}) \hat{p}(\boldsymbol{r}, \omega)=\hat{S}_{p}(\omega) \hat{Q}(\boldsymbol{r})
$$

where $\hat{S}_{p}(\omega)=i \omega \dot{m}$ and $\dot{m}=-i \rho \omega 4 \pi a^{2} \hat{D}(\omega)$. If we now restrict ourselves to the case of a cylindrical waveguide with azimuthal symmetry, Equation (2.1) takes the form:

$$
\frac{1}{r} \frac{\partial}{\partial r}\left(r \frac{\partial \hat{p}}{\partial r}\right)+\rho(z) \frac{\partial}{\partial z}\left(\frac{1}{\rho(z)} \frac{\partial \hat{p}}{\partial z}\right)+k^{2} \hat{p}=-i \omega \dot{m} \frac{\delta(r) \delta\left(z-z_{s}\right)}{2 \pi r}
$$


where $z_{s}$ corresponds to the depth of the source. Here we have expanded the first term on the left side of Equation (2.1) and recalled the definition of the Laplacian operator in cylindrical coordinates:

$$
\nabla^{2}\{\cdot\}=\frac{1}{r} \frac{\partial}{\partial r}\left(r \frac{\partial}{\partial r}\{\cdot\}\right)+\frac{1}{r^{2}} \frac{\partial^{2}}{\partial \theta^{2}}\{\cdot\}+\frac{\partial^{2}}{\partial z^{2}}\{\cdot\}
$$

Note that our assumption of azimuthal symmetry dictates that the derivative involving the angular term, $\theta$, in Equation (2.3) vanishes.

To investigate the solution to Equation (2.2), we apply the method of separation of variables to its associated homogeneous (unforced) equation. In doing so, we seek a solution of the form $\hat{p}(r, z)=\varphi(r) u(z)$. We substitute this proposed solution into the homogeneous differential equation and divide by $\varphi(r) u(z)$, leading to the expression:

$$
\frac{1}{\varphi}\left[\frac{1}{r} \frac{d}{d r}\left(r \frac{d \varphi}{d r}\right)\right]+\frac{1}{u}\left[\rho(z) \frac{d}{d z}\left(\frac{1}{\rho(z)} \frac{d u}{d z}\right)+\frac{\omega^{2}}{c^{2}(z)} u\right]=0 .
$$

We see that the first and second terms in brackets in this expression are solely functions of $r$ and $z$, respectively. The only way that this equation can be satisfied is if both terms are equal to a separation constant that we will denote by $k_{r}^{2}$. That is:

$$
\frac{1}{u}\left[\rho(z) \frac{d}{d z}\left(\frac{1}{\rho(z)} \frac{d u}{d z}\right)+\frac{\omega^{2}}{c^{2}(z)} u\right]=-\frac{1}{\varphi}\left[\frac{1}{r} \frac{d}{d r}\left(r \frac{d \varphi}{d r}\right)\right]=k_{r}^{2} .
$$

Concerning ourselves with the depth equation in Equation (2.5), bringing the separation constant over to the left side of the equation, and multiplying by $u$, we arrive at an ordinary differential equation for the depth solutions, $u(z)$ :

$$
\rho(z) \frac{d}{d z}\left[\frac{1}{\rho(z)} \frac{d u(z)}{d z}\right]+\left[\frac{\omega^{2}}{c^{2}(z)}-k_{r}^{2}\right] u(z)=0
$$

Assuming a pressure-release surface located at $z=0$ and a perfectly rigid bottom located at $z=H$ imposes the following boundary conditions on the solutions to 
Equation (2.6):

$$
\begin{aligned}
u(0) & =0 \\
\left.\frac{d u(z)}{d z}\right|_{z=H} & =0 .
\end{aligned}
$$

Equation (2.6) combined with the boundary conditions in Equation (2.7) define a classical Sturm-Liouville eigenvalue problem. There are an infinite number of solutions $u_{m}(z)$ to Equation (2.6) and we refer to these solutions as the eigenfunctions of the problem.

If we represent the differential operator in Equation (2.6) by $L$, then for each eigenfunction $u_{m}(z)$ there exists a corresponding eigenvalue $k_{r m}^{2}$ such that:

$$
L\left[u_{m}(z)\right]=k_{r m}^{2} u_{m}(z)
$$

Note also that the eigenfunctions or modes, $u_{m}(z)$, have the property of orthogonality, which is stated mathematically as:

$$
\int_{0}^{H} \rho^{-1}(z) u_{m}(z) u_{n}(z) \mathrm{d} z=\delta_{n m}
$$

Moreover, the eigenfunctions form a complete set thus allowing us to represent an arbitrary function as a sum of the eigenfunctions or normal modes. This allows us to further specify the form of our proposed solution to the original wave equation. Namely, we have:

$$
\hat{p}(r, z)=\sum_{m=1}^{\infty} \varphi_{m}(r) u_{m}(z),
$$

where we have expanded $\hat{p}(r, z)$ in the normal mode basis $u_{m}(z)$, and $\varphi_{m}(r)$ are our expansion coefficients, which we propose to be strictly a function of range, $r$.

If we substitute Equation (2.10) into Equation (2.2) and apply the property in Equation (2.8) we obtain the intermediate equation:

$$
\sum_{m=1}^{\infty}\left[\frac{1}{r} \frac{d}{d r}\left(r \frac{d \varphi_{m}(r)}{d r}\right) u_{m}(z)+k_{r m}^{2} \varphi_{m}(r) u_{m}(z)\right]=-i \omega \dot{m} \frac{\delta(r) \delta\left(z-z_{s}\right)}{2 \pi r}
$$


Taking into account the property in Equation (2.9), we now multiply Equation (2.11) by $\rho^{-1}(z) u_{n}(z)$ and integrate from 0 to $H$ resulting in the following rangeseparated equation:

$$
\frac{1}{r} \frac{d}{d r}\left(r \frac{d \varphi_{n}(r)}{d r}\right)+k_{r n}^{2} \varphi_{n}(r)=-i \omega \dot{m} \frac{\delta(r) u_{n}\left(z_{s}\right)}{2 \pi r \rho\left(z_{s}\right)} .
$$

Equation (2.12) is a standard equation known as Bessel's equation. To solve this equation, we define the Hankel transform:

$$
\mathcal{H}[f(r)]=F(\kappa)=\int_{0}^{\infty} f(r) J_{0}(\kappa r) r \mathrm{~d} r
$$

the inverse of which is

$$
\mathcal{H}^{-1}[F(\kappa)]=f(r)=\int_{0}^{\infty} F(\kappa) J_{0}(\kappa r) \kappa \mathrm{d} \kappa .
$$

Next we note the following property of the transform in Equation (2.13):

$$
\mathcal{H}\left[\frac{1}{r} \frac{d}{d r}\left(r \frac{d f(r)}{d r}\right)\right]=-\kappa^{2} F(\kappa) .
$$

Applying the operator in Equation (2.13) to Equation (2.12) and letting $F(\kappa)=\mathcal{H}\left[\varphi_{n}\right]$, we arrive at

$$
\left[-\kappa^{2}+k_{r n}^{2}\right] F(\kappa)=-\frac{i \omega \dot{m} u_{n}\left(z_{s}\right)}{2 \pi \rho\left(z_{s}\right)} \int_{0}^{\infty} \delta(r) J_{0}(\kappa r) \mathrm{d} r=-\frac{i \omega \dot{m} u_{n}\left(z_{s}\right)}{2 \pi \rho\left(z_{s}\right)}
$$

Solving for $F(\kappa)$, we have

$$
F(\kappa)=\frac{i \omega \dot{m} u_{n}\left(z_{s}\right)}{2 \pi \rho\left(z_{s}\right)\left[\kappa^{2}-k_{r n}^{2}\right]} .
$$

Next we apply the inverse Hankel transform in Equation (2.14) to $F(\kappa)$ in Equation (2.17) and note the identity

$$
K_{0}(\mu r)=\int_{0}^{\infty} \frac{J_{0}(\kappa r)}{\kappa^{2}+\mu^{2}} \kappa \mathrm{d} \kappa,
$$

where $K_{0}$ is the modified Bessel function of the second kind [1]. Letting $\mu=i k_{r n}$ in Equation (2.18) leads to an intermediate solution to Equation (2.12):

$$
\varphi_{n}(r)=\frac{i \omega \dot{m} u_{n}\left(z_{s}\right)}{2 \pi \rho\left(z_{s}\right)} K_{0}\left(i k_{r n} r\right) .
$$


Finally, we note that

$$
K_{0}(\zeta)=\left\{\begin{array}{cc}
\frac{1}{2} \pi i H_{0}^{(1)}(i \zeta), & -\pi<\arg \zeta \leq \frac{\pi}{2} \\
-\frac{1}{2} \pi i H_{0}^{(2)}(-i \zeta), & -\frac{\pi}{2}<\arg \zeta \leq \pi
\end{array}\right.
$$

For outgoing radiation we choose $H_{0}^{(1)}$ leading to the following solution to the range-separated equation in Equation (2.12):

$$
\varphi_{n}(r)=-\frac{\omega \dot{m} u_{n}\left(z_{s}\right)}{4 \rho\left(z_{s}\right)} H_{0}^{(1)}\left(k_{r n} r\right)
$$

Substituting the right side of Equation (2.21) into Equation (2.10) we arrive at the normal modes solution to Equation(2.2) for a point source in a cylindrical waveguide with azimuthal symmetry:

$$
\hat{p}(r, z)=-\frac{\omega \dot{m}}{4 \rho\left(z_{s}\right)} \sum_{m=1}^{\infty} u_{m}\left(z_{s}\right) u_{m}(z) H_{0}^{(1)}\left(k_{r n} r\right) .
$$

By utilizing the relationship between complex pressure and the velocity potential in Equation (1.49) an equivalent solution for the velocity potential may now be easily obtained. Applying this relationship to Equation (2.22), we arrive at the solution for the velocity potential due to a point source in a cylindrical waveguide with azimuthal symmetry:

$$
\hat{\Phi}(r, z)=\frac{i \dot{m}}{4 \rho\left(z_{s}\right) \rho(z)} \sum_{m=1}^{\infty} u_{m}\left(z_{s}\right) u_{m}(z) H_{0}^{(1)}\left(k_{r n} r\right) .
$$

Asymptotic forms of Equations (2.22) and (2.23) may be obtained by recalling the asymptotic form of $H_{0}^{(1)}\left(k_{r n} r\right)$ given in Equation (1.13). This leads to the following approximate solutions:

$$
\hat{p}(r, z) \approx-\frac{\omega \dot{m}}{\sqrt{8 \pi r} \rho\left(z_{s}\right)} e^{-i \pi / 4} \sum_{m=1}^{\infty} u_{m}\left(z_{s}\right) u_{m}(z) \frac{e^{i k_{r m} r}}{\sqrt{k_{r m}}}
$$

and

$$
\hat{\Phi}(r, z) \approx \frac{i \dot{m}}{\sqrt{8 \pi r} \rho\left(z_{s}\right) \rho(z)} e^{-i \pi / 4} \sum_{m=1}^{\infty} u_{m}\left(z_{s}\right) u_{m}(z) \frac{e^{i k_{r m} r}}{\sqrt{k_{r m}}} .
$$


Given Equations (2.24) and (2.25), we will now define a forward transmission loss equation:

$$
\operatorname{TL}(r, z)=-20 \log _{10}\left|\frac{\hat{p}(r, z)}{\hat{p}_{0}(r=1)}\right|=-20 \log _{10}\left|\frac{\hat{\Phi}(r, z)}{\hat{\Phi}_{0}(r=1)}\right|,
$$

where

$$
\hat{p}_{0}(r)=i \omega \dot{m} \frac{e^{i k_{0} r}}{4 \pi r}
$$

and

$$
\hat{\Phi}_{0}(r)=\frac{1}{\rho(z)} \dot{m} \frac{e^{i k_{0} r}}{4 \pi r},
$$

where $k_{0}=\omega / c_{0}$ and $c_{0}$ is a reference sound speed. Here $\hat{p}_{0}(r)$ and $\hat{\Phi}_{0}(r)$ represent reference values to be taken at $1 \mathrm{~m}$ from the source. Inserting either Equation (2.24) or (2.25) with its associated reference value into Equation (2.26), leads to the following approximate expression for forward transmission loss

$$
\mathrm{TL}(r, z) \approx-20 \log _{10}\left|\frac{1}{\rho\left(z_{s}\right)} \sqrt{\frac{2 \pi}{r}} \sum_{m=1}^{\infty} u_{m}\left(z_{s}\right) u_{m}(z) \frac{e^{i k_{r m} r}}{\sqrt{k_{r m}}}\right| .
$$

Equation (2.29) will be extended in later sections to compute forward transmission loss in a range-dependent shallow water environment.

\subsection{Wavenumber Decomposition and Evanescent Modes}

An intuitive discussion of the decomposition of wave phase in the normal modes solution can be had if we consider the case of an isovelocity flat-bottom waveguide with constant density. In this case, the modes have the general form:

$$
u_{m}(z)=A \sin \left(k_{z} z\right)+B \cos \left(k_{z} z\right)
$$

where

$$
k_{z}=\sqrt{\left(\frac{\omega}{c}\right)^{2}-k_{r}^{2}}
$$

is the vertical wavenumber and $k_{r}$ is the horizontal wavenumber. Recall the pressure-release surface boundary condition in Equation (2.7). This condition implies that $B=0$ in Equation (2.30). The rigid bottom boundary condition from 
Equation (2.7) suggests that either the coefficient $A$ must also be zero (i.e. the trivial solution) or that

$$
k_{z}=k_{z m}=\left(m-\frac{1}{2}\right) \frac{\pi}{H}, \quad m=1,2, \ldots
$$

Equation (2.32) further implies that $k_{r}$ must assume particular values, namely:

$$
k_{r}=k_{r m}=\sqrt{\left(\frac{\omega}{c}\right)^{2}-\left[\left(m-\frac{1}{2}\right) \frac{\pi}{H}\right]^{2}}, \quad m=1,2, \ldots,
$$

where $k_{r m}$ is recognized as the eigenvalue associated with mode $m$ and eigenfunction $u_{m}$. Combining Equations (2.31)-(2.33), we arrive at the expression:

$$
\left(\frac{\omega}{c}\right)=\sqrt{k_{r m}^{2}+k_{z m}^{2}}
$$

We recognize this quantity as the magnitude (norm) of the vector quantity $\boldsymbol{K}$, also known as the total wavenumber of the complex wave phase. If we let $\hat{x}$ and $\hat{z}$ be the unit base vectors along the $x$ and $z$ axes, respectively, we can write the total wavenumber in component form. For our two-dimensional problem of interest this is:

$$
\boldsymbol{K}=k_{r m} \hat{x}+k_{z m} \hat{z}
$$

Recall the asymptotic form of the Hankel function in Equation (1.13). It is easily verifiable from this equation that modes with positive imaginary eigenvalues are exponentially decaying in range. This allows us to partition the modes into propagating and evanescent (exponentially decaying/non-propagating) modes. For propagating modes (i.e. real $k_{r m}$ ) the following inequality holds:

$$
m<\frac{|\boldsymbol{K}| H}{\pi}+\frac{1}{2}
$$

Conversely, for evanescent modes (i.e. imaginary $k_{r m}$ ):

$$
m>\frac{|\boldsymbol{K}| H}{\pi}+\frac{1}{2}
$$


This decomposition of the total wavenumber has led to an interesting fact with regards to the modes in our isovelocity flat-bottom waveguide. Namely, the real eigenvalues and therefore the propagating modes have an upper bound of $|\boldsymbol{K}|=(\omega / c)$. As the source frequency is reduced the eigenvalues on the real axis slide to the left and up the imaginary axis. For a sufficiently low frequency, the first mode (i.e. $m=1$ ) will make this transition leaving no propagating modes in the problem. The frequency at which this occurs is known as the cutoff frequency for the waveguide and in appealing to the inequality in Equation (2.37), it is found to be:

$$
f_{c}=\frac{c}{4 H}
$$

Note that the depth of the waveguide also has an effect on which modes can propagate. As the waveguide becomes more shallow $H$ in the above inequalities becomes smaller in magnitude and lower order modes are cutoff. As was the case with frequency, there is a critical waveguide depth at which the first mode (i.e. $m=1$ ) will become cutoff leaving no propagating modes. Appealing again to Equation (2.37) we see that this depth is:

$$
H_{c}=\frac{c}{4 f}
$$

The decomposition of the total wavenumber allows for the discussion of another modal concept, that of mode grazing angle. We define the grazing angle of a mode as

$$
\theta_{m}=\arcsin \left(\frac{k_{z m}}{k}\right)=\arcsin \left(\frac{c k_{z m}}{\omega}\right),
$$

where $\theta_{m}$ corresponds to the angle that an equivalent ray associated with mode $m$ would make with the bottom boundary of the waveguide. For a flat-bottom waveguide $k_{z m}$ is constant for a particular mode $m$, as evident from Equation (2.31). If sound speed is also constant, the only quantity that can change in the 
argument to the arcsine function in Equation (2.40) is the angular frequency of the source, $\omega$. Decreasing the frequency of the source will result in an increase in the argument in Equation (2.40) thus pushing the arcsine toward its maximum real value of $90^{\circ}$.

As previously discussed, decreasing the source frequency also pushes the modes in a waveguide toward their cutoff. This means that the closer a mode is to its cutoff the steeper its grazing angle, $\theta_{m}$, will be. Once a mode is fully cutoff (i.e. its eigenvalue is purely imaginary), the real part of its grazing angle reaches $90^{\circ}$ and the mode is said to have "turned around." Steep-angle modes will be of particular interest in later sections of the presented work.

\subsection{Modal Velocities}

Another useful concept is modal velocity. To understand this concept consider a traveling wave of amplitude $A$ and a single frequency $f$. Such a wave may be represented by the sinusoid:

$$
A \cos (k x-\omega t)
$$

After some small unit of time $d t$ this wave will have experienced $\omega t / 2 \pi=f t$ oscillations. Over this same time window, an initial peak of the wave will have propagated a distance $d x$ away from the source to make room for this same number of oscillations (i.e. $k d x=\omega d t$ ). Recognizing this relationship allows us to define the following:

$$
c_{p}=\frac{d x}{d t}=\frac{\omega}{k}
$$

where $c_{p}$ is referred to as the phase velocity of the wave. The phase velocity represents the horizontal velocity of a phase point of the wave. It does not represent the speed of energy transport, which must be less than or equal to the speed of sound in the medium, $c$.

To obtain the speed of energy transport consider the combination of two waves 
with slightly different frequencies and wave lengths:

$$
\begin{aligned}
& \cos [(k-\Delta k) x-(\omega-\Delta \omega) t]+\cos [(k+\Delta k) x-(\omega+\Delta \omega) t] \\
& =2 \cos (\Delta k x-\Delta \omega t) \cos (k x-\omega t)
\end{aligned}
$$

The amplitude of the wave in Equation (2.43) is a sinusoidal envelope with phase speed $\Delta \omega / \Delta k$. In the limit, we refer to this speed as the group velocity

$$
c_{g}=\frac{d \omega}{d k}
$$

which is the speed at which energy is transported by a packet of waves.

The ratio between the speed of sound in the medium and the phase velocity of a wave propagating in the medium is known as the refractive index, $n=c / c_{p}=c k / \omega$. If one takes the derivative of $\omega=c k / n$ with respect to the wavenumber, $k$, we obtain

$$
\begin{aligned}
c_{g}=\frac{d \omega}{d k} & =\frac{c}{n}-\left(\frac{c k}{n^{2}} \times \frac{d n}{d k}\right) \\
& =c_{p}-\left(\frac{c k}{n^{2}} \times \frac{d n}{d k}\right) .
\end{aligned}
$$

As is evident from Equation (2.45), the only way for the group velocity and phase velocity to be equal is for the refractive index to be constant. In this case, the phase and group velocities are independent of frequency and the medium is said to be non-dispersive. Otherwise, the medium is said to be dispersive, meaning that a wave will separate into its individual frequency components while propagating through the medium due to the difference between the phase and group velocities.

Now we would like to connect the concept of phase and group velocity to the normal modes of propagation of an acoustic waveguide. At high frequencies all modes in an acoustic waveguide approach a propagating eigenvalue, which approaches the medium wavenumber [2]. That is,

$$
k_{r m} \rightarrow k \quad \text { as } \quad \omega \rightarrow \infty .
$$


Taking this into account and recalling Equation (2.42), we define the phase velocity of an individual mode $m$ as

$$
c_{p m}=\frac{\omega}{k_{r m}} .
$$

For steep-angle modes (i.e. those near cutoff), the phase velocity approaches infinity. Conversely, as the grazing angle of a mode becomes more horizontal (i.e. more propagating) the phase velocity approaches $c$, the speed of sound in the waveguide $[3]$.

Similar to the case for phase velocity, we can define a modal group velocity by taking into account Equations (2.46) and (2.44)

$$
c_{g m}=\frac{d \omega}{d k_{r m}} .
$$

The modal group velocity is the speed of energy transport of a particular mode. As the frequency of the acoustic source approaches the cutoff frequency for the mode its group velocity will approach zero. Conversely, as the frequency of the source approaches infinity, the group velocities of all modes in the waveguide will approach the speed of sound. Further investigation into the effects of horizontally-variable waveguide depth on modal group velocity has been documented in Appendix A.

\subsection{Solution via Cauchy's Residue Theorem}

For a point source in a medium with well-defined upper and lower boundaries that reflect the energy associated with an acoustic wave, we can define two solu-

tions, $u^{+}(z)$ and $u^{-}(z)$, of the homogeneous depth-separated equation in Equation (2.6). Here $u^{+}(z)$ accounts for the sum of the upper traveling wave plus the wave reflected from the upper boundary. Conversely, $u^{-}(z)$ accounts for the lower traveling wave plus the wave reflected from the lower boundary. Mathematically these solutions are given by the expressions

$$
u^{+}(z)=e^{i \int_{z}^{z_{1}} k_{z} \mathrm{~d} z}+R_{1} e^{-i \int_{z}^{z_{1}} k_{z} \mathrm{~d} z}
$$


and

$$
u^{-}(z)=e^{i \int_{z}^{z_{2}} k_{z} \mathrm{~d} z}+R_{2} e^{-i \int_{z}^{z_{2}} k_{z} \mathrm{~d} z}
$$

where $z_{2}>z_{s}>z_{1}$ and $z_{s}$ is the depth of the source. The vertical wavenumber, $k_{z}$, in these solutions is given by the expression in Equation (2.31), and $R_{1}$ and $R_{2}$ are reflection coefficients at the upper and lower boundaries of the medium, respectively.

The solutions in Equations (2.49) and (2.50) are known as Wentzel-KramersBrillouin, or WKB, solutions to the depth-separated wave equation. An alternative expression for the WKB solution is

$$
u(z)=\frac{1}{\sqrt{\eta \sin \theta}} e^{ \pm i k_{0} \int \eta \sin \theta \mathrm{d} z}
$$

where $c_{0}=\omega / k_{0}$ is a reference sound speed at the source location, $\eta=c_{0} / c$ is the diffraction coefficient due to depth-variable sound speed, and $\theta$ is the grazing angle of a ray associated with the solution $u(z)$ to the depth-separated equation. When written in this form we see that the solution blows up when $\theta=0$. This is because when $\theta$ is zero the equivalent ray associated with the solution $u(z)$ becomes horizontal and consequently turns over. As such, we say that the WKB approximation breaks down at turning points. Further details on the WKB solution and the discipline of geometric acoustics can be found in reference [2].

Recalling the method of variation of parameters, we can derive a particular solution to the inhomogeneous equation corresponding to Equation (2.6). This solution is of the form of an integral product of a piece-wise defined Green's function for our medium of interest and the forcing term on the right side of the depthseparated equation. The Green's function has the form:

for $z \leq z_{s}$ :

$$
G\left(z, z_{s}, k_{r}^{2}\right)=\frac{u^{+}(z) u^{-}\left(z_{s}\right)}{W\left(u^{+}, u^{-}, k_{r}^{2}\right)},
$$


for $z \geq z_{s}$ :

$$
G\left(z, z_{s}, k_{r}^{2}\right)=\frac{u^{-}(z) u^{+}\left(z_{s}\right)}{W\left(u^{+}, u^{-}, k_{r}^{2}\right)},
$$

where $W\left(u^{+}, u^{-}, k_{r}^{2}\right)$ is the Wronskian of the two chosen solutions to the homogeneous equation, defined as:

$$
W\left(u^{+}, u^{-}, k_{r}^{2}\right)=u^{+}\left(z_{s}\right) u^{\prime-}\left(z_{s}\right)-u^{-}\left(z_{s}\right) u^{\prime+}\left(z_{s}\right),
$$

where primes denote differentiation with respect to $z$. Note, the Wronskian is constant for a given value of $k_{r}$. Next we observe that a normal mode will exist when the upward traveling and downward traveling waves superimpose to create a standing wave within the waveguide. This condition implies linear dependence of the two solutions $u^{+}(z)$ and $u^{-}(z)$, which will occur when $W\left(u^{+}, u^{-}, k_{r}^{2}\right)=0$.

In general, the sum of our particular solution to the inhomogeneous equation with the general solution of the homogeneous equation (i.e. $u_{h}=u^{+}(z)+u^{-}(z)$ ) will give us the total solution to the depth-separated equation. If we then combine this solution with the solution to the range-separated equation we can express the total solution to the wave equation in Equation (2.1) as a contour integral in wavenumber space. Thus we have:

$$
\hat{p}(r, z)=\oint H_{0}^{(1)}\left(k_{r} r\right) G\left(z, z_{s}, k_{r}^{2}\right) k_{r} \mathrm{~d} k_{r}
$$

The previous statement regarding the conditions for the existence of normal modes implies that values of $k_{r}$ corresponding to normal modes represent singularities (poles) in the integrand of Equation (2.55). To evaluate this integral we apply Cauchy's Residue Theorem [4] to express its solution as a sum of the individual residues corresponding to each normal mode. Further details on this normal modes approach can be found in reference [3]. 


\subsection{Range-Dependent Normal Modes}

The introduction of range-dependence into the conditions of Equation (2.2) significantly increases the difficulty of finding a solution for the acoustic field. This is primarily due to the fact that the modes of vibration of the wave equation become a function of depth and range. This means that our assumed solution in Equation (2.10) takes the form

$$
\hat{p}(r, z)=\sum_{m=1}^{\infty} \varphi_{m}(r) u_{m}(r, z) .
$$

Inserting this proposed solution into Equation (2.2) and following a procedure

similar to that for the canonical normal modes derivation leads to the following set of coupled equations for the expansion coefficients $\varphi_{m}(r)$ :

$$
\frac{1}{r} \frac{d}{d r}\left(r \frac{d \varphi_{n}}{d r}\right)+\sum_{m=1}^{\infty} 2 B_{m n} \frac{d \varphi_{m}}{d r}+\sum_{m=1}^{\infty} A_{m n} \varphi_{m}+k_{r n}^{2}(r) \varphi_{n}=-i \omega \dot{m} \frac{\delta(r) u_{n}\left(r, z_{s}\right)}{\rho\left(z_{s}\right) 2 \pi r}
$$

where

$$
A_{m n}=\int \frac{1}{r} \frac{\partial}{\partial r}\left(r \frac{\partial u_{m}}{\partial r}\right) \frac{u_{n}}{\rho} \mathrm{d} z
$$

and

$$
B_{m n}=\int \frac{\partial u_{m}}{\partial r} \frac{u_{n}}{\rho} \mathrm{d} z
$$

The expressions for the coefficients in Equations (2.58) and (2.59) involve modes of mixed index. This is due to the fact that in range-dependent environments energy is transferred between adjacent modes. This non-adiabatic energy transference, known as mode coupling, prevents the use of a classical normal modes approach to solving for the acoustic field.

\subsection{Previous Work}

A number of approaches have been developed for treating the range-dependent problem described in Section (2.6). One of the first solutions to this problem was described by Pierce [5] in 1964. Known as the adiabatic assumption in mode cou- 
pling theory, this approach assumes a nearly stratified acoustic medium such that coupling between modes may be neglected. The justification for this simplification is that, for sufficiently slowly varying media, the terms involving the coupling coefficients $A_{m n}$ and $B_{m n}$ in Equation (2.57) are small and may be neglected. Milder [6] presented a similar argument in 1969 based on the proposition that the equivalent rays associated with the normal modes of acoustic propagation are invariant in acoustic media with mild range-dependence. While this adiabatic approach to range-dependent acoustic propagation has been justified in a number of cases, there are still many other propagation scenarios for which the neglect of non-adiabatic mode coupling leads to significant errors in the estimation of the acoustic field. For example, Godin [7] showed that ignoring coupling between modes leads to a violation of conservation of energy and reciprocity principles in the presence of a sloping interface or a range-dependent rigid boundary.

Most approaches to the range-dependent problem that account for mode coupling fall into one of three classes. In the first class of solutions one attempts to directly solve the coupled equation given in Equation (2.57). Among this family of solutions is the approach described by Buckingham [8] in 1987. In this approach, the author transforms the acoustic wave equation into a cylindrical coordinate system where the $z$ axis is horizontal, termed the "wedge coordinate system." For the case of an isovelocity waveguide, it is then possible to derive a set of uncoupled "wedge modes," which may be summed to approximate the acoustic field. In 1991 Primack and Gilbert [9] extended the work of Buckingham by developing a computational model based on this wedge modes approach. They showed that for realistic sound-speed profiles coupling between wedge modes must be included in the calculation of the field. Fawcett, Westwood, and Tindle [10] further extended the work of Primack and Gilbert by including leaky modes into the field calculation and de- 
veloping a least-squares approximation for computing mode coupling in the wedge coordinate system. While this wedge mode approach to range-dependent acoustic propagation shows promise for single-slope propagation scenarios, its applicability to general range-dependent media requires further development.

Also belonging to this first class of solutions to the range-dependent problem is the work of Desaubies, Chiu, and Miller [11]. In their 1986 study these authors set out to quantify when it is appropriate to make the adiabatic approximation in the context of slow changes to the sound-speed structure of the ocean due to mesoscale eddies. In doing so, they present a simplification to the coupled system given by Equations (2.57)-(2.59) based on an adiabatic expansion of the range-dependent wavenumber. It was ultimately determined in this study that the adiabatic solution fails for environments with range-dependent sound speed under the conditions of high frequency, high mode number, and long range. Note, the approach of this study assumes a flat-bottom waveguide and therefore is limited to environments where sound speed is the dominant mechanism for range dependence. Chiu, Miller, and Lynch [12] also set out to solve the range-dependent problem via directly solving Equation (2.57) in 1996. In their approach a fourth-order finite difference method is utilized for approximating the first and second depth derivatives of the eigenfunctions associated with the modes of acoustic propagation. Fourth and fifth-order Runge-Kutta algorithms are used to solve a coupled system of equations governing complex envelopes of the mode amplitudes. The pressure field is ultimately resolved via an inverse Fourier transform of the product of the source signal spectrum and a source-to-receiver ocean transfer function. Computational savings are achieved in this approach through computing the gradually varying complex envelopes of the modal amplitudes rather than the rapidly varying mode amplitudes themselves. Conversely, computational bottlenecks are found in the 
method's need to numerically solve the integrals associated with the coupling coefficients and in the iterative approach (i.e. Runge-Kutta) required to solve the coupled differential equation governing the modal envelopes. Other direct solutions to the range-dependent wave equation can be found in works by Boyles [13], Gillette [14], and Stotts [15].

The second class of solutions to the problem described in Section (2.6) includes approximate methods whereby assumptions are made that lead to simplifications in the range-dependent acoustic wave equation. These methods are often more computationally efficient due to their simplicity; however, this gain in efficiency is often at the cost of accuracy in environments with severe range-dependence. The family of methods known as parabolic-equation methods belong to this class of approximate solutions. The parabolic-equation method was first introduced into underwater acoustics in the early 1970s by Hardin and Tappert [16]. In these solutions, one begins with a Helmholtz equation of the form

$$
\frac{\partial^{2} p}{\partial r^{2}}+\frac{1}{r} \frac{\partial p}{\partial r}+\frac{\partial^{2} p}{\partial z^{2}}+k_{0}^{2} n^{2} p=0
$$

where $n(r, z)=c_{0} / c(r, z)$ is the index of refraction and $c_{0}$ is a reference sound speed. A solution of the form $p(r, z)=\psi(r, z) H_{0}^{(1)}\left(k_{0} r\right)$ is assumed and inserted into Equation (2.60). Next, it is assumed that $k_{0} r \gg 1$ (i.e. the farfield assumption), resulting in the differential equation:

$$
\frac{\partial^{2} \psi}{\partial r^{2}}+2 i k_{0} \frac{\partial \psi}{\partial r}+\frac{\partial^{2} \psi}{\partial z^{2}}+k_{0}^{2}\left(n^{2}-1\right) \psi=0
$$

From here, the following paraxial approximation (also known as the small angle approximation) is made:

$$
\frac{\partial^{2} \psi}{\partial r^{2}} \ll 2 i k_{0} \frac{\partial \psi}{\partial r}
$$

This ultimately leads to what is known as the parabolic equation of underwater 
acoustics:

$$
2 i k_{0} \frac{\partial \psi}{\partial r}+\frac{\partial^{2} \psi}{\partial z^{2}}+k_{0}^{2}\left(n^{2}-1\right) \psi=0 .
$$

Methods in this family of solutions are then focused upon solving Equation (2.63). While the parabolic equation given in Equation (2.63) is significantly easier to solve than the quadratic acoustic wave equation, this approach comes with some caveats. In addition to the farfield assumption, the paraxial approximation in Equation (2.62) puts additional constraints on the propagation scenarios that may be considered. In particular, parabolic-equation methods require that back scattering be negligible and that range-dependence in the acoustic medium be relatively weak. These methods also constrain the propagation scenario to angles less than or equal to about ten to fifteen degrees off the horizontal and to high source frequencies.

Tindle, O'Driscoll, and Higham [17] developed a method in 2000 that also belongs to the class of approximate solutions to the range-dependent wave equation. In their approach the effects of mode coupling due to range-dependent sound speed are accounted for through a novel application of perturbation theory. Mode coupling is represented via small perturbative terms that are added to the eigenfunctions and eigenvalues associated with the modes. These perturbed eigenfunctions and eigenvalues are then used in the canonical (i.e. range-independent) normal modes solution to approximate the acoustic field. Tindle and Higham later extended this approach to account for coupling due to range-dependent density [18] and internal waves [19]. Kampanis and Dougalis [20] developed an approximate method for solving the range-dependent wave equation based on the Galerkin finite element method in 1999. In 2005 Clark [21] extended a multipath expansion method for solving the Helmholtz wave equation, originally developed by Leibiger [22], to account for horizontal variations in bottom depth, bottom type, and sound 
speed via a stationary phase approximation. A deep water variation of this method that maintains continuity through mode turning points was published by Clark and Smith in 2008 [23].

In the third class of solutions to the problem of range-dependent acoustic propagation the horizontally-variable acoustic environment is approximated via a series of range-independent steps. Within each of these steps a canonical normal modes solution such as the one described in Sections (2.2) and (2.5) may be applied. The non-adiabatic transfer of energy between modes is then accounted for by applying continuity conditions at discrete interfaces separating the steps. This approach first appears in a dissertation by Leibiger [22] in 1968. In this work, Leibiger develops an asymptotic theory for estimating transmission and reflection coefficients at discrete interfaces throughout the range-dependent medium by writing the eigenfunctions and eigenvalues as an expansion in range. This theory is developed in the context of near-surface propagation in an infinitely deep ocean and therefore coupling effects due to a sloping seafloor are neglected. Moreover, the transmission and reflection coefficient solutions developed in this work are limited by the assumption that the wavefronts of the acoustic excitation are planar (i.e. the farfield assumption). Evans [24] developed a more general stepwise coupled mode approach in 1983. This solution was implemented into a computational model known as COUPLE and is considered a benchmark solution for problems in range-dependent acoustic propagation. Despite its ability to provide an exact solution, the computational intensity required by the COUPLE solution makes it unsuitable for real-time or near real-time applications. Instead, it often serves as a benchmark for less computationally intensive approximate solutions [3]. 


\section{List of References}

[1] G. N. Watson, A Treatise on the Theory of Bessel Functions, 2nd ed. New York: Cambridge University Press, 1995.

[2] C. A. Boyles, Acoustic Waveguides: Applications to Oceanic Science. New York: Wiley-Interscience, 1984.

[3] F. B. Jensen, W. A. Kuperman, M. B. Porter, and H. Schmidt, Computational Ocean Acoustics, 1st ed. New York: Springer-Verlag, 2000.

[4] J. W. Brown and R. V. Churchill, Complex Variables and Applications, 7th ed. New York: McGraw-Hill, 2003.

[5] A. D. Pierce, "Extension of the method of normal modes to sound propagation in an almost-stratified medium," Journal of the Acoustical Society of America, vol. 37, no. 1, pp. 19-27, 1964.

[6] D. M. Milder, "Ray and wave invariants for sofar channel propagation," Journal of the Acoustical Society of America, vol. 46, no. 5, pp. 1259-1263, 1969.

[7] O. A. Godin, "A note on differential equations of coupled-mode propagation in fluids," Journal of the Acoustical Society of America, vol. 103, no. 1, pp. 159-168, 1998.

[8] M. J. Buckingham, "Theory of three-dimensional acoustic propagation in a wedgelike ocean with a penetrable bottom," Journal of the Acoustical Society of America, vol. 82, no. 1, pp. 198-210, 1987.

[9] H. Primack and K. E. Gilbert, "A two-dimensional downslope propagation model based on coupled wedge modes," Journal of the Acoustical Society of America, vol. 90, no. 6, pp. 3254-3262, 1991.

[10] J. A. Fawcett, E. K. Westwood, and C. T. Tindle, "A simple coupled wedge mode propagation method," Journal of the Acoustical Society of America, vol. 98, no. 3, pp. 1673-1681, 1995.

[11] Y. Desaubies, C.-S. Chiu, and J. H. Miller, "Acoustic mode propagation in a range-dependent ocean," Journal of the Acoustical Society of America, vol. 80, no. 4, pp. 1148-1160, 1986.

[12] C.-S. Chiu, J. H. Miller, and J. F. Lynch, "Forward coupled-mode propagation modeling for coastal acoustic tomography," Journal of the Acoustical Society of America, vol. 99, no. 2, pp. 793-802, 1996.

[13] C. A. Boyles, "Coupled mode solution for a cylindrically symmetric oceanic waveguide with a range and depth dependent refractive index and a time varying rough sea surface," Journal of the Acoustical Society of America, vol. 73, no. 3, pp. 800-805, 1983. 
[14] G. Gillette, "Coupled modes in a waveguide with a range-dependent rigid basement," Journal of the Acoustical Society of America, vol. 95, no. 1, pp. 187-200, 1994.

[15] S. A. Stotts, "Coupled-mode solutions in generalized ocean environments," Journal of the Acoustical Society of America, vol. 111, no. 4, pp. 1623-1643, 2002.

[16] R. H. Hardin and F. D. Tappert, "Applications of the split-step fourier method to the numerical solution of nonlinear and variable coefficient wave equations," SIAM Rev., vol. 15, p. 423, 1973.

[17] C. T. Tindle, L. M. O'Driscoll, and C. J. Higham, "Coupled mode perturbation theory of range dependence," Journal of the Acoustical Society of America, vol. 108, no. 1, pp. 76-83, 2000.

[18] C. J. Higham and C. T. Tindle, "Coupled perturbed modes over a sloping penetrable bottom," Journal of the Acoustical Society of America, vol. 114, no. 6, pp. 3119-3124, 2003.

[19] C. J. Higham and C. T. Tindle, "Coupled perturbed modes and internal solitary waves," Journal of the Acoustical Society of America, vol. 113, no. 5, pp. 2515-2522, 2003.

[20] N. A. Kampanis and V. A. Dougalis, "A finite element code for the numerical solution of the Helmholtz equation in axially symmetric waveguides with interfaces," Journal of Computational Acoustics, vol. 7, no. 2, pp. 83-110, 1999.

[21] C. A. Clark, "Acoustic wave propagation in horizontally variable media," IEEE Journal of Oceanic Engineering, vol. 30, no. 1, pp. 188-197, 2005.

[22] G. A. Leibiger, "Wave propagation in an inhomogeneous medium with slow spatial variation," Ph.D. dissertation, Stevens Institute of Technology, Castle Point, Hoboken, New Jersey, 1968.

[23] C. A. Clark and K. B. Smith, "An efficient normal mode solution to wave propagation prediction," IEEE Journal of Oceanic Engineering, vol. 33, no. 4, pp. 462-476, 2008.

[24] R. B. Evans, "A coupled mode solution for acoustic propagation in a waveguide with stepwise depth variations of a penetrable bottom," Journal of the Acoustical Society of America, vol. 74, no. 1, pp. 188-195, 1983. 


\section{CHAPTER 3}

\section{Asymptotic Stepwise Coupled Modes}

\subsection{Introduction}

The remaining goal of this dissertation is to develop a non-adiabatic mode coupling solution suitable for implementation in a computational model for the prediction of acoustic energy propagation in range-dependent shallow water environments. Particular attention will be given to developing a solution that maintains analytical integrity while offering an increase in computational efficiency over currently existing solutions. This will be accomplished through a novel two-layer discretization of the environment, which leads to closed-form expressions for computing non-adiabatic mode coupling at discrete interfaces throughout the acoustic medium. This approach depends upon writing the range-dependent eigenfunctions and eigenvalues as an expansion in range. Doing so leads to the development of a range-expanded normal mode inner product for estimating the transfer of energy between modes upon encountering a horizontal change in environment. Further efficiency in the presented solution is gained through the extension and application of an asymptotic coupled mode theory originally developed for near-surface propagation in an infinitely deep ocean, which is documented in reference [1].

We begin by assuming an environment in which the horizontal changes in the acoustic properties of the medium are small over a distance equal to the wavelength of the source frequency. A medium possessing this quality will be termed slowly varying. This assumption will be coupled with the assumption that the acoustic velocity potential may be expanded locally as a sum of normal modes, i.e.,:

$$
\hat{\Phi}(x, y, z)=\sum_{n=1}^{\infty} \varphi_{n}(x, y) u_{n}(z ; x, y)
$$


where the $u_{n}(z ; x, y)$ are the solutions of the Sturm-Liouville problem

$$
\frac{d^{2} u}{d z^{2}}+\left(\frac{\omega^{2}}{c^{2}(z ; x, y)}-k_{r}^{2}\right) u=0
$$

with boundary conditions

$$
\begin{aligned}
u(0) & =0 \\
\left.\frac{d u(z)}{d z}\right|_{z=H} & =0 .
\end{aligned}
$$

Here, the range coordinates $(x, y)$ are fixed and the depth coordinate, $z$, is measured positive-down.

The form of the proposed solution in Equation (3.1) suggests that the propagation in a slowly varying medium is by modes, although the horizontally variable character of the medium serves to perturb the eigenvalues, $k_{n}$, and the eigenfunctions, $u_{n}(z ; x, y)$, defining these modes. Intuitive justification for this approach is found in the fact that even though the individual modes experience this perturbation, the slowly variable nature of the medium should not have strong effects on the energy transport through the medium. This implies that such transport is essentially the same in character as that of the canonical normal modes problem.

\subsection{The Range-Expanded Normal Mode Inner Product}

For simplification of the following discussion we will turn our attention to the two-dimensional problem. In doing so, the acoustic field will be considered a function of the spatial coordinates $x$ and $z$, where these coordinates represent range and depth, respectively. The property of the slowly varying range-dependent medium to perturb the eigenvalues and eigenfunctions is then introduced into the analysis by assuming the validity, at least to second order, of the following representation:

$$
\begin{gathered}
k_{n}(x+\Delta x)=k_{n}(x)+k_{n}^{\prime}(x) \Delta x+\frac{1}{2} k_{n}^{\prime \prime}(x)(\Delta x)^{2}+\ldots \\
u_{n}(z, x+\Delta x)=u_{n}(z, x)+u_{n}^{\prime}(z, x) \Delta x+\frac{1}{2} u_{n}^{\prime \prime}(z, x)(\Delta x)^{2}+\ldots,
\end{gathered}
$$


where primes denote differentiation with respect to $x$. Thus Equations (3.4) and (3.5) provide expressions for the perturbed eigenvalues and eigenfunctions, respectively, upon taking a single range step of size $\Delta x$. It is next noted that due to the orthonormality of the original eigenfunctions, $u_{n}(z, x)$, the perturbed eigenfunctions, $u_{n}(z, x+\Delta x)$, may be expressed as:

$$
\begin{gathered}
u_{n}(z, x+\Delta x)=\sum_{m=1}^{N}\left\langle u_{n}(z, x+\Delta x), u_{m}(z, x)\right\rangle u_{m}(z, x), \\
n=1,2, \ldots, N
\end{gathered}
$$

where $\langle f(z, x), g(z, x)\rangle$ denotes the inner product

$$
\langle f(z, x), g(z, x)\rangle=\int_{0}^{H(x)} f(z, x) g(z, x) \mathrm{d} z,
$$

and $H(x)$ is the bottom depth from the surface at range $x$, which may be either finite or infinite.

In proceeding from range $x$ to range $x+\Delta x$, this transformation imposes a rotation of the basis vectors of the appropriate function space, or domain of the differential operator from Equation (3.2). The inner product in Equation (3.6) then describes the amount of energy transferred from each of the modes $m$ to mode $n$ upon taking this step in range. Geometrically, this inner product can be interpreted as the projection of mode $n$ at range $x+\Delta x$ onto each of the modes $m$ at range $x$. Substituting Equation (3.5) into the inner product in Equation (3.6), we arrive at the following range-expanded normal mode inner product describing the coupling between modes $m$ and $n$ :

$$
\begin{aligned}
\left\langle u_{n}(z, x+\Delta x),\right. & \left.u_{m}(z, x)\right\rangle \\
& \approx\left\langle u_{n}(z, x), u_{m}(z, x)\right\rangle+\left\langle u_{n}^{\prime}(z, x), u_{m}(z, x)\right\rangle \Delta x \\
& +\frac{1}{2}\left\langle u_{n}^{\prime \prime}(z, x), u_{m}(z, x)\right\rangle(\Delta x)^{2}+\ldots
\end{aligned}
$$

There are three important properties of the above range-expanded normal mode inner product that will be utilized in further derivation of the presented 
theory. The first two of these properties relate the value of the inner product for coupling due to forward propagating acoustic energy to that for backwards propagation. We will derive these properties by first recalling the definition of the Taylor series for a real or complex-valued function $f(t)$ that is infinitely differentiable at a real or complex number, $a$ :

$$
f(t) \approx f(a)+\frac{f^{\prime}(a)}{1 !}(t-a)+\frac{f^{\prime \prime}(a)}{2 !}(t-a)^{2}+\frac{f^{\prime \prime \prime}(a)}{3 !}(t-a)^{3}+\ldots
$$

For the case of forward coupling, let $t=(x+\Delta x)$ and $a=x$ in Equation (3.9) and let the function $f$ be the eigenfunctions, $u_{n}$, associated with the modes of vibration of the acoustic medium. In doing so, we see that $u_{n}(z, x+\Delta x)$ is equal to the expression given in Equation (3.5), which when inserted into the inner product in Equation (3.6) leads to the expression in Equation (3.8) for the forward range-expanded normal mode inner product. Following a similar procedure for the case of backwards coupling, we let $t=(x-\Delta x)$ and $a=x$ in Equation (3.9) and again let $f$ correspond to the eigenfunctions, $u_{n}$. In doing so, we arrive at an expression for the backwards range-expanded normal mode inner product:

$$
\begin{aligned}
\left\langle u_{n}(z, x-\Delta x),\right. & \left.u_{m}(z, x)\right\rangle \\
& \approx\left\langle u_{n}(z, x), u_{m}(z, x)\right\rangle-\left\langle u_{n}^{\prime}(z, x), u_{m}(z, x)\right\rangle \Delta x \\
& +\frac{1}{2}\left\langle u_{n}^{\prime \prime}(z, x), u_{m}(z, x)\right\rangle(-\Delta x)^{2}+\ldots
\end{aligned}
$$

If we now restrict ourselves to the case of $m \neq n$, we see that the first term in both Equations (3.8) and (3.10) cancel due to the orthogonality of the eigenfunctions. We will further specify that $\Delta x$ is small and therefore second and higher order terms in these expansions are negligible. Under these assumptions we see that

$$
\begin{aligned}
\left\langle u_{n}(z, x+\Delta x), u_{m}(z, x)\right\rangle & \approx\left\langle u_{n}^{\prime}(z, x), u_{m}(z, x)\right\rangle \Delta x \\
m & \neq n
\end{aligned}
$$


and

$$
\begin{aligned}
\left\langle u_{n}(z, x-\Delta x), u_{m}(z, x)\right\rangle & \approx-\left\langle u_{n}^{\prime}(z, x), u_{m}(z, x)\right\rangle \Delta x . \\
m & \neq n
\end{aligned}
$$

Taking into account Equations (3.11) and (3.12), we finally arrive at the first desired property:

$$
\begin{aligned}
\left\langle u_{n}(z, x+\Delta x), u_{m}(z, x)\right\rangle & \approx-\left\langle u_{n}(z, x-\Delta x), u_{m}(z, x)\right\rangle . \\
m & \neq n
\end{aligned}
$$

This property states that for modes of mixed index (i.e. cross coupling) the forward and backward inner product differ by a sign. A similar procedure can be applied to the case $m=n$, leading to the second desired property between the forward and backward inner products:

$$
\left\langle u_{n}(z, x+\Delta x), u_{n}(z, x)\right\rangle \approx-\left\langle u_{n}(z, x-\Delta x), u_{n}(z, x)\right\rangle+2
$$

In deriving the third property of interest we must first recall the Leibniz Integral Rule, which provides the value of the derivative of an integral whose limits of integration are a function of the variable of differentiation. The formal statement of this rule says that if a function $f(z, x)$ exists such that its partial derivative with respect to the variable $x$ exists and is continuous then,

$\frac{\partial}{\partial x}\left(\int_{a(x)}^{b(x)} f(z, x) \mathrm{d} z\right)=f(b(x), x) \bullet b^{\prime}(x)-f(a(x), x) \bullet a^{\prime}(x)+\int_{a(x)}^{b(x)} \frac{\partial}{\partial x} f(z, x) \mathrm{d} z$.

In applying this rule to acoustic propagation via modes in a range-dependent waveguide we note that $a(x)=0$ and $b(x)=H(x)$, where we recall that $H(x)$ is the range-dependent bottom depth function. Next, we let the function in Equation (3.15) be $u_{n}^{2}(z, x)$, such that the integral on the left side of this equation is the inner product $\left\langle u_{n}, u_{n}\right\rangle$. In doing so, we recognize that this integral evaluates to unity due to orthogonality of the eigenfunctions and therefore the entire term vanishes. 
Applying the product rule for differentiation to the integrand on the right side of Equation (3.15) and bringing the result over to the left side leads to the desired property:

$$
\left\langle u_{n}^{\prime}(z, x), u_{n}(z, x)\right\rangle=-\frac{1}{2} u_{n}^{2}(H(x), x) \cdot H(x) .
$$

Note that in reference [1] the left side of Equation (3.16) is stated to be equal to zero. This is due to the fact that variation in bottom depth with range can be neglected when considering near-surface propagation in a deep ocean waveguide. The effect of this assumption is most easily illustrated if we consider the simplified case of an isovelocity acoustic wedge with pressure-release surface and rigid bottom. For this case, the range-dependent eigenfunctions can be written as

$$
u_{n}(z, x)=\sqrt{\frac{2 \rho}{H(x)}} \sin \left[\left(n-\frac{1}{2}\right) \pi \frac{z}{H(x)}\right],
$$

where density, $\rho$, is constant. The corresponding range-dependent eigenvalues in this environment are given by the expression

$$
k_{n}(x)=\sqrt{\left(\frac{\omega}{c}\right)^{2}-\left(n-\frac{1}{2}\right)^{2}\left(\frac{\pi}{H(x)}\right)^{2}} .
$$

A closed-form expression can also be written for the range-dependent bottom depth function in the wedge waveguide. Namely,

$$
H(x)=H_{0}+x \tan \beta
$$

where $H_{0}$ is the initial bottom depth at the source and $\beta$ is the bottom slope angle. Inserting Equation (3.17) and (3.19) into right side of Equation (3.16) leads to

$$
\left\langle u_{n}^{\prime}(z, x), u_{n}(z, x)\right\rangle=-\frac{1}{H(x)} \tan \beta
$$

From this form of the property we can see that for a flat bottom (i.e. $\beta=0$ ) the right side of the equation vanishes. The implication of this property for small values of $\Delta x$ is that the inner product in Equation (3.8) is approximately unity 
for the case $m=n$ (i.e. direct coupling). This is due to the fact that all terms on the right side of Equation (3.8) except for the first are approximately zero. If, however, the bottom depth is range-dependent (i.e. $\beta \neq 0$ ) the term in Equation (3.16) must be included when evaluating the inner product for direct coupling.

It is important to note that the range-expanded normal mode inner product in Equation (3.8) describes the coupling between modes due to horizontal changes in the environment encountered when taking a single step in range. This inner product cannot be used to compute modes at successive range steps via Equation (3.6) as the condition of orthogonality is lost after successive steps in range due to estimation error accumulating in the approximated modes. Moreover, use of the inner product in Equation (3.8) to estimate mode coupling at range $x+\Delta x$ requires prior knowledge of the amplitudes and shapes of the orthonormal eigenfunctions corresponding to the modes at range $x$. Taking this into account, a method is desired for resolving the mode amplitudes and shapes, which implements the inner product in Equation (3.8) to account for non-adiabatic mode coupling at discrete steps through the medium. Furthermore, it is desirable that this method maintains analytical rigor and computational efficiency so as to remain compatible with realtime or near real-time applications. In the next section a discretization technique for the range-dependent medium is developed that provides a way forward.

\subsection{Discretization of the Range-Dependent Medium}

As previously stated, implementation of the inner product in Equation (3.8) for estimating non-adiabatic mode coupling requires prior knowledge of the amplitudes and shapes of the normal modes associated with the range-dependent acoustic medium. Once the modes have been resolved, we then need a means for implementing the inner product to account for the energy transport down range as a set of acoustic waves propagates through the medium. To address the resolu- 
tion of the modes a novel discretization approach is proposed whereby the general range-dependent environment is approximated by a series of connected simpler range-dependent subenvironments. These subenvironments are chosen such that their horizontal variability is simple enough to be captured within closed-form expressions for the eigenfunctions and eigenvalues corresponding to the modes that exist within each environment.

As an example, consider a hypothetical environment with constant sound speed and general range-dependent bottom depth, as depicted in Figure (3.1a). For simplicity of discussion we will further specify that this environment have a pressure-release surface and rigid bottom. Applying the proposed discretization technique, this environment may be approximated by a series of acoustic wedges as depicted in Figure(3.1). As discussed in Section (3.2), the range-dependent eigenfunctions for the isovelocity acoustic wedge with pressure-release surface and rigid bottom are given by the expression in Equation (3.17) and the range-dependent eigenvalues are given by Equation (3.18). Use of these expressions allows for the range-expanded normal mode inner product in Equation (3.8) to be computed in closed form for each of the acoustic wedges used to approximate the general environment. The terms of the range-expanded normal mode inner product for the acoustic wedge are given by the following closed-form expressions:

$$
\begin{gathered}
\text { For } m=n: \\
\left\langle u_{n}(z, x), u_{n}(z, x)\right\rangle=1 \\
\left\langle u_{n}^{\prime}(z, x), u_{n}(z, x)\right\rangle=-\frac{1}{H} \tan \beta \\
\left\langle u_{n}^{\prime \prime}(z, x), u_{n}(z, x)\right\rangle=\left(\frac{7}{4}-\frac{1}{3}\left(n-\frac{1}{2}\right)^{2} \pi^{2}\right) H^{-2} \tan ^{2} \beta \\
\text { For } m \neq n:
\end{gathered}
$$




$$
\begin{aligned}
\left\langle u_{n}(z, x), u_{m}(z, x)\right\rangle & =0 \\
\left\langle u_{n}^{\prime}(z, x), u_{m}(z, x)\right\rangle & =(-1)^{(m+n)} \frac{(2 n-1)^{2}}{2 H(m-n)(m+n-1)} \tan \beta \\
\left\langle u_{n}^{\prime \prime}(z, x), u_{m}(z, x)\right\rangle & =(-1)^{(m+n-1) \frac{(2 n-1)^{2}}{2 H^{2}}} \\
& \times\left[\frac{3}{(m-n)(m+n-1)}+\frac{(m+n-1)^{2}+(m-n)^{2}}{(m-n)^{2}(m+n-1)^{2}}\right] \\
& \times \tan ^{2} \beta .
\end{aligned}
$$

Additionally, each of the subenvironments used in this discretization are fully specified by the parameter $\beta$, the bottom slope angle, once the initial bottom depth at the acoustic source is known. This is evident through observance of the expression for the bottom depth function, $H(x)$, in Equation (3.19). Application of this discretization approach to more general environments necessitates thought as one must choose the subenvironments such that all mechanisms for range-dependence are captured in closed-form expressions for the eigenfunctions and eigenvalues corresponding to the modes of each environment. Once this is accomplished, however, significant computational gains are achieved as mode coupling is calculated via closed-form algebraic expressions. This differs from direct approaches to solving the range-dependent wave equation, which require numerically solving the integrals in Equations (2.58) and (2.59) to obtain coupling coefficients. An additional benefit of this approach is the elimination of the need to resolve the eigenvalues numerically, a computationally expensive problem that exists in many other solutions.

We now desire a method for incorporating the closed-form solutions for the range-expanded normal mode inner product obtained from this discretization approach into a calculation of energy transport through the range-dependent environment due to an acoustic excitation. To accomplish this we let any one of the subenvironments previously discussed (e.g. the wedge subenvironments in Fig- 


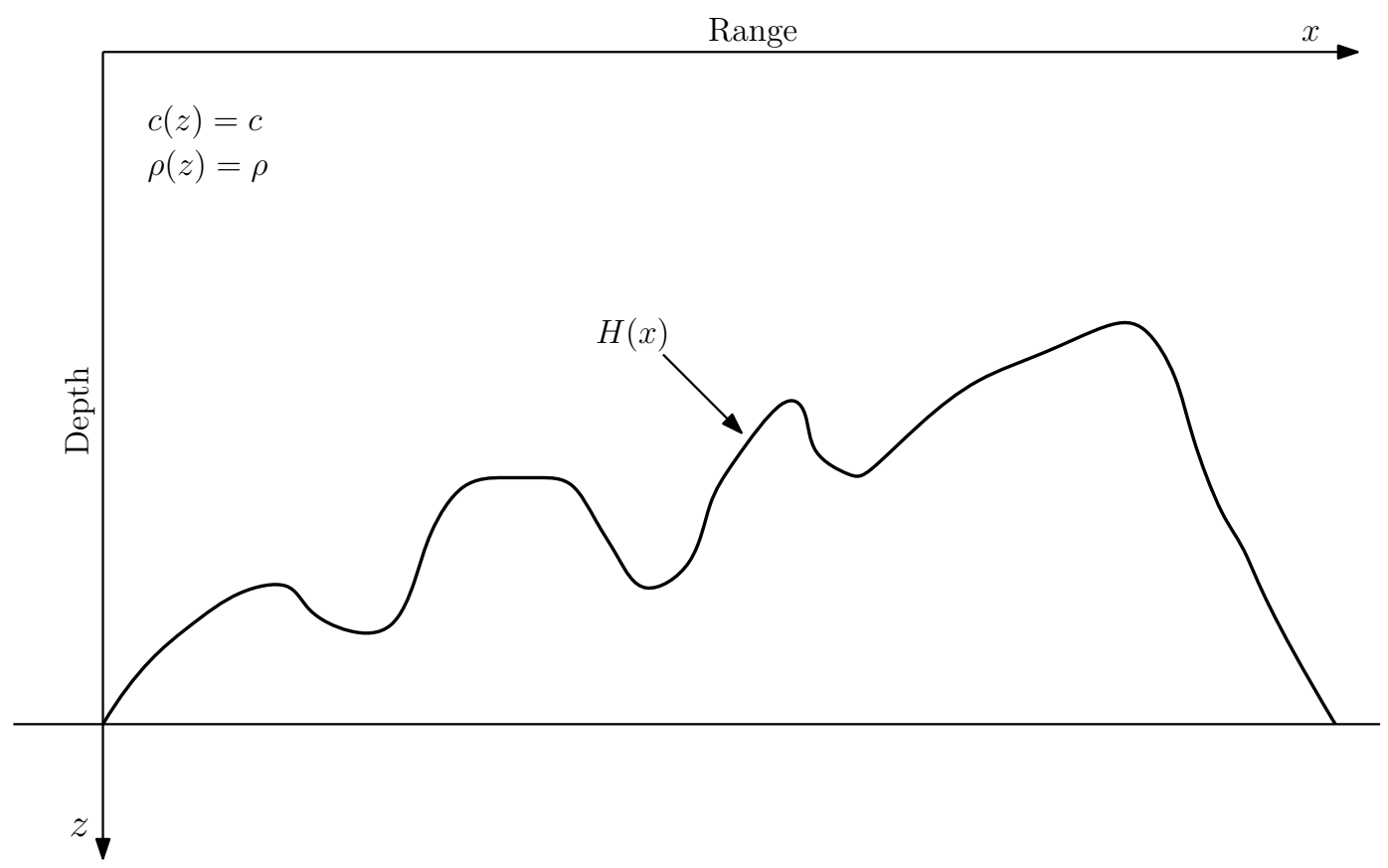

(a)

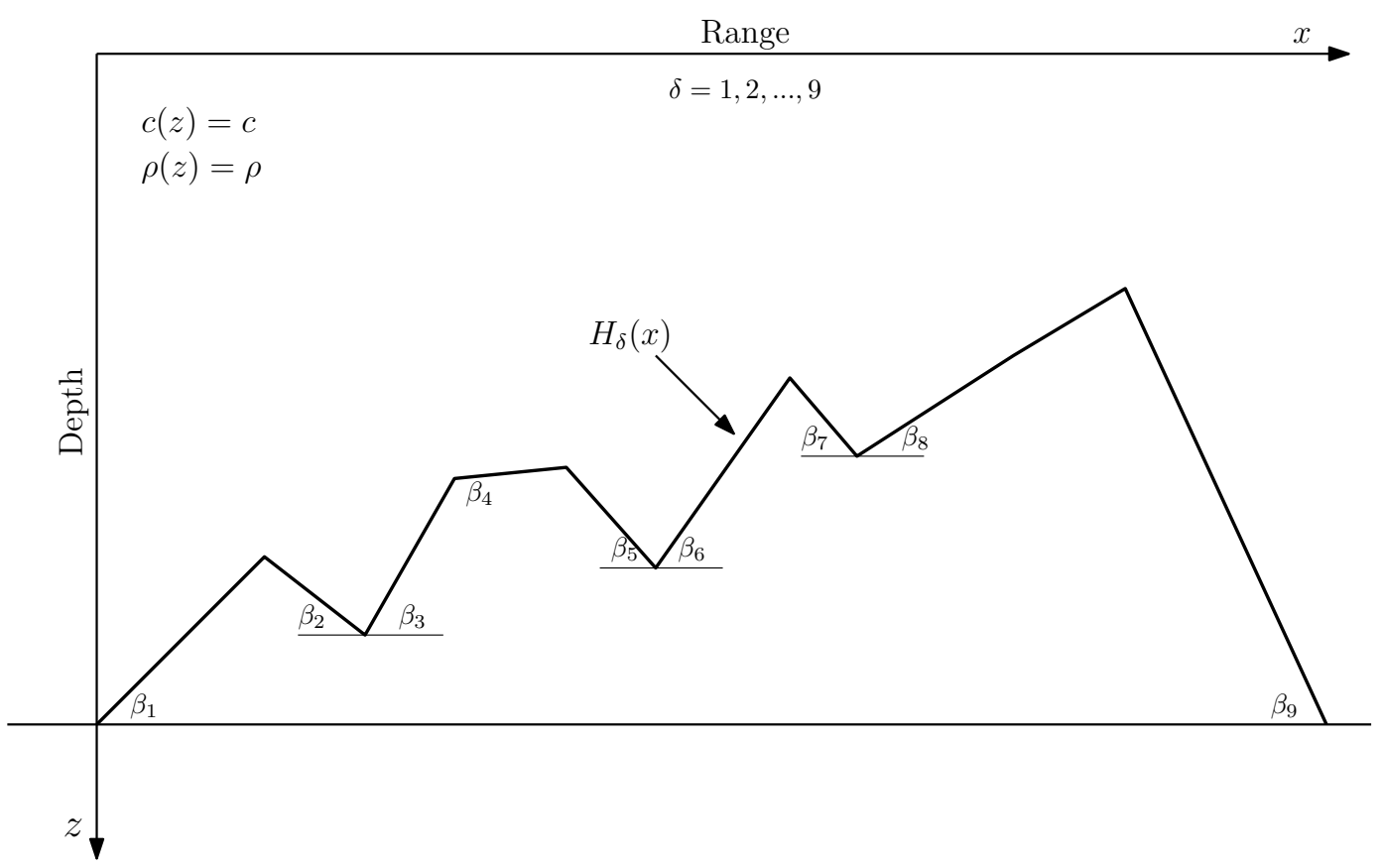

(b)

Figure 3.1: (a) Environment with general range-dependent bottom depth (b) Approximation of the environment in (a) via a series of connected wedge subenvironments. Each subenvironment is defined by the parameter $\beta_{\delta}$, its bottom slope angle. Eigenfunctions and eigenvalues for each subenvironment are given in closedform by Equations (3.17) and (3.18). 
ure(3.1)) occupy the range interval $\left[\alpha_{1}, \alpha_{2}\right]$. We then subdivide this interval into a number of subintervals defined by the boundary points $x=x_{\nu}, \nu=0,1,2, \ldots, \tau$ and consider a general range-dependent function $f_{n}(x)$ that describes the environment and is defined on this range. Next, we shall approximate the function $f_{n}(x)$ by a series of step functions $f_{n \nu}=f_{n}\left(x_{\nu}\right)$ as depicted in Figure (3.2). The range subintervals corresponding to constant values of the function $f_{n}(x)$ will henceforth be referred to as regions. Note that it is always possible to relate the value of $f_{n}$

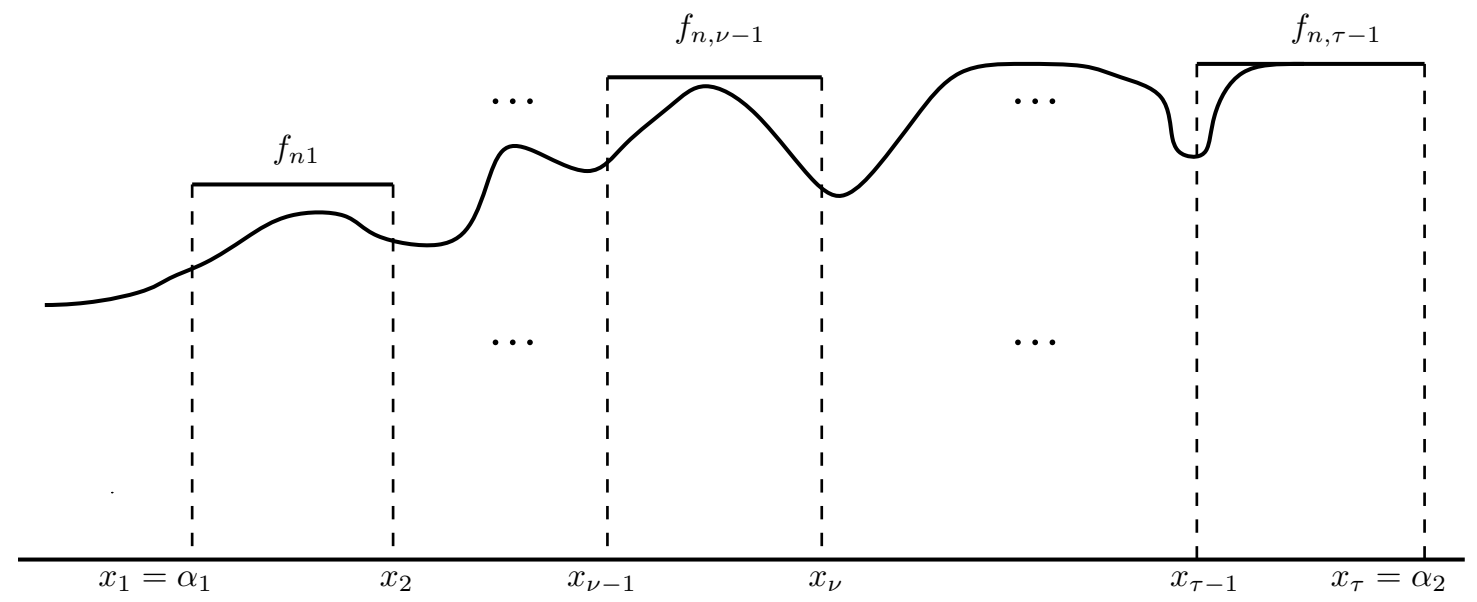

Figure 3.2: Stepwise discretization of subenvironment

in one region to that of an adjacent region via an expansion in range, i.e.:

$$
f_{n, \nu+1}=f_{n \nu}+f_{n \nu}^{\prime} \Delta x+\frac{1}{2} f_{n \nu}^{\prime \prime}(\Delta x)^{2}+\ldots
$$

To illustrate the method we consider the case of two propagating modes and note that the results can be generalized to $N$ modes. We will denote the coefficients of reflection at the interface located at $x=x_{\nu}$ by $A_{n, \nu-1}$ and the coefficients of transmission by $B_{n \nu}$, where $n=1,2$ is the mode index. Given this notation, the incident potential function at a subinterval interface $x=x_{\nu}$ is then:

$$
\hat{\Phi}_{\nu-1}(x, z)=B_{1, \nu-1} e^{i k_{1, \nu-1} x} u_{1, \nu-1}(z)+B_{2, \nu-1} e^{i k_{2, \nu-1} x} u_{2, \nu-1}(z)
$$


This function will give rise to a transmitted set of waves $\hat{\Phi}_{\nu}(x, z)$ in the region $x_{\nu} \leq x<x_{\nu+1}$, and a reflected set, $\hat{\Phi}_{\nu-1}^{*}(x, z)$, for $x<x_{\nu}$ traveling to the left:

$$
\begin{gathered}
\hat{\Phi}_{\nu}(x, z)=B_{1 \nu} e^{i k_{1 \nu} x} u_{1 \nu}(z)+B_{2 \nu} e^{i k_{2 \nu} x} u_{2 \nu}(z) \\
\hat{\Phi}_{\nu-1}^{*}(x, z)=A_{1, \nu-1} e^{-i k_{1, \nu-1} x} u_{1, \nu-1}(z)+A_{2, \nu-1} e^{-i k_{2, \nu-1} x} u_{2, \nu-1}(z) .
\end{gathered}
$$

Note, to simplify the discussion it has been assumed that the first region of interest is sufficiently far away from the source of the acoustic excitation such that the wavefronts are planar. The constants $B_{n \nu}$ and $A_{n \nu}$ of our incident, reflected, and transmitted waves are to be determined from the following continuity conditions:

$$
\begin{aligned}
& \hat{\Phi}_{\nu-1}\left(x_{\nu}, z\right)+\hat{\Phi}_{\nu-1}^{*}\left(x_{\nu}, z\right)=\hat{\Phi}_{\nu}\left(x_{\nu}, z\right) \\
& \hat{\Phi}_{\nu-1}^{\prime}\left(x_{\nu}, z\right)+\hat{\Phi}_{\nu-1}^{* \prime}\left(x_{\nu}, z\right)=\hat{\Phi}_{\nu}^{\prime}\left(x_{\nu}, z\right),
\end{aligned}
$$

where Equation (3.31) is continuity of the total potential and Equation (3.32) is continuity of the normal derivative, each at the interface located at $x=x_{\nu}$. Figure (3.3) depicts this stepwise discretization approach, including an illustration of energy transport through the discretized waveguide. Recalling Equation (3.6),

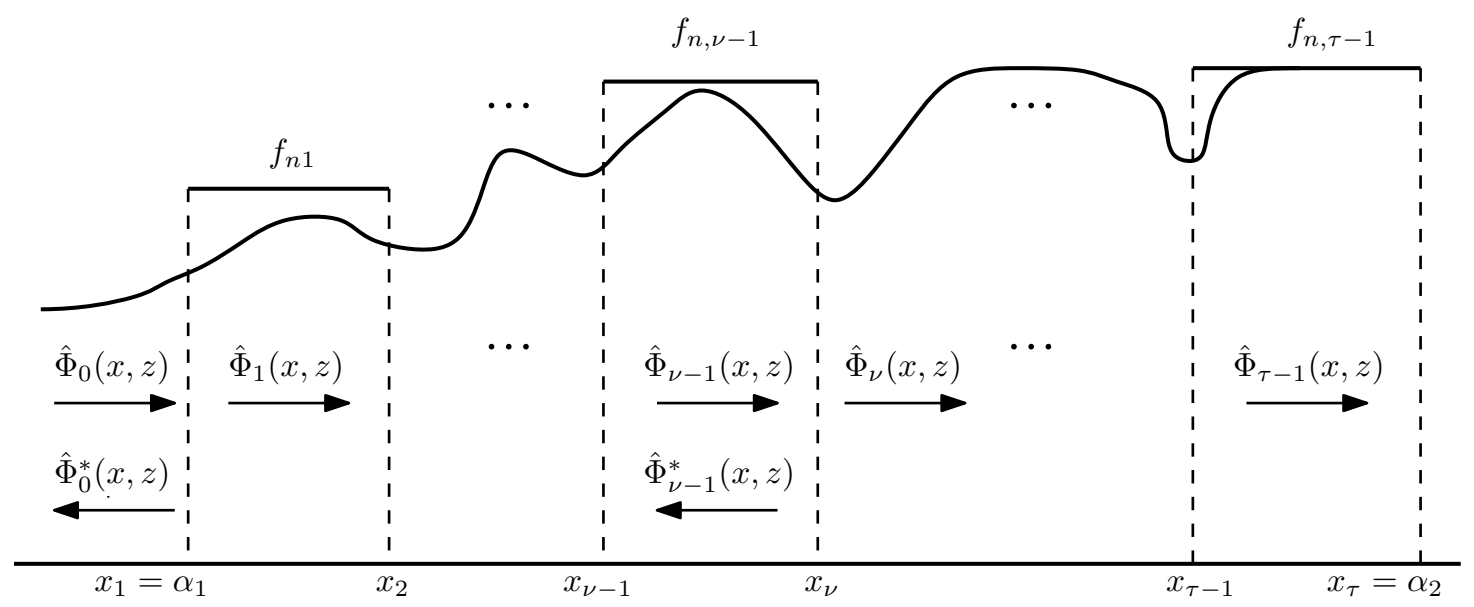

Figure 3.3: Energy transport through the discretized environment

we see that the relation between eigenfunctions in subinterval $\nu-1$ and $\nu$ is given 
by:

$$
\begin{aligned}
& u_{1, \nu-1}=\left\langle u_{1, \nu-1}, u_{1 \nu}\right\rangle u_{1 \nu}+\left\langle u_{1, \nu-1}, u_{2 \nu}\right\rangle u_{2 \nu} \\
& u_{2, \nu-1}=\left\langle u_{2, \nu-1}, u_{1 \nu}\right\rangle u_{1 \nu}+\left\langle u_{2, \nu-1}, u_{2 \nu}\right\rangle u_{2 \nu}
\end{aligned}
$$

Substituting these expressions into the continuity conditions and equating coefficients of $u_{1 \nu}$ and $u_{2 \nu}$ yields a coupled set of difference equations for the coefficients of the reflected and transmitted waves:

$$
\begin{aligned}
B_{1 \nu} e^{i k_{1 \nu} x_{\nu}} & =\left[B_{1, \nu-1} e^{i k_{1, \nu-1} x_{\nu}}+A_{1, \nu-1} e^{-i k_{1, \nu-1} x_{\nu}}\right]\left\langle u_{1, \nu-1}, u_{1 \nu}\right\rangle \\
+ & {\left[B_{2, \nu-1} e^{i k_{2, \nu-1} x_{\nu}}+A_{2, \nu-1} e^{-i k_{2, \nu-1} x_{\nu}}\right]\left\langle u_{2, \nu-1}, u_{1 \nu}\right\rangle } \\
k_{1 \nu} B_{1 \nu} e^{i k_{1 \nu} x_{\nu}} & =k_{1, \nu-1}\left[B_{1, \nu-1} e^{i k_{1, \nu-1} x_{\nu}}-A_{1, \nu-1} e^{-i k_{1, \nu-1} x_{\nu}}\right]\left\langle u_{1, \nu-1}, u_{1 \nu}\right\rangle \\
& +k_{2, \nu-1}\left[B_{2, \nu-1} e^{i k_{2, \nu-1} x_{\nu}}-A_{2, \nu-1} e^{-i k_{2, \nu-1} x_{\nu}}\right]\left\langle u_{2, \nu-1}, u_{1 \nu}\right\rangle \\
B_{2 \nu} e^{i k_{2 \nu} x_{\nu}} & =\left[B_{1, \nu-1} e^{i k_{1, \nu-1} x_{\nu}}+A_{1, \nu-1} e^{-i k_{1, \nu-1} x_{\nu}}\right]\left\langle u_{1, \nu-1}, u_{2 \nu}\right\rangle \\
& +\left[B_{2, \nu-1} e^{i k_{2, \nu-1} x_{\nu}}+A_{2, \nu-1} e^{-i k_{2, \nu-1} x_{\nu}}\right]\left\langle u_{2, \nu-1}, u_{2 \nu}\right\rangle \\
k_{2 \nu} B_{2 \nu} e^{i k_{2 \nu} x_{\nu}} & =k_{1, \nu-1}\left[B_{1, \nu-1} e^{i k_{1, \nu-1} x_{\nu}}-A_{1, \nu-1} e^{-i k_{1, \nu-1} x_{\nu}}\right]\left\langle u_{1, \nu-1}, u_{2 \nu}\right\rangle \\
& +k_{2, \nu-1}\left[B_{2, \nu-1} e^{i k_{2, \nu-1} x_{\nu}}-A_{2, \nu-1} e^{-i k_{2, \nu-1} x_{\nu}}\right]\left\langle u_{2, \nu-1}, u_{2 \nu}\right\rangle .
\end{aligned}
$$

Here, Equations (3.35) and (3.36) were obtained by equating the coefficients of $u_{1 \nu}$, while Equations (3.37) and (3.38) were obtained by equating the coefficients of $u_{2 \nu}$.

The coupled system consisting of Equations (3.35)-(3.38) provides a path toward a solution for energy transport through the discretized range-dependent acoustic environment for which closed-form expressions for the range-expanded normal mode inner product may be used to compute mode coupling. This system is, however, complicated and does not provide a direct means for implementing the desired solution into a computational model for acoustic propagation prediction. Fortunately, simplification of this system of equations is possible by applying the 
previously derived properties of the range-expanded normal mode inner product and making use of a set of asymptotic identities to be developed in the next section.

\subsection{Asymptotic Identities}

Recalling the range-expansion representation in Equations (3.4) and (3.5), a set of asymptotic identities relating the eigenvalues and eigenfunctions in adjacent regions in the discretized medium may be developed. To derive these identities we let $k_{n, \nu-1}$ and $u_{n, \nu-1}$ correspond to the eigenvalue and eigenfunction associated with mode $n$ at range $x$, which exists in region $\nu-1$. Similarly, we let $k_{n \nu}$ and $u_{n \nu}$ correspond to the eigenvalue and eigenfunction associated with mode $n$ at range $x+\Delta x$, which exists in region $\nu$. Given this notation, we first consider the relation $k_{n \nu}+k_{n, \nu-1}$. Applying the range expansion in Equations (3.4) to the term $k_{n \nu}$ and dropping terms higher than second order we obtain the expression:

$$
k_{n \nu}+k_{n, \nu-1} \approx 2 k_{n}(x)+k_{n}^{\prime}(x) \Delta x_{\nu}+\frac{1}{2} k_{n}^{\prime \prime}(x)\left(\Delta x_{\nu}\right)^{2}
$$

Following a similar procedure, the relations

$$
k_{n \nu}-k_{n, \nu-1} \approx k_{n}^{\prime}(x) \Delta x_{\nu}+\frac{1}{2} k_{n}^{\prime \prime}(x)\left(\Delta x_{\nu}\right)^{2}
$$

and

$$
k_{n, \nu-1}-k_{n \nu} \approx-k_{n}^{\prime}(x) \Delta x_{\nu}+\frac{1}{2} k_{n}^{\prime \prime}(x)\left(\Delta x_{\nu}\right)^{2}
$$

may also be obtained.

Next, we apply the range expansion in Equation (3.5) to the term $u_{n \nu}$ to obtain the following two expressions for the range-expanded normal mode inner product:

$$
\begin{aligned}
\left\langle u_{n \nu}, u_{m, \nu-1}\right\rangle & \approx\left\langle u_{n}^{\prime}(z, x), u_{m}(z, x)\right\rangle \Delta x_{\nu} \\
& +\frac{1}{2}\left\langle u_{n}^{\prime \prime}(z, x), u_{m}(z, x)\right\rangle\left(\Delta x_{\nu}\right)^{2}, \quad m \neq n
\end{aligned}
$$


and

$$
\begin{aligned}
\left\langle u_{n \nu}, u_{n, \nu-1}\right\rangle & \approx 1+\left\langle u_{n}^{\prime}(z, x), u_{n}(z, x)\right\rangle \Delta x_{\nu} \\
& +\frac{1}{2}\left\langle u_{n}^{\prime \prime}(z, x), u_{n}(z, x)\right\rangle\left(\Delta x_{\nu}\right)^{2} .
\end{aligned}
$$

Applying the Bachmann-Landau notation developed in Section (1.2), the following asymptotic identities may now be established

$$
\left.\begin{array}{rl}
k_{n \nu}+k_{n, \nu-1} & =\mathcal{O}[1] \\
k_{n \nu}-k_{n, \nu-1} & =\mathcal{O}\left[\Delta x_{\nu}\right] \\
k_{n, \nu-1}-k_{n \nu} & =\mathcal{O}\left[\Delta x_{\nu}\right] \\
\left\langle u_{n \nu}, u_{m, \nu-1}\right\rangle & =\mathcal{O}\left[\Delta x_{\nu}\right] \\
\left\langle u_{n \nu}, u_{n, \nu-1}\right\rangle & =1+\mathcal{O}\left[\Delta x_{\nu}\right]
\end{array}\right\} m \neq n .
$$

These identities will be utilized in simplifying the system of coupled difference equations described by Equations (3.35)-(3.38).

\subsection{Asymptotic Solution}

In deriving a solution to the system in Equations (3.35)-(3.38), we will first derive expressions for the reflection coefficients $A_{1, \nu-1}$ and $A_{2, \nu-1}$ that occur at the interface $x=x_{\nu}$ of our two-mode example. We wish to obtain expressions for these coefficients in terms of the transmission coefficients $B_{1, \nu-1}$ and $B_{2, \nu-1}$ as this will allow for a recursive means of computing the coefficients in any region given knowledge of the initial transmission coefficients in the first region. To solve for the reflection coefficients we begin by multiplying Equation (3.35) by $k_{1 \nu}$ and subtracting Equation (3.36) from the resulting expression. Similarly, we multiply Equation (3.37) by $k_{2 \nu}$ and subtract from it Equation (3.38). These operations 
result in two intermediate equations:

$$
\begin{aligned}
& {\left[A_{1, \nu-1} e^{-i k_{1, \nu-1} x_{\nu}}\left(k_{1 \nu}+k_{1, \nu-1}\right)+B_{1, \nu-1} e^{i k_{1, \nu-1} x_{\nu}}\left(k_{1 \nu}-k_{1, \nu-1}\right)\right]\left\langle u_{1, \nu-1}, u_{1 \nu}\right\rangle} \\
& +\left[A_{2, \nu-1} e^{-i k_{2, \nu-1} x_{\nu}}\left(k_{1 \nu}+k_{2, \nu-1}\right)+B_{2, \nu-1} e^{i k_{2, \nu-1} x_{\nu}}\left(k_{1 \nu}-k_{2, \nu-1}\right)\right]\left\langle u_{2, \nu-1}, u_{1 \nu}\right\rangle \\
& =0 \\
& {\left[A_{1, \nu-1} e^{-i k_{1, \nu-1} x_{\nu}}\left(k_{2 \nu}+k_{1, \nu-1}\right)+B_{1, \nu-1} e^{i k_{1, \nu-1} x_{\nu}}\left(k_{2 \nu}-k_{1, \nu-1}\right)\right]\left\langle u_{1, \nu-1}, u_{2 \nu}\right\rangle} \\
& +\left[A_{2, \nu-1} e^{-i k_{2, \nu-1} x_{\nu}}\left(k_{2 \nu}+k_{2, \nu-1}\right)+B_{2, \nu-1} e^{i k_{2, \nu-1} x_{\nu}}\left(k_{2 \nu}-k_{2, \nu-1}\right)\right]\left\langle u_{2, \nu-1}, u_{2 \nu}\right\rangle \\
& =0 .
\end{aligned}
$$

To solve for $A_{1, \nu-1}$ we eliminate $A_{2, \nu-1}$ from these expressions by multiplying Equation (3.45) by $\left(k_{2 \nu}+k_{2, \nu-1}\right)\left\langle u_{2, \nu-1}, u_{2 \nu}\right\rangle$ and Equation (3.46) by $\left(k_{1 \nu}+k_{2, \nu-1}\right)\left\langle u_{2, \nu-1}, u_{1 \nu}\right\rangle$ and subtracting the two resulting expressions. This leads to the expression:

$$
\begin{aligned}
& A_{1, \nu-1} e^{-i k_{1, \nu-1} x_{\nu}}\left[\left(k_{1 \nu}+k_{1, \nu-1}\right)\left(k_{2 \nu}+k_{2, \nu-1}\right)\left\langle u_{1, \nu-1}, u_{1 \nu}\right\rangle\left\langle u_{2, \nu-1}, u_{2 \nu}\right\rangle\right.\left.-\left(k_{2 \nu}+k_{1, \nu-1}\right)\left(k_{1 \nu}+k_{2, \nu-1}\right)\left\langle u_{1, \nu-1}, u_{2 \nu}\right\rangle\left\langle u_{2, \nu-1}, u_{1 \nu}\right\rangle\right] \\
&+B_{1, \nu-1} e^{i k_{1, \nu-1} x_{\nu}}\left[\left(k_{1 \nu}-k_{1, \nu-1}\right)\left(k_{2 \nu}+k_{2, \nu-1}\right)\left\langle u_{1, \nu-1}, u_{1 \nu}\right\rangle\left\langle u_{2, \nu-1}, u_{2 \nu}\right\rangle\right. \\
&\left.-\left(k_{2 \nu}-k_{1, \nu-1}\right)\left(k_{1 \nu}+k_{2, \nu-1}\right)\left\langle u_{1, \nu-1}, u_{2 \nu}\right\rangle\left\langle u_{2, \nu-1}, u_{1 \nu}\right\rangle\right] \\
&+B_{2, \nu-1} e^{i k_{2, \nu-1} x_{\nu}}\left[\left(k_{1 \nu}-k_{2, \nu-1}\right)\left(k_{2 \nu}+k_{2, \nu-1}\right)\left\langle u_{2, \nu-1}, u_{1 \nu}\right\rangle\left\langle u_{2, \nu-1}, u_{2 \nu}\right\rangle\right. \\
&\left.-\left(k_{2 \nu}-k_{2, \nu-1}\right)\left(k_{1 \nu}+k_{2, \nu-1}\right)\left\langle u_{2, \nu-1}, u_{2 \nu}\right\rangle\left\langle u_{2, \nu-1}, u_{1 \nu}\right\rangle\right] \\
&=0 .
\end{aligned}
$$

To simplify Equation (3.47) we apply the asymptotic identities given in Equation (3.44) and recall that $\Delta x_{\nu}$ is small and therefore it is reasonable to eliminate terms of $\mathcal{O}\left[(\Delta x)^{2}\right]$ and smaller. In doing so and dividing by $\left(k_{2 \nu}+k_{2, \nu-1}\right)$, we arrive at 
the following simplified equation:

$$
\begin{aligned}
& A_{1, \nu-1} e^{-i k_{1, \nu-1} x_{\nu}}\left(k_{1 \nu}+k_{1, \nu-1}\right)\left\langle u_{1, \nu-1}, u_{1 \nu}\right\rangle\left\langle u_{2, \nu-1}, u_{2 \nu}\right\rangle \\
& +B_{1, \nu-1} e^{i k_{1, \nu-1} x_{\nu}}\left(k_{1 \nu}-k_{1, \nu-1}\right)\left\langle u_{1, \nu-1}, u_{1 \nu}\right\rangle\left\langle u_{2, \nu-1}, u_{2 \nu}\right\rangle \\
& +B_{2, \nu-1} e^{i k_{2, \nu-1} x_{\nu}}\left(k_{1 \nu}-k_{2, \nu-1}\right)\left\langle u_{2, \nu-1}, u_{1 \nu}\right\rangle\left\langle u_{2, \nu-1}, u_{2 \nu}\right\rangle=0 .
\end{aligned}
$$

Next, we recognize that

$$
\begin{aligned}
k_{1 \nu}+k_{1, \nu-1} & =2 k_{1, \nu-1}+\left(k_{1 \nu}-k_{1, \nu-1}\right) \\
& =2 k_{1, \nu-1}+\mathcal{O}\left[\Delta x_{\nu}\right]
\end{aligned}
$$

and

$$
\begin{aligned}
k_{1 \nu}-k_{2, \nu-1} & =\left(k_{1, \nu-1}-k_{2, \nu-1}\right)+\left(k_{1 \nu}-k_{1, \nu-1}\right) \\
& =\left(k_{1, \nu-1}-k_{2, \nu-1}\right)+\mathcal{O}\left[\Delta x_{\nu}\right] .
\end{aligned}
$$

If we now substitute into Equation (3.48) the expressions on the right side of Equations (3.49) and (3.50) and again drop terms of $\mathcal{O}\left[(\Delta x)^{2}\right]$ and smaller from the resulting expression we arrive at the following asymptotic solution for the reflection coefficient $A_{1, \nu-1}$ :

$$
\begin{aligned}
A_{1, \nu-1}= & -B_{1, \nu-1} \frac{\left(k_{1 \nu}-k_{1, \nu-1}\right)}{2 k_{1, \nu-1}} e^{2 i k_{1, \nu-1} x_{\nu}} \\
& -B_{2, \nu-1} \frac{\left(k_{1, \nu-1}-k_{2, \nu-1}\right)}{2 k_{1, \nu-1}} e^{i\left(k_{1, \nu-1}+k_{2, \nu-1}\right) x_{\nu}} \frac{\left\langle u_{2, \nu-1}, u_{1 \nu}\right\rangle}{\left\langle u_{1, \nu-1}, u_{1 \nu}\right\rangle} .
\end{aligned}
$$

To solve for $A_{2, \nu-1}$ we follow a similar procedure. We eliminate $A_{1, \nu-1}$ from Equations (3.45) and (3.46) by multiplying Equation (3.45) by $\left(k_{2 \nu}+k_{1, \nu-1}\right)\left\langle u_{1, \nu-1}, u_{2 \nu}\right\rangle$ and Equation (3.46) by $\left(k_{1 \nu}+k_{1, \nu-1}\right)\left\langle u_{1, \nu-1}, u_{1 \nu}\right\rangle$ and 
subtracting the two resulting expressions. This leads to:

$$
\begin{aligned}
& A_{2, \nu-1} e^{-i k_{2, \nu-1} x_{\nu}}\left[\left(k_{1 \nu}+k_{2, \nu-1}\right)\left(k_{2 \nu}+k_{1, \nu-1}\right)\left\langle u_{2, \nu-1}, u_{1 \nu}\right\rangle\left\langle u_{1, \nu-1}, u_{2 \nu}\right\rangle\right.\left.-\left(k_{2 \nu}+k_{2, \nu-1}\right)\left(k_{1 \nu}+k_{1, \nu-1}\right)\left\langle u_{2, \nu-1}, u_{2 \nu}\right\rangle\left\langle u_{1, \nu-1}, u_{1 \nu}\right\rangle\right] \\
&+B_{1, \nu-1} e^{i k_{1, \nu-1} x_{\nu}}\left[\left(k_{1 \nu}-k_{1, \nu-1}\right)\left(k_{2 \nu}+k_{1, \nu-1}\right)\left\langle u_{1, \nu-1}, u_{1 \nu}\right\rangle\left\langle u_{1, \nu-1}, u_{2 \nu}\right\rangle\right. \\
&\left.-\left(k_{2 \nu}-k_{1, \nu-1}\right)\left(k_{1 \nu}+k_{1, \nu-1}\right)\left\langle u_{1, \nu-1}, u_{2 \nu}\right\rangle\left\langle u_{1, \nu-1}, u_{1 \nu}\right\rangle\right] \\
&+B_{2, \nu-1} e^{i k_{2, \nu-1} x_{\nu}}\left[\left(k_{1 \nu}-k_{2, \nu-1}\right)\left(k_{2 \nu}+k_{1, \nu-1}\right)\left\langle u_{2, \nu-1}, u_{1 \nu}\right\rangle\left\langle u_{1, \nu-1}, u_{2 \nu}\right\rangle\right. \\
&\left.-\left(k_{2 \nu}-k_{2, \nu-1}\right)\left(k_{1 \nu}+k_{1, \nu-1}\right)\left\langle u_{2, \nu-1}, u_{2 \nu}\right\rangle\left\langle u_{1, \nu-1}, u_{1 \nu}\right\rangle\right] \\
&=0 .
\end{aligned}
$$

Once again, we utilize the asymptotic identities given in Equation (3.44) to simplify the above expression by eliminating terms of $\mathcal{O}\left[(\Delta x)^{2}\right]$ and smaller. After dividing by $\left(k_{1 \nu}+k_{1, \nu-1}\right)$, this simplification leads to the expression

$$
\begin{aligned}
& -A_{2, \nu-1} e^{-i k_{2, \nu-1} x_{\nu}}\left(k_{2 \nu}+k_{2, \nu-1}\right)\left\langle u_{2, \nu-1}, u_{2 \nu}\right\rangle\left\langle u_{1, \nu-1}, u_{1 \nu}\right\rangle \\
& -B_{1, \nu-1} e^{i k_{1, \nu-1} x_{\nu}}\left(k_{2 \nu}-k_{1, \nu-1}\right)\left\langle u_{1, \nu-1}, u_{2 \nu}\right\rangle\left\langle u_{1, \nu-1}, u_{1 \nu}\right\rangle \\
& -B_{2, \nu-1} e^{i k_{2, \nu-1} x_{\nu}}\left(k_{2 \nu}-k_{2, \nu-1}\right)\left\langle u_{2, \nu-1}, u_{2 \nu}\right\rangle\left\langle u_{1, \nu-1}, u_{1 \nu}\right\rangle=0 .
\end{aligned}
$$

Using similar arguments as those used in Equations (3.49) and (3.50) we can replace $k_{2 \nu}+k_{2, \nu-1}$ in Equation (3.53) with $2 k_{2, \nu-1}+\mathcal{O}\left[\Delta x_{\nu}\right]$ and $k_{2 \nu}-k_{1, \nu-1}$ with $\left(k_{2, \nu-1}-k_{1, \nu-1}\right)+\mathcal{O}\left[\Delta x_{\nu}\right]$. Eliminating any remaining terms that are smaller than $\mathcal{O}[\Delta x]$ leads to the following asymptotic solution for $A_{2, \nu-1}$ :

$$
\begin{aligned}
A_{2, \nu-1}= & -B_{1, \nu-1} \frac{\left(k_{2, \nu-1}-k_{1, \nu-1}\right)}{2 k_{2, \nu-1}} e^{i\left(k_{1, \nu-1}+k_{2, \nu-1}\right) x_{\nu}} \frac{\left\langle u_{1, \nu-1}, u_{2 \nu}\right\rangle}{\left\langle u_{2, \nu-1}, u_{2 \nu}\right\rangle} \\
& -B_{2, \nu-1} \frac{\left(k_{2 \nu}-k_{2, \nu-1}\right)}{2 k_{2, \nu-1}} e^{2 i k_{2, \nu-1} x_{\nu}} .
\end{aligned}
$$

To derive expressions for the transmission coefficients $B_{1 \nu}$ and $B_{2 \nu}$ for our two mode example in terms of the coefficients $B_{1, \nu-1}$ and $B_{2, \nu-1}$ we follow a procedure 
similar to that for the reflection coefficients. First we multiply Equation (3.35) by $k_{1, \nu-1}$ and add Equation (3.36) to the resulting expression. Similarly, we multiply Equation (3.37) by $k_{2, \nu-1}$ and add to it Equation (3.38). Doing so, we arrive at the intermediate equations:

$$
\begin{aligned}
B_{1 \nu} e^{i k_{1 \nu} x_{\nu}}\left(k_{1, \nu-1}+k_{1 \nu}\right) & =2 k_{1, \nu-1} B_{1, \nu-1} e^{i k_{1, \nu-1} x_{\nu}}\left\langle u_{1, \nu-1}, u_{1 \nu}\right\rangle \\
& +\left[A_{2, \nu-1} e^{-i k_{2, \nu-1} x_{\nu}}\left(k_{1, \nu-1}-k_{2, \nu-1}\right)\right. \\
& \left.+B_{2, \nu-1} e^{i k_{2, \nu-1} x_{\nu}}\left(k_{1, \nu-1}+k_{2, \nu-1}\right)\right]\left\langle u_{2, \nu-1}, u_{1 \nu}\right\rangle
\end{aligned}
$$

and

$$
\begin{aligned}
B_{2 \nu} e^{i k_{2 \nu} x_{\nu}}\left(k_{2, \nu-1}+k_{2 \nu}\right) & =\left[B_{1, \nu-1} e^{i k_{1, \nu-1} x_{\nu}}\left(k_{2, \nu-1}+k_{1, \nu-1}\right)\right. \\
& \left.+A_{1, \nu-1} e^{-i k_{1, \nu-1} x_{\nu}}\left(k_{2, \nu-1}-k_{1, \nu-1}\right)\right]\left\langle u_{1, \nu-1}, u_{2 \nu}\right\rangle \\
& +2 k_{2, \nu-1} B_{2, \nu-1} e^{i k_{2, \nu-1} x_{\nu}}\left\langle u_{2, \nu-1}, u_{2 \nu}\right\rangle .
\end{aligned}
$$

Next we substitute the known values of $A_{1, \nu-1}$ and $A_{2, \nu-1}$ from Equations (3.51) and (3.54) into Equations (3.55) and (3.56). Doing so and solving Equations (3.55) for $B_{1 \nu}$ we arrive at the expression:

$$
\begin{aligned}
& B_{1 \nu}=B_{1, \nu-1} \frac{e^{i k_{1, \nu-1} x_{\nu}}}{e^{i k_{1 \nu} x_{\nu}}\left(k_{1, \nu-1}+k_{1 \nu}\right)} \\
& \times\left[2 k_{1, \nu-1}\left\langle u_{1, \nu-1}, u_{1 \nu}\right\rangle\right. \\
& \left.\quad-\frac{\left(k_{2, \nu-1}-k_{1, \nu-1}\right)\left(k_{1, \nu-1}-k_{2, \nu-1}\right)}{2 k_{2, \nu-1}} \frac{\left\langle u_{1, \nu-1}, u_{2 \nu}\right\rangle}{\left\langle u_{2, \nu-1}, u_{2 \nu}\right\rangle}\left\langle u_{2, \nu-1}, u_{1 \nu}\right\rangle\right] \\
& \left.+B_{2, \nu-1} \frac{e^{i k_{2, \nu-1} x_{\nu}}}{e^{i k_{1 \nu} x_{\nu}\left(k_{1, \nu-1}+k_{1 \nu}\right)}}\right]\left\langle u_{2, \nu-1}, u_{1 \nu}\right\rangle .
\end{aligned}
$$

We simplify Equation (3.57) by performing an order of magnitude analysis of its terms using the asymptotic identities given in Equation (3.44) and eliminating terms of $\mathcal{O}\left[(\Delta x)^{2}\right]$ and smaller. This simplification yields the following asymptotic 
solution for $B_{1 \nu}$ :

$$
\begin{aligned}
B_{1 \nu}= & B_{1, \nu-1} \frac{2 k_{1, \nu-1}}{\left(k_{1, \nu-1}+k_{1 \nu}\right)} e^{i\left(k_{1, \nu-1}-k_{1 \nu}\right) x_{\nu}}\left\langle u_{1, \nu-1}, u_{1 \nu}\right\rangle \\
& +B_{2, \nu-1} \frac{\left(k_{2, \nu-1}+k_{1, \nu-1}\right)}{2 k_{1, \nu-1}} e^{i\left(k_{2, \nu-1}-k_{1 \nu}\right) x_{\nu}}\left\langle u_{2, \nu-1}, u_{1 \nu}\right\rangle .
\end{aligned}
$$

To obtain a solution for $B_{2 \nu}$ we follow a similar procedure. We begin by solving Equation (3.56) for $B_{2 \nu}$, where $A_{1, \nu-1}$ and $A_{2, \nu-1}$ and are replaced with their known values. This results in the expression:

$$
\begin{aligned}
& B_{2 \nu}=B_{1, \nu-1} \frac{e^{i k_{1, \nu-1} x_{\nu}}}{e^{i k_{2 \nu} x_{\nu}}\left(k_{2, \nu-1}+k_{2 \nu}\right)} \\
& \times\left[\left(k_{2, \nu-1}+k_{1, \nu-1}\right)-\frac{\left(k_{1 \nu}-k_{1, \nu-1}\right)\left(k_{2, \nu-1}-k_{1, \nu-1}\right)}{2 k_{1, \nu-1}}\right]\left\langle u_{1, \nu-1}, u_{2 \nu}\right\rangle \\
& +B_{2, \nu-1} \frac{e^{i k_{2, \nu-1} x_{\nu}}}{e^{i k_{2 \nu} x_{\nu}}\left(k_{2, \nu-1}+k_{2 \nu}\right)} \\
& \times\left[2 k_{2, \nu-1}\left\langle u_{2, \nu-1}, u_{2 \nu}\right\rangle\right. \\
& \left.\quad-\frac{\left(k_{1, \nu-1}-k_{2, \nu-1}\right)\left(k_{2, \nu-1}-k_{1, \nu-1}\right)}{2 k_{1, \nu-1}} \frac{\left\langle u_{2, \nu-1}, u_{1 \nu}\right\rangle}{\left\langle u_{1, \nu-1}, u_{1 \nu}\right\rangle}\left\langle u_{1, \nu-1}, u_{2 \nu}\right\rangle\right] .
\end{aligned}
$$

Yet again utilizing the asymptotic identities from Equation (3.44), we eliminate terms of $\mathcal{O}\left[(\Delta x)^{2}\right]$ and smaller and arrive at the following asymptotic solution for $B_{2 \nu}$ :

$$
\begin{aligned}
B_{2 \nu}= & B_{1, \nu-1} \frac{\left(k_{1, \nu-1}+k_{2, \nu-1}\right)}{2 k_{2, \nu-1}} e^{i\left(k_{1, \nu-1}-k_{2 \nu}\right) x_{\nu}}\left\langle u_{1, \nu-1}, u_{2 \nu}\right\rangle \\
& +B_{2, \nu-1} \frac{2 k_{2, \nu-1}}{\left(k_{2, \nu-1}+k_{2 \nu}\right)} e^{i\left(k_{2, \nu-1}-k_{2 \nu}\right) x_{\nu}}\left\langle u_{2, \nu-1}, u_{2 \nu}\right\rangle
\end{aligned}
$$

The asymptotic expressions for the reflection and transmission coefficients admit a convenient matrix formulation. To express the matrix form of these solutions we begin by defining a reflection coefficient matrix, $R_{\nu}$, and a transmission coefficient matrix, $T_{\nu}$ :

$$
R_{\nu}=\left(\begin{array}{ll}
\kappa_{11}^{R_{\nu}} e^{2 i k_{1, \nu-1} x_{\nu}} & \kappa_{12}^{R_{\nu}} e^{i\left(k_{1, \nu-1}+k_{2, \nu-1}\right) x_{\nu}} \frac{\left\langle u_{2, \nu-1}, u_{1 \nu}\right\rangle}{\left\langle u_{1, \nu-1}, u_{1 \nu}\right\rangle} \\
\kappa_{21}^{R_{\nu}} e^{i\left(k_{1, \nu-1}+k_{2, \nu-1}\right) x_{\nu}} \frac{\left\langle u_{1, \nu-1}, u_{2 \nu}\right\rangle}{\left\langle u_{2, \nu-1}, u_{2 \nu}\right\rangle} & \kappa_{22}^{R_{\nu}} e^{2 i k_{2, \nu-1} x_{\nu}}
\end{array}\right),
$$


where

$$
\kappa_{m n}^{R_{\nu}}= \begin{cases}\frac{-\left(k_{m \nu}-k_{m, \nu-1}\right)}{2 k_{m, \nu-1}} & m=n \\ \frac{-\left(k_{m, \nu-1}-k_{n, \nu-1}\right)}{2 k_{m, \nu-1}} & m \neq n\end{cases}
$$

and

$$
T_{\nu}=\left(\begin{array}{cc}
\kappa_{11}^{T_{\nu}} e^{i\left(k_{1, \nu-1}-k_{1 \nu}\right) x_{\nu}}\left\langle u_{1, \nu-1}, u_{1 \nu}\right\rangle & \kappa_{12}^{T_{\nu}} e^{i\left(k_{2, \nu-1}-k_{1 \nu}\right) x_{\nu}}\left\langle u_{2, \nu-1}, u_{1 \nu}\right\rangle \\
\kappa_{21}^{T_{\nu}} e^{i\left(k_{1, \nu-1}-k_{2 \nu}\right) x_{\nu}}\left\langle u_{1, \nu-1}, u_{2 \nu}\right\rangle & \kappa_{22}^{T_{\nu}} e^{i\left(k_{2, \nu-1}-k_{2 \nu}\right) x_{\nu}}\left\langle u_{2, \nu-1}, u_{2 \nu}\right\rangle
\end{array}\right),
$$

where

$$
\kappa_{m n}^{T_{\nu}}= \begin{cases}\frac{2 k_{m, \nu-1}}{\left(k_{m, \nu-1}+k_{m \nu}\right)} & m=n \\ \frac{\left(k_{m, \nu-1}+k_{n, \nu-1}\right)}{2 k_{m, \nu-1}} & m \neq n\end{cases}
$$

This allows one to write the following recursive matrix expressions for the reflection and transmission coefficients:

$$
\left(\begin{array}{l}
A_{1, \nu-1} \\
A_{2, \nu-1}
\end{array}\right)=R_{\nu}\left(\begin{array}{l}
B_{1, \nu-1} \\
B_{2, \nu-1}
\end{array}\right)=R_{\nu} \prod_{\sigma=1}^{\nu-1} T_{\sigma}\left(\begin{array}{l}
B_{10} \\
B_{20}
\end{array}\right)
$$

and

$$
\left(\begin{array}{l}
B_{1 \nu} \\
B_{2 \nu}
\end{array}\right)=T_{\nu}\left(\begin{array}{l}
B_{1, \nu-1} \\
B_{2, \nu-1}
\end{array}\right)=\prod_{\sigma=1}^{\nu} T_{\sigma}\left(\begin{array}{l}
B_{10} \\
B_{20}
\end{array}\right),
$$

where $B_{10}$ and $B_{20}$ are the transmission coefficients corresponding to modes 1 and 2 , respectively, in the region containing the acoustic source. If acoustic absorption is considered negligible in this region both of these coefficients are equal to unity.

The recursive formulae in Equations (3.65) and (3.66) represent the principle result of the presented theory. These expressions provide a means of mathematically modeling energy transport through a range-dependent shallow water acoustic medium where non-adiabatic coupling between modes is estimated via the rangeexpanded normal mode inner product discussed in Section (3.2). Moreover, this 
difference equations solution is easily implemented into a discrete computing device thus making it suitable for approximating non-adiabatic mode coupling in a computational model for acoustic propagation prediction. When paired with the discretization approach discussed in Section (3.3), this solution has the potential to provide significant computational savings over currently existing coupled mode solutions. In particular, the ability to compute mode coupling via closed-form algebraic expressions offers significant savings as it avoids the need for numerically solving an inner product integral at each of the interfaces separating the horizontal regions throughout the medium. The elimination of the eigenvalue search problem is an additional benefit of this asymptotic coupled mode solution, as mentioned previously.

The remaining goal is to extend this solution to study a particular problem of interest: mode coupling in shallow water due to horizontally-variable bottom depth. To accomplish this we will first adapt the solution to allow for non-planar wavefronts due to short range (i.e. near-source) propagation. This will allow for the formulation of a transmission loss equation for propagation in a cylindrical waveguide. Following this, adjustments will be made to the solution to ensure numerical stability. Finally, transmission loss estimates produced via this asymptotic stepwise coupled mode solution will be compared to benchmark solutions for several cases of interest.

\subsection{Cylindrical Wave Extension}

In many propagation scenarios of interest the plane wave assumption made in Section (3.3) is insufficient. This is due to the fact that at short distances from the acoustic source the wavefronts of an omnidirectional point source are typically spherical or cylindrical depending on the nature of the waveguide. For the case of upslope propagation in shallow water this is particularly true as the constant 
decrease in waveguide depth causes the modes of vibration of the acoustic medium to become rapidly cutoff, restricting the propagation scenario to shorter ranges. To extend the solution in Section (3.5) to these scenarios one must reformulate the expressions for the incident, reflected, and transmitted waves in Equations (3.28)-(3.30) to accommodate non-planar wavefronts.

Going forward, we will assume that acoustic propagation is occurring in a shallow water cylindrical waveguide with azimuthal symmetry. The wave equation governing the propagation of acoustic energy in this scenario was discussed in Section (2.2), and the coupled equation governing energy transport with range was discussed in Section (2.6). Applying the discretization technique of Section (3.3) to this waveguide, the incident potential function representing the set of waves arriving at an interface located at range $r=r_{\nu}$ due to an acoustic excitation created by a source located at $r=0$ is given by the expression

$$
\begin{aligned}
\hat{\Phi}_{\nu-1}(r, z)=\frac{i \dot{m}}{\sqrt{8 \pi r} \rho(z)} e^{-i \pi / 4}\{ & B_{1, \nu-1} e^{i k_{1, \nu-1} r} \frac{u_{1, \nu-1}\left(z_{s}\right)}{\rho\left(z_{s}\right) \sqrt{k_{1, \nu-1}}} u_{1, \nu-1}(z) \\
& \left.+B_{2, \nu-1} e^{i k_{2, \nu-1} r} \frac{u_{2, \nu-1}\left(z_{s}\right)}{\rho\left(z_{s}\right) \sqrt{k_{2, \nu-1}}} u_{2, \nu-1}(z)\right\},
\end{aligned}
$$

where the asymptotic solution in Equation (2.25) of Section (2.2) has been used to inform the expression in Equation (3.67). As was the case for the original derivation, we will limit ourselves to two modes to simplify the discussion but note that the method is, again, generalizable to $N$ modes. As in the plane wave example, the incident set of waves given by Equation (3.67) will give rise to a set of transmitted waves $\hat{\Phi}_{\nu}$ in the region $r_{\nu} \leq r<r_{\nu+1}$, and a reflected set, $\hat{\Phi}_{\nu-1}^{*}$, for $r<r_{\nu}$ traveling to the left. These waves are described by the expressions

$$
\begin{aligned}
\hat{\Phi}_{\nu}(r, z)=\frac{i \dot{m}}{\sqrt{8 \pi r} \rho(z)} e^{-i \pi / 4}\{ & B_{1 \nu} e^{i k_{1 \nu} r} \frac{u_{1 \nu}\left(z_{s}\right)}{\rho\left(z_{s}\right) \sqrt{k_{1 \nu}}} u_{1 \nu}(z) \\
& \left.+B_{2 \nu} e^{i k_{2 \nu} r} \frac{u_{2 \nu}\left(z_{s}\right)}{\rho\left(z_{s}\right) \sqrt{k_{2 \nu}}} u_{2 \nu}(z)\right\}
\end{aligned}
$$


and

$$
\left.\begin{array}{rl}
\hat{\Phi}_{\nu-1}^{*}(r, z)=-\frac{i \dot{m}}{\sqrt{8 \pi r} \rho(z)} e^{i \pi / 4}\{ & A_{1, \nu-1} e^{-i k_{1, \nu-1} r} \frac{u_{1, \nu-1}\left(z_{s}\right)}{\rho\left(z_{s}\right) \sqrt{k_{1, \nu-1}}} u_{1, \nu-1}(z) \\
& +A_{2, \nu-1} e^{-i k_{2, \nu-1} r} \frac{u_{2, \nu-1}\left(z_{s}\right)}{\rho\left(z_{s}\right) \sqrt{k_{2, \nu-1}}} u_{2, \nu-1}(z)
\end{array}\right\}
$$

As was the case in Section (3.3), application of the continuity conditions given by Equations (3.31) and (3.32) along with the relationship given by Equations (3.33) and (3.34) leads to a coupled set of difference equations for the constants $B_{n \nu}$ and $A_{n \nu}$ of our incident, reflected, and transmitted waves. This system is simplified following a procedure identical to that of Section (3.5), leading to the following reflection and transmission coefficient matrices for our discretized cylindrical waveguide:

$$
R_{\nu}=\left(\begin{array}{ll}
\frac{\kappa_{11}^{R_{\nu}}}{i} e^{2 i k_{1, \nu-1} r_{\nu}} & \frac{\kappa_{12}^{R_{\nu}}}{i} e^{i\left(k_{1, \nu-1}+k_{2, \nu-1}\right) r_{\nu}} \frac{\left\langle u_{2, \nu-1}, u_{1 \nu}\right\rangle}{\left\langle u_{1, \nu-1}, u_{1 \nu}\right\rangle} \gamma_{12}^{R_{\nu}} \\
\frac{\kappa_{21}^{R_{\nu}}}{i} e^{i\left(k_{1, \nu-1}+k_{2, \nu-1}\right) r_{\nu}} \frac{\left\langle u_{1, \nu-1}, u_{2 \nu}\right\rangle}{\left\langle u_{2, \nu-1}, u_{2 \nu}\right\rangle} \gamma_{21}^{R_{\nu}} & \frac{\kappa_{22}^{R_{\nu}}}{i} e^{2 i k_{2, \nu-1} r_{\nu}}
\end{array}\right),
$$

where

$$
\gamma_{m n}^{R_{\nu}}=\frac{u_{n, \nu-1}\left(z_{s}\right)}{u_{m, \nu-1}\left(z_{s}\right)} \frac{\sqrt{k_{m, \nu-1}}}{\sqrt{k_{n, \nu-1}}}, \quad m \neq n
$$

and

$$
T_{\nu}=\left(\begin{array}{cc}
\kappa_{11}^{T_{\nu}} e^{i\left(k_{1, \nu-1}-k_{1 \nu}\right) r_{\nu}}\left\langle u_{1, \nu-1}, u_{1 \nu}\right\rangle \gamma_{11}^{T_{\nu}} & \kappa_{12}^{T_{\nu}} e^{i\left(k_{2, \nu-1}-k_{1 \nu}\right) r_{\nu}}\left\langle u_{2, \nu-1}, u_{1 \nu}\right\rangle \gamma_{12}^{T_{\nu}} \\
\kappa_{21}^{T_{\nu}} e^{i\left(k_{1, \nu-1}-k_{2 \nu}\right) r_{\nu}}\left\langle u_{1, \nu-1}, u_{2 \nu}\right\rangle \gamma_{21}^{T_{\nu}} & \kappa_{22}^{T_{\nu}} e^{i\left(k_{2, \nu-1}-k_{2 \nu}\right) r_{\nu}}\left\langle u_{2, \nu-1}, u_{2 \nu}\right\rangle \gamma_{22}^{T_{\nu}}
\end{array}\right),
$$

where

$$
\gamma_{m n}^{T_{\nu}}= \begin{cases}\frac{u_{m, \nu-1}\left(z_{s}\right)}{u_{m \nu}\left(z_{s}\right)} \frac{\sqrt{k_{m \nu}}}{\sqrt{k_{m, \nu-1}}} & m=n \\ \frac{u_{n, \nu-1}\left(z_{s}\right)}{u_{m \nu}\left(z_{s}\right)} \frac{\sqrt{k_{m \nu}}}{\sqrt{k_{n, \nu-1}}} & m \neq n\end{cases}
$$


Here, the terms $\kappa_{m n}^{R_{\nu}}$ and $\kappa_{m n}^{T_{\nu}}$ are given by Equations (3.62) and (3.64), respectively. Similar to the plane wave case, these matrices can be incorporated into the recursive formulae given by Equations (3.65) and (3.66) to compute the reflection and transmission coefficients at an interface corresponding to any region in the discretized medium given knowledge of the transmission coefficients for the region containing the acoustic source.

The reformulation of the transmission coefficients via the matrix in Equation (3.72) allows for the definition of a forward transmission loss equation for the discretized range-dependent cylindrical waveguide with azimuthal symmetry. This transmission loss equation is given by the expression:

$$
T L(r, z)=-20 \log _{10}\left|\sqrt{\frac{2 \pi}{r}} \sum_{m=1}^{N} B_{m \nu} u_{m \nu}(z) \frac{u_{m \nu}\left(z_{s}\right)}{\rho\left(z_{s}\right) \sqrt{k_{m \nu}}} e^{i k_{m \nu} r}\right| ; \quad r \in\left[r_{\nu}, r_{\nu+1}\right),
$$

where the terms $B_{m \nu}$ are computed recursively by generalizing the results in Equations (3.66) and (3.72) to $N$ modes. Equation (3.74) accounts for losses due to cylindrical spreading in the waveguide as well as non-adiabatic mode coupling via the transmission coefficients $B_{m \nu}$. While this equation is correct relative to the physical assumptions made, we will see in the next section that adjustments are necessary to ensure numerical stability of the solution for all ranges and depths.

\subsection{Reformulation for Numerical Stability}

When implementing the transmission loss solution given by Equation (3.74) via Equations (3.66) and (3.72) into a discrete computing environment there are two terms that require special attention to maintain numerical stability. The first is the exponential term that appears in the entries of the transmission coefficient matrix in Equation (3.72). The numerical instability in this term becomes obvious if one rewrites the term as:

$$
e^{i\left(k_{n, \nu-1}-k_{m \nu}\right) r_{\nu}}=e^{i k_{n, \nu-1} r_{\nu}}\left[e^{-i k_{m \nu} r_{\nu}}\right]
$$


When the mode of index $m$ in the above expression transitions from propagating to evanescent, its associated eigenvalue, $k_{m \nu}$, becomes purely imaginary. Under these conditions, the exponential on the left side of this expression becomes numerically unstable at large values of $r_{\nu}$ due to the fact that the term in brackets on the right grows without bound. The instability in this term can be removed if we distribute the exponential term from the forward transmission loss equation in Equation (3.74) inside the expression for the transmission coefficient $B_{m \nu}$ given by Equations (3.66) and (3.72). Doing so, we see that the exponential term in the entries of the transmission coefficient matrix become

$$
e^{i k_{n, \nu-1} r_{\nu}}\left[e^{-i k_{m \nu} r_{\nu}}\right] \times e^{i k_{m \nu} r}=e^{i k_{n, \nu-1} r_{\nu}}\left[e^{i k_{m \nu}\left(r-r_{\nu}\right)}\right] .
$$

Since $r_{\nu}$ corresponds to the range at the beginning of the horizontal region $\nu$ and $r$ corresponds to ranges within said region, we have that $\left(r-r_{\nu}\right) \geq 0$. As such, the exponential term in Equation (3.76) is numerically stable since the term in brackets on the right side of this equation decays exponentially with increasing range from the interface located at $r=r_{\nu}$.

The second numerically unstable term with which we must concern ourselves is the eigenfunction ratio that appears in the entries of the matrix in Equation (3.72). This term is written

$$
\frac{u_{n, \nu-1}\left(z_{s}\right)}{u_{m \nu}\left(z_{s}\right)} \frac{\sqrt{k_{m \nu}}}{\sqrt{k_{n, \nu-1}}} .
$$

It is clear from Equation (3.77) that for certain source depths (i.e. those corresponding to the zeros of the eigenfunction $u_{m \nu}$ ) the above term will become undefined due to division by zero. To remove this instability we again turn our attention to the forward transmission loss equation in Equation (3.74). If we distribute the term $\left(u_{m \nu}\left(z_{s}\right) / \rho\left(z_{s}\right) \sqrt{k_{m \nu}}\right)$ from this equation inside the expression for the transmission coefficient $B_{m \nu}$ given by Equations (3.66) and (3.72), the term in 
Equation (3.77) becomes:

$$
\frac{u_{n, \nu-1}\left(z_{s}\right)}{u_{m \nu}\left(z_{s}\right)} \frac{\sqrt{k_{m \nu}}}{\sqrt{k_{n, \nu-1}}} \times \frac{u_{m \nu}\left(z_{s}\right)}{\rho\left(z_{s}\right) \sqrt{k_{m \nu}}}=\frac{u_{n, \nu-1}\left(z_{s}\right)}{\rho\left(z_{s}\right) \sqrt{k_{n, \nu-1}}} .
$$

It is apparent from Equation (3.78) that this term is now stable for all source depths.

Taking these results into account, we now wish to define a new set of expressions for the numerically stable reformulation of the presented asymptotic stepwise coupled mode solution. We will begin by defining the following expression corresponding to the initial transmission coefficients existing in the horizontal partition containing the acoustic source:

$$
B_{m 0}^{S}=B_{m 0} e^{i k_{m 0} r_{0}} \frac{u_{m 0}\left(z_{s}\right)}{\rho\left(z_{s}\right) \sqrt{k_{m 0}}}
$$

where $B_{m 0}$ are the initial transmission coefficients appearing in Equation (3.66). Given this definition, the recursive matrix solution for the numerically stable transmission coefficients is given by the expression

$$
\left(\begin{array}{c}
B_{1 \nu}^{S} \\
B_{2 \nu}^{S}
\end{array}\right)=T_{\nu}^{S}\left(\begin{array}{c}
B_{1, \nu-1}^{S} \\
B_{2, \nu-1}^{S}
\end{array}\right)=\prod_{\sigma=1}^{\nu} T_{\sigma}^{S}\left(\begin{array}{c}
B_{10}^{S} \\
B_{20}^{S}
\end{array}\right)
$$

where

$$
T_{\nu}^{S}=\left(\begin{array}{cc}
\kappa_{11}^{T_{\nu}} e^{i k_{1 \nu}\left(r-r_{\nu}\right)}\left\langle u_{1, \nu-1}, u_{1 \nu}\right\rangle & \kappa_{12}^{T_{\nu}} e^{i k_{1 \nu}\left(r-r_{\nu}\right)}\left\langle u_{2, \nu-1}, u_{1 \nu}\right\rangle \\
\kappa_{21}^{T_{\nu}} e^{i k_{2 \nu}\left(r-r_{\nu}\right)}\left\langle u_{1, \nu-1}, u_{2 \nu}\right\rangle & \kappa_{22}^{T_{\nu}} e^{i k_{2 \nu}\left(r-r_{\nu}\right)}\left\langle u_{2, \nu-1}, u_{2 \nu}\right\rangle
\end{array}\right)
$$

Finally, we may define the following numerically stable equivalent of the forward transmission loss equation in Equation (3.74):

$$
T L^{S}(r, z)=-20 \log _{10}\left|\sqrt{\frac{2 \pi}{r}} \sum_{m=1}^{N} B_{m \nu}^{S} u_{m \nu}(z)\right| ; \quad r \in\left[r_{\nu}, r_{\nu+1}\right)
$$


where the terms $B_{m \nu}^{S}$ in the summation in Equation (3.82) are obtained by generalizing the results of Equations (3.79)-(3.81) to $N$ modes. Going forward we wish to verify this result for several range-dependent bottom depth problems of interest.

\subsection{Mode Turnaround and Eigenvalue Lag Distance}

An additional numerical issue that requires special treatment when implementing the presented solution is that of mode turnaround. Mode turnaround occurs when a mode's grazing angle begins to approach $90^{\circ}$ in the vicinity of its cutoff depth. Due to the stepwise nature of the approach presented here, this phenomenon creates a discontinuity in the transmission coefficients computed via the matrix in Equation (3.72). As discussed in Section (2.3), when a mode becomes fully cutoff its eigenvalue becomes purely imaginary and thus its real part is zero. To understand how this affects the transmission coefficient calculation we turn our attention to the terms $\kappa_{n n}^{T_{\nu}}$, which appear as multipliers in the diagonal terms of the matrix in Equation (3.72). Recalling Equation (3.64), these terms are given by the expression:

$$
\kappa_{n n}^{T_{\nu}}=\frac{2 k_{n, \nu-1}}{\left(k_{n, \nu-1}+k_{n \nu}\right)} .
$$

If we consider the case of upslope propagation, we see that the term $k_{n \nu}$ in this expression will become imaginary in the vicinity of mode n's cutoff depth prior to the term $k_{n, \nu-1}$, as the latter term lags the former by a distance of $\Delta r_{\nu-1}$. This lag distance between the eigenvalues of the adjacent regions $\nu-1$ and $\nu$ will create a temporary peak in the term $\kappa_{n n}^{T_{\nu}}$ in the vicinity of the range at which mode $n$ turns around. This peak will, in turn, create a discontinuity in the transmission coefficient associated with mode $n$, as evident from the matrix in Equation (3.72). Figure (3.4) provides a depiction of the lag distance between the eigenvalues $k_{n, \nu-1}$ and $k_{n \nu}$ in the vicinity of a mode turning around in an upslope propagation scenario. As can be seen in this figure, the real part of the eigenvalue $k_{n \nu}$ becomes 
zero a short distance before that of the eigenvalue $k_{n, \nu-1}$. In Figure (3.5a) a plot is provided of a mode's grazing angle approaching $90^{\circ}$ as it becomes cutoff in an upsloping waveguide. The discontinuity created in the transmission coefficient associated with this mode is then depicted in Figure (3.5b). The result of the

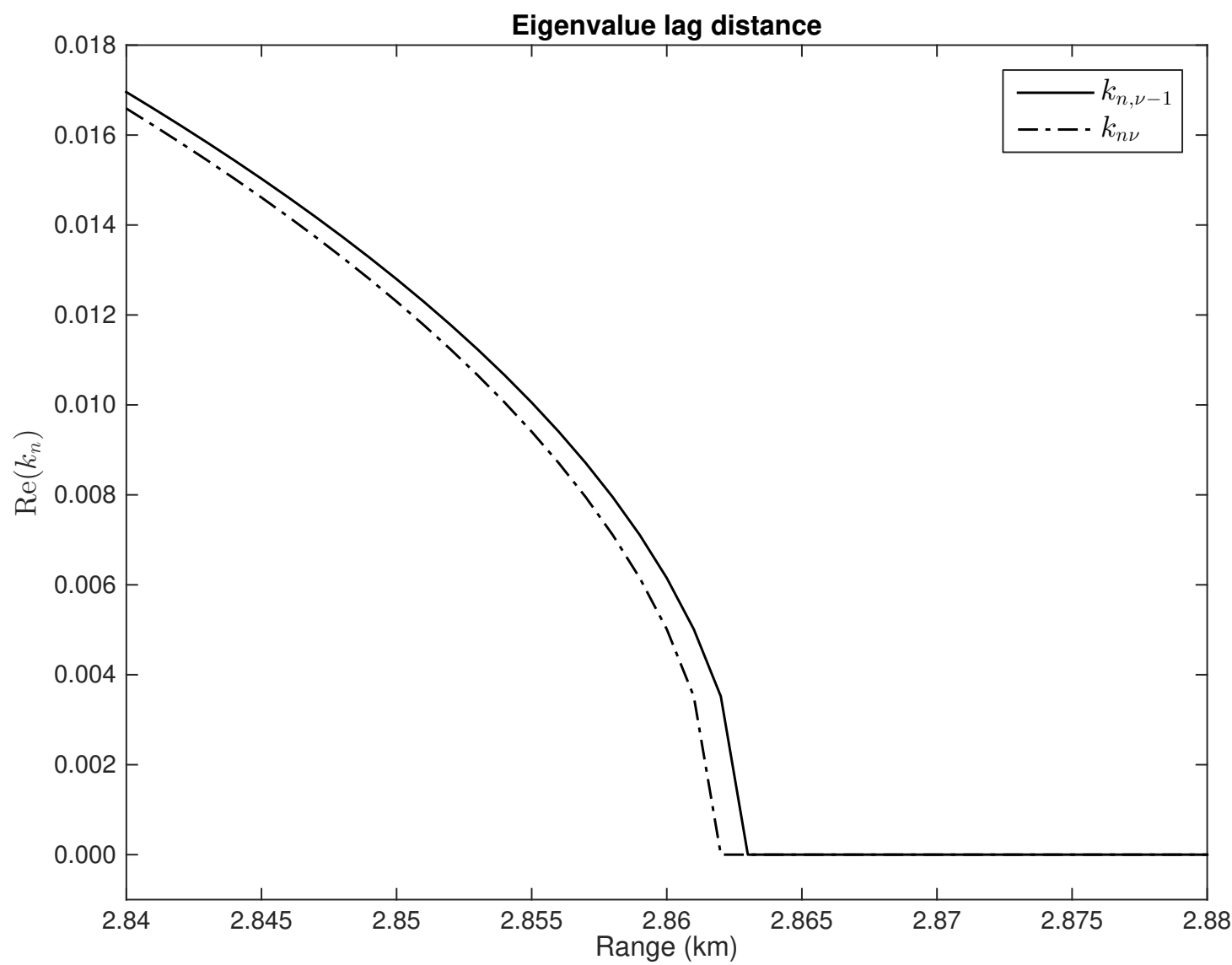

Figure 3.4: Depiction of eigenvalue lag distance in the vicinity of a mode's turnaround point in an upslope propagation scenario. As can be seen, the real part of the eigenvalue $k_{n \nu}$ becomes zero before that of the eigenvalue $k_{n, \nu-1}$ due to the small distnace, $\Delta r_{\nu-1}$, separating the respective regions in which the two eigenvalues exist.

discontinuity in the transmission coefficients as depicted in Figure (3.5b) is that artificial peaks will appear in the transmission loss curves computed via Equation (3.82) in the vicinity of mode cutoff depths. There are a number of viable approaches for removing these artificial peaks. A simple approach is to maintain a 


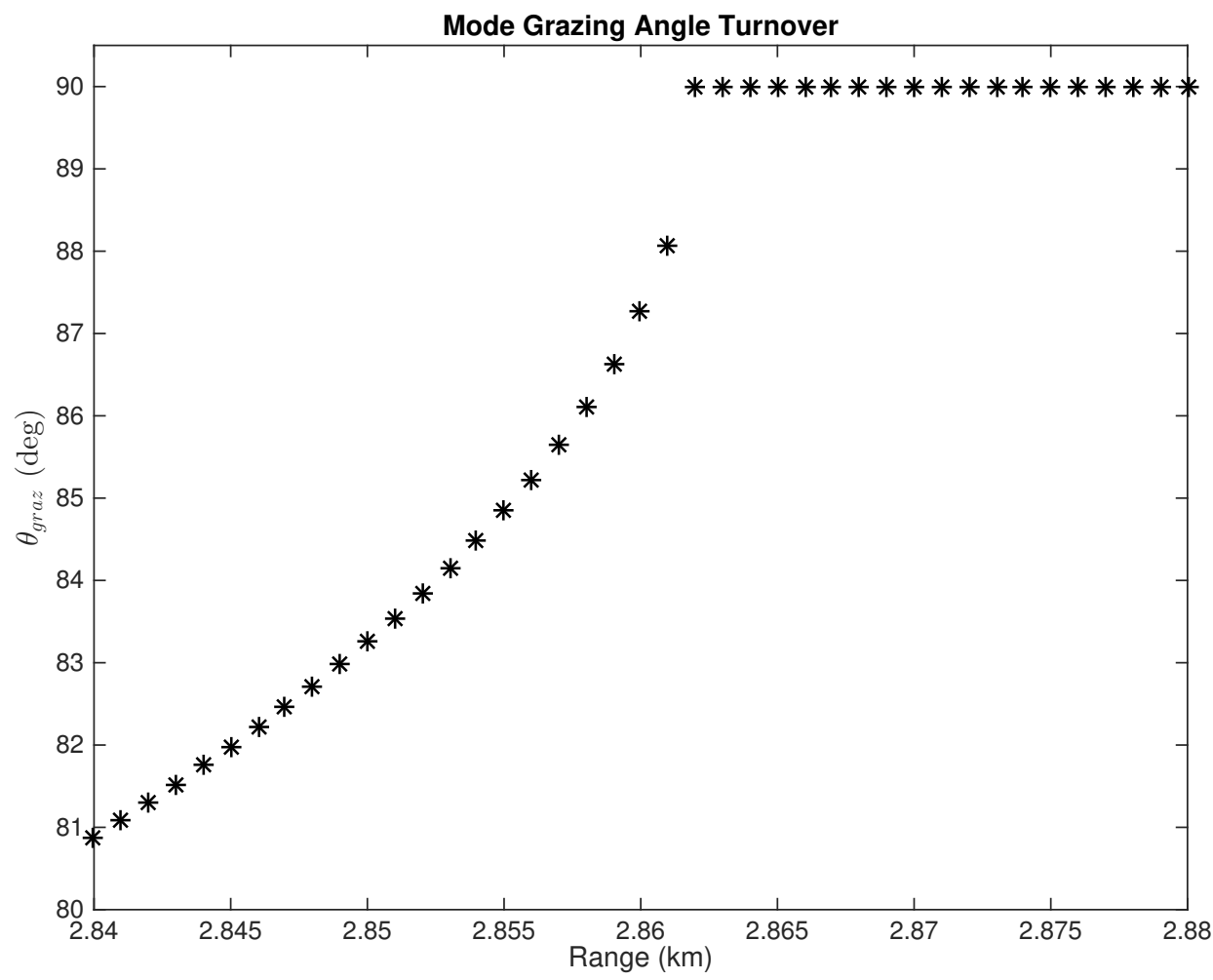

(a)

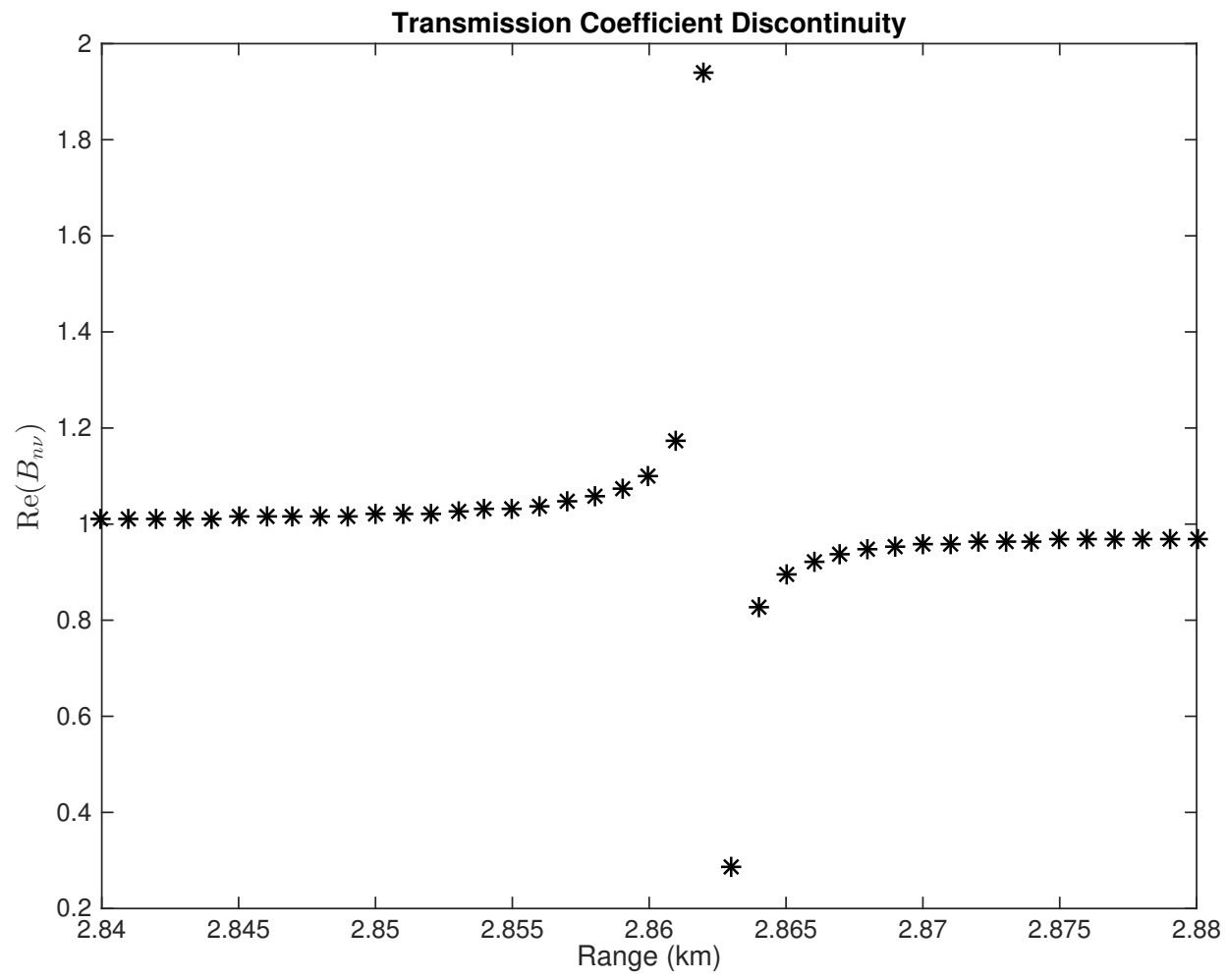

(b)

Figure 3.5: (a) Depiction of a mode's grazing angle approaching $90^{\circ}$ near its cutoff in an upslope propagation scenario (b) Discontinuity in the transmission coefficient associated with a cutoff mode 
running average of the term $\kappa_{n n}^{T_{\nu}}$ for each mode. These average values of $\kappa_{n n}^{T_{\nu}}$ may then be used as surrogate multipliers in the diagonal terms of the matrix given by Equation (3.72) for range intervals containing mode cutoff depths. In Figure (3.6a) a transmission loss curve produced using Equation (3.82) is provided for an upslope propagation scenario. Distinct peaks can be seen in this figure as higher order modes are cutoff due to the rapid decrease in waveguide depth. Figure (3.6b) shows a corrected version of the transmission loss curve for this propagation scenario where the peaks have been removed using the previously suggested moving average method. 


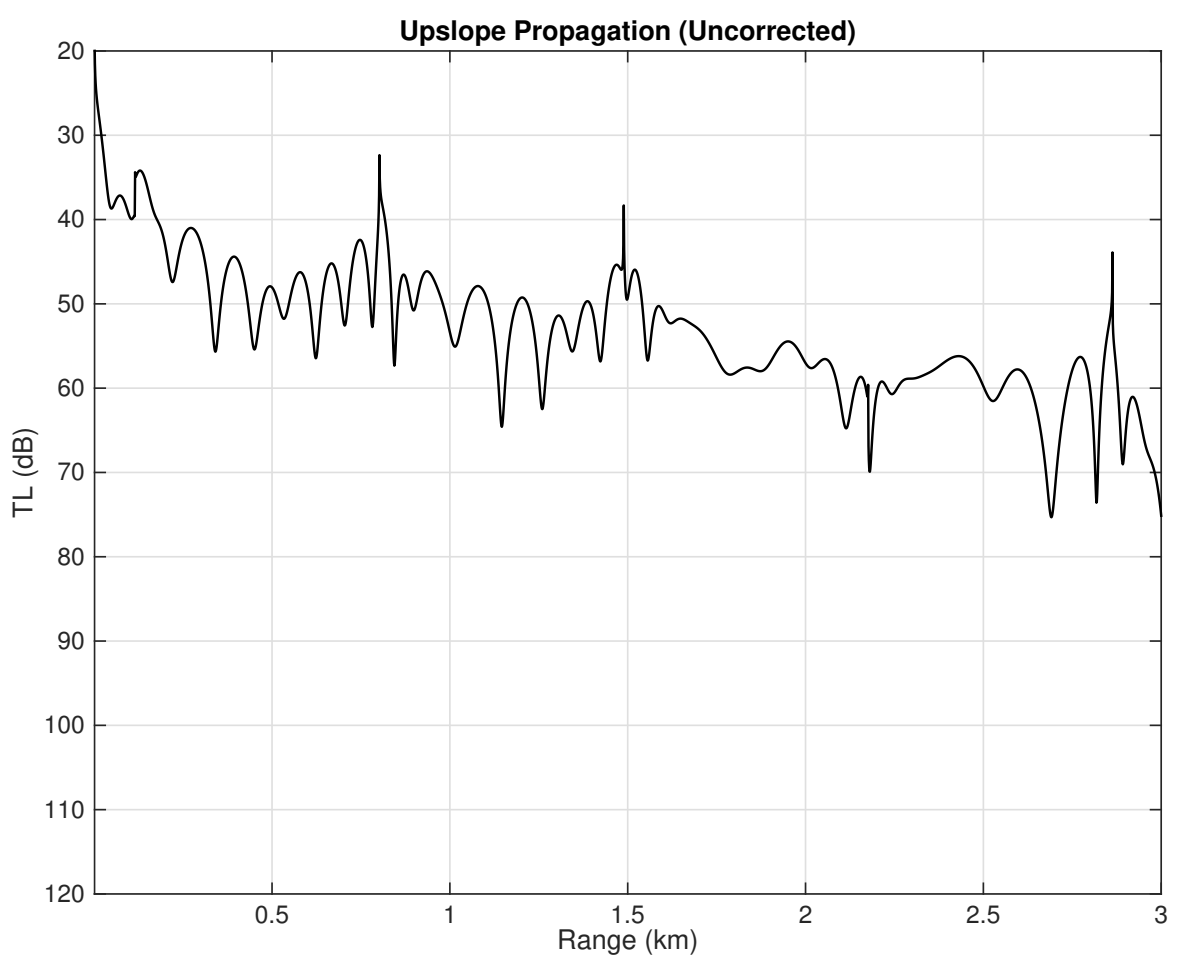

(a)

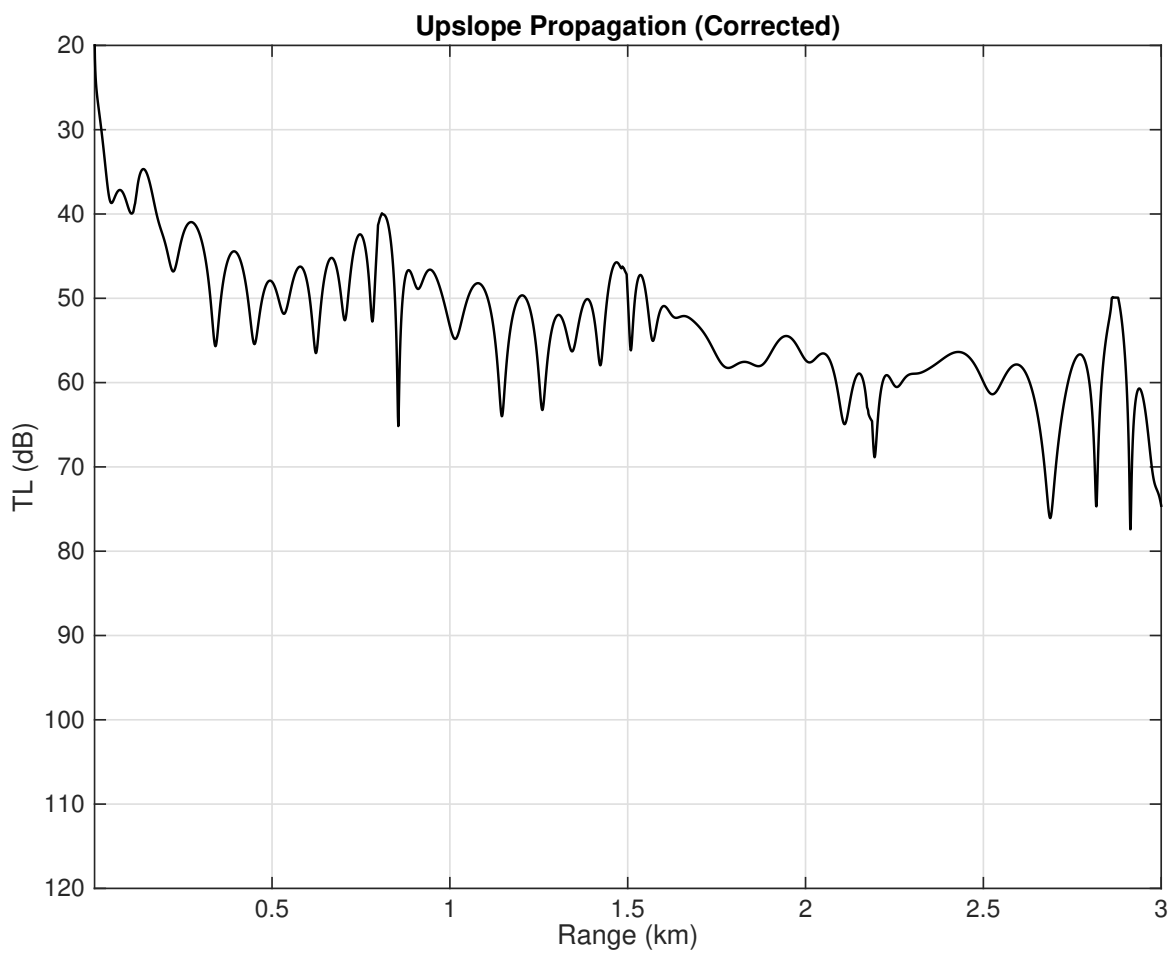

(b)

Figure 3.6: (a) Depiction of artificial peaks appearing in the transmission loss estimate for an upslope propagation scenario due to mode cutoff (b) Removal of artificial peaks in the transmission loss curve via a moving average applied to the term $\kappa_{n n}^{T_{\nu}}$ of the matrix in Equation (3.72) in the vicinity of mode cutoff depths 


\section{List of References}

[1] G. A. Leibiger, "Wave propagation in an inhomogeneous medium with slow spatial variation," Ph.D. dissertation, Stevens Institute of Technology, Castle Point, Hoboken, New Jersey, 1968. 


\section{CHAPTER 4 \\ Verification of Theory}

\subsection{Background}

To verify the efficacy of the presented theory a particular problem of interest was chosen: modeling mode coupling due to horizontally-variable bottom depth in a rigid-bottom waveguide. As discussed in Section (3.3), an environment with general range-dependent bottom depth may be approximated by a series of connected wedge subenvironments. Moreover, each wedge subenvironment is purely specified by an initial bottom depth and a bottom slope angle. Taking this into account, a set of test cases was constructed that spans all possible combinations of two-slope propagation environments. It is proposed that the bottom depth for any environment may be approximated by a combination of these two-slope scenarios. To support this claim, one test case was constructed that consists of a bottom depth profile approximated by seven slopes.

For all scenarios a sound speed in water of $1500 \mathrm{~m} \mathrm{~s}^{-1}$ and a water column density of $1000 \mathrm{~kg} \mathrm{~m}^{-3}$ were chosen. To run these scenarios, a normal modes propagation model was developed in MATLAB [1] that implements the discretization technique described in Section (3.3). Output from this model is a set of transmission loss curves produced using the forward transmission loss equation given in Equation (3.82). Non-adiabatic mode coupling is accounted for in the model through the recursive computation of transmission coefficients at discrete interfaces throughout the medium using Equations (3.80). Going forward, this model will be referred to as the Asymptotic Stepwise Coupled Mode (ASCM) model. 


\subsection{Comparison to Benchmark Solution}

To verify the transmission loss estimates generated by the ASCM model, the same propagation scenarios were modeled using COUPLE, a benchmark solution for range-dependent problems developed by Evans [2], which was first discussed in Section (2.7). To ensure that the rigid-bottom boundary condition was being correctly represented in COUPLE, a test was performed whereby a rigid flatbottom propagation scenario described by Jensen, Kuperman, Porter \& Schmidt [3] was modeled repeatedly while increasing the sound speed and density in a horizontal layer representing the rigid basement. The sound speed and density for this basement layer were considered sufficient once no detectable difference existed between the transmission loss curve generated by COUPLE and that given in reference [3]. This resulted in the basement layer having a sound speed of $13000 \mathrm{~m} \mathrm{~s}^{-1}$ and a density of $3500 \mathrm{~kg} \mathrm{~m}^{-3}$.

In what follows, two figures are provided for each propagation scenario modeled using the ASCM and COUPLE solutions. The first figure provides a plot of the bottom depth over range for each scenario. In this figure, the source and receiver depths are also depicted using a circle and star, respectively. The second figure provides a comparison of the transmission loss curves produced by the ASCM and COUPLE models for each scenario. Above each of the figures additional information about the propagation scenario is provided. This information

includes the source frequency, $f$, the bottom slope angle, $\beta$, the fixed point (source) depth, Fxd, and the moving point (receiver) depth, Mvd. 


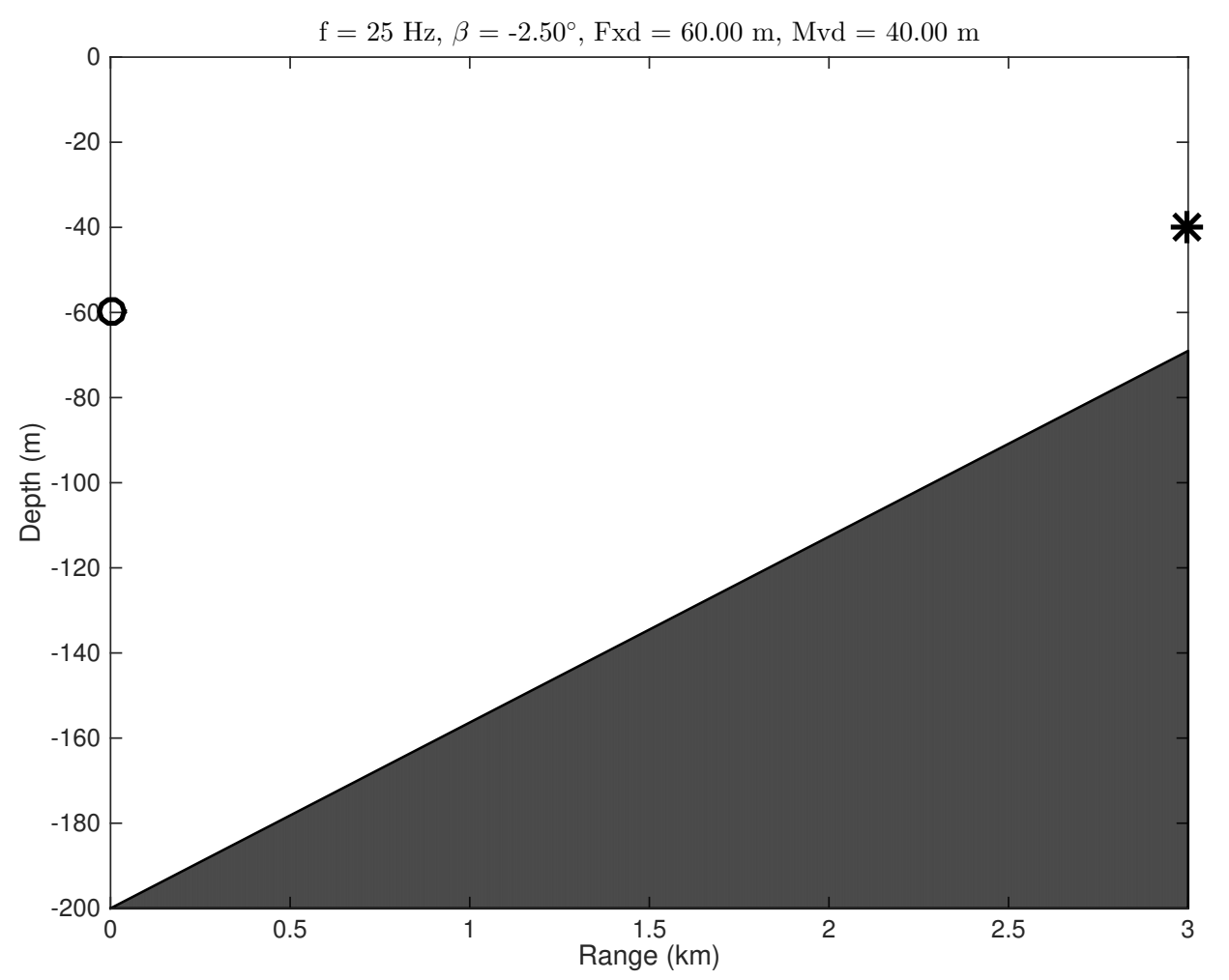

(a)

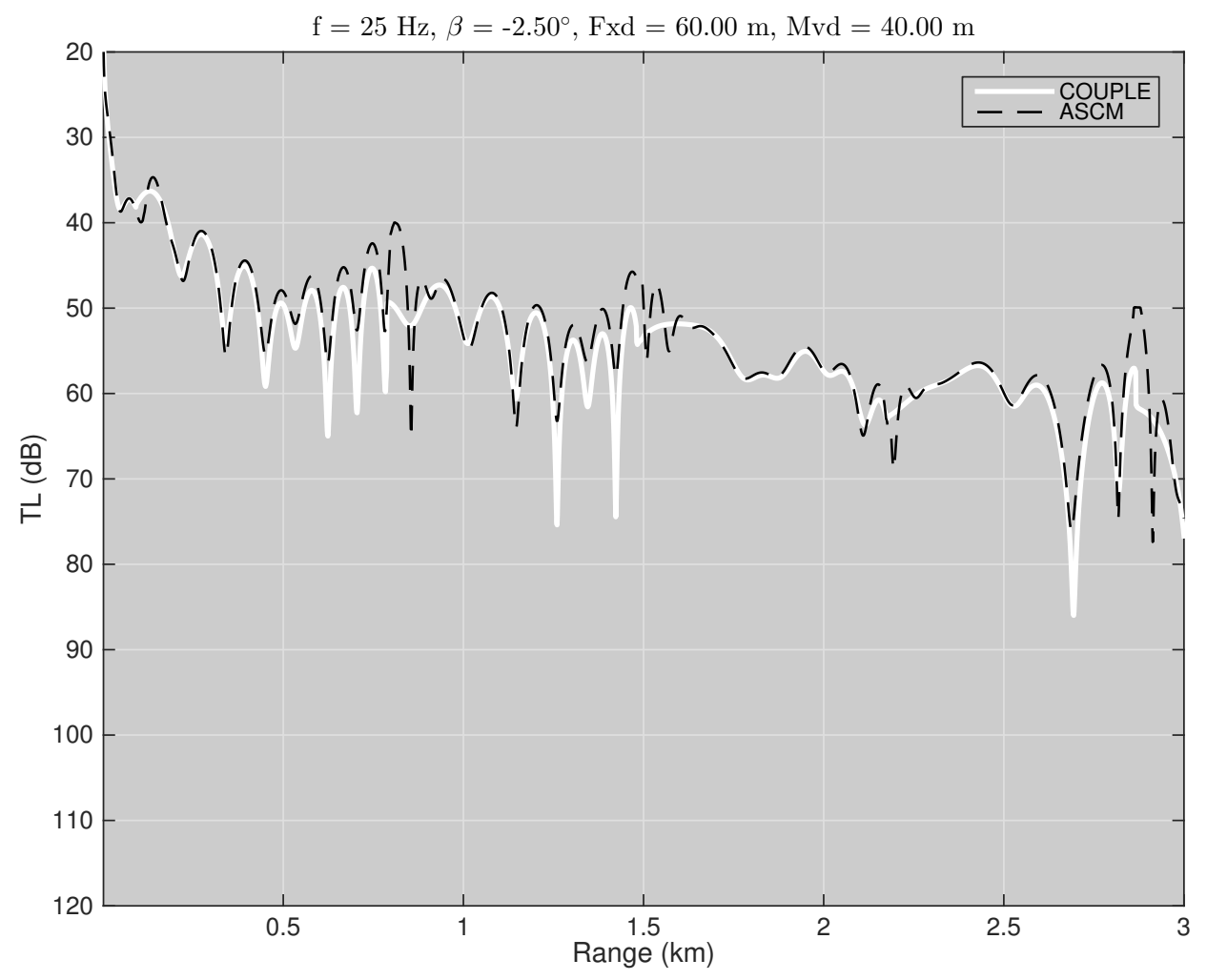

(b)

Figure 4.1: (a) Upslope propagation scenario (b) Comparison of transmission loss estimates produced by the ASCM and COUPLE propagation models for the scenario depicted in (a) 


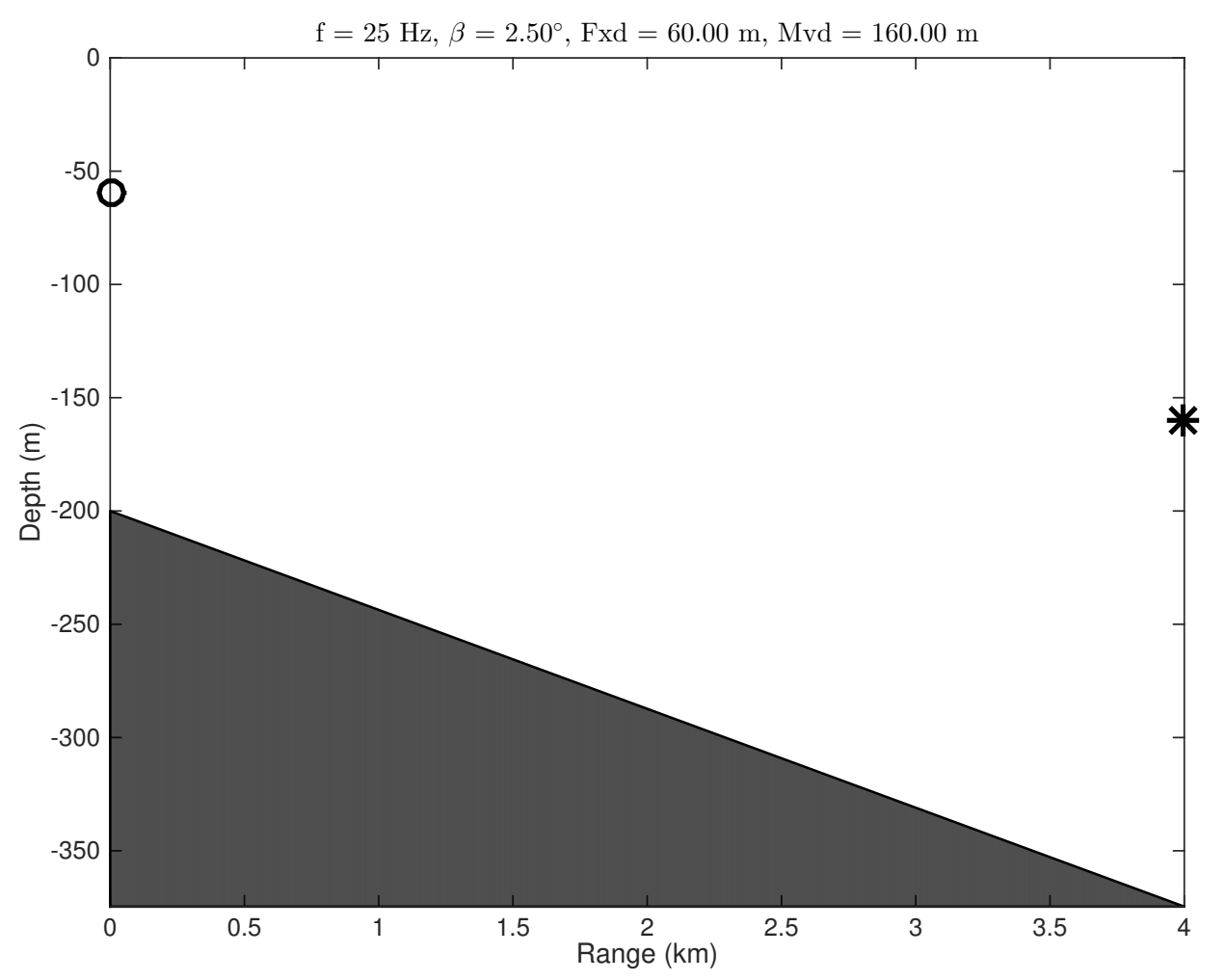

(a)

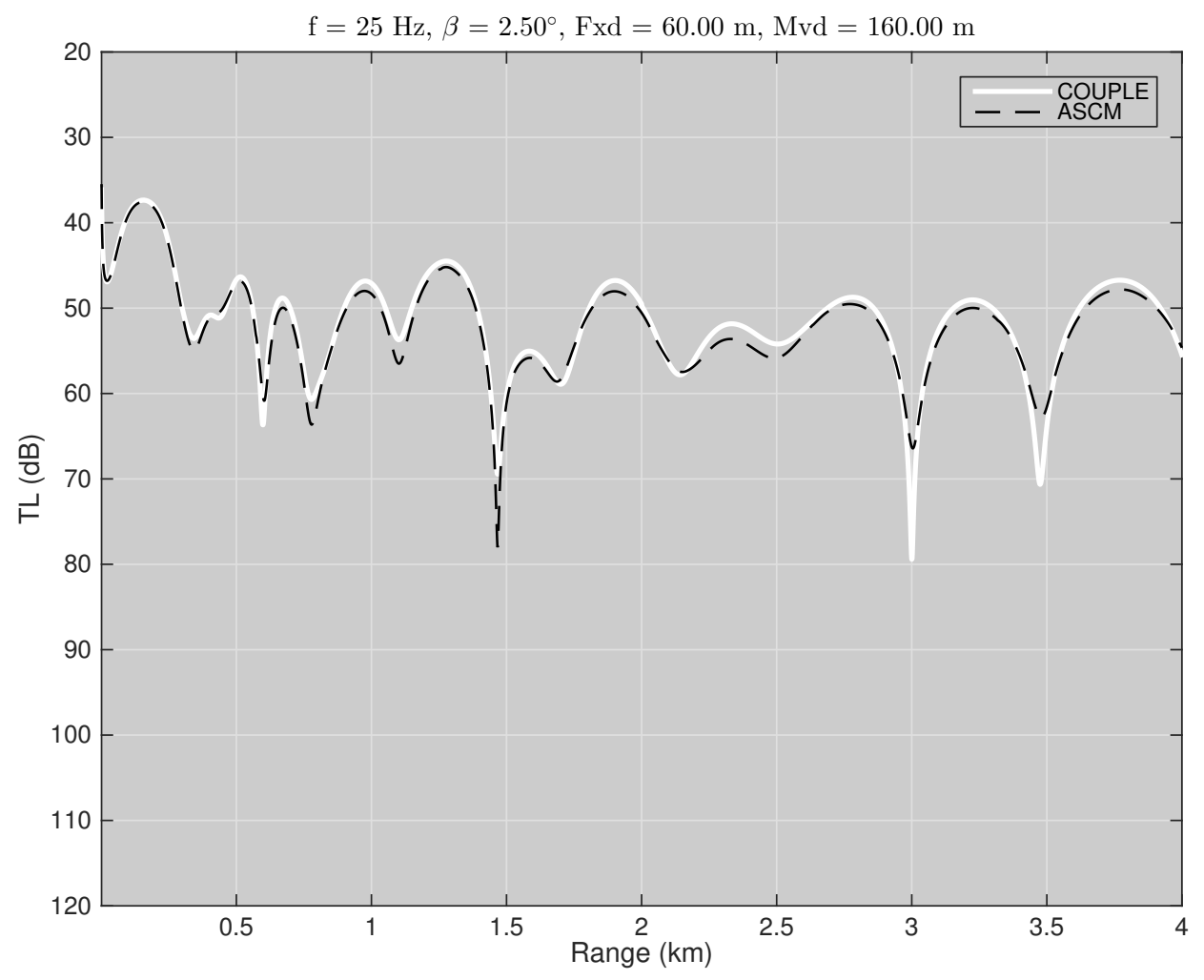

(b)

Figure 4.2: (a) Downslope propagation scenario (b) Comparison of transmission loss estimates produced by the ASCM and COUPLE propagation models for the scenario depicted in (a) 


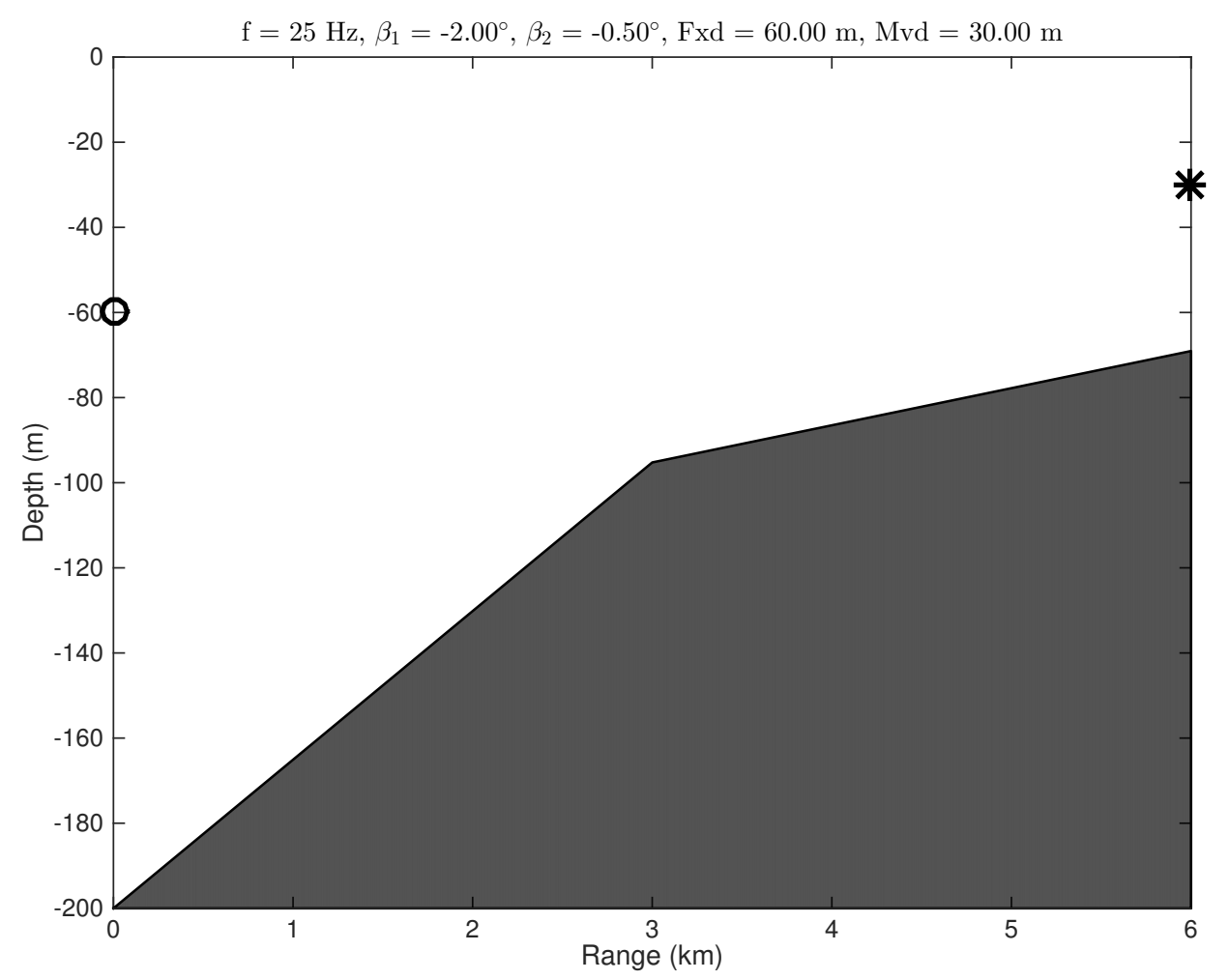

(a)

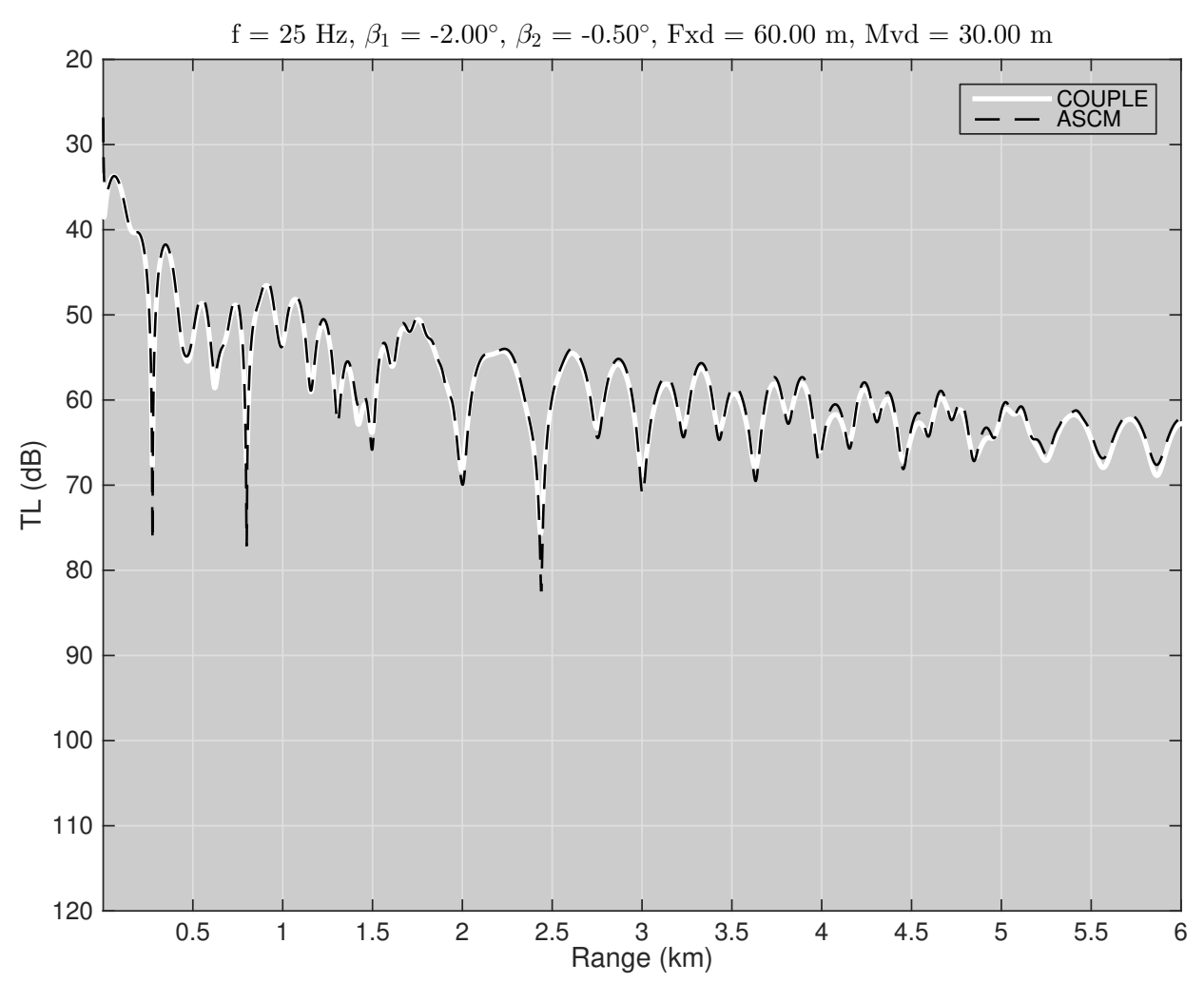

(b)

Figure 4.3: (a) Two-slope uphill propagation scenario (b) Comparison of transmission loss estimates produced by the ASCM and COUPLE propagation models for the scenario depicted in (a) 


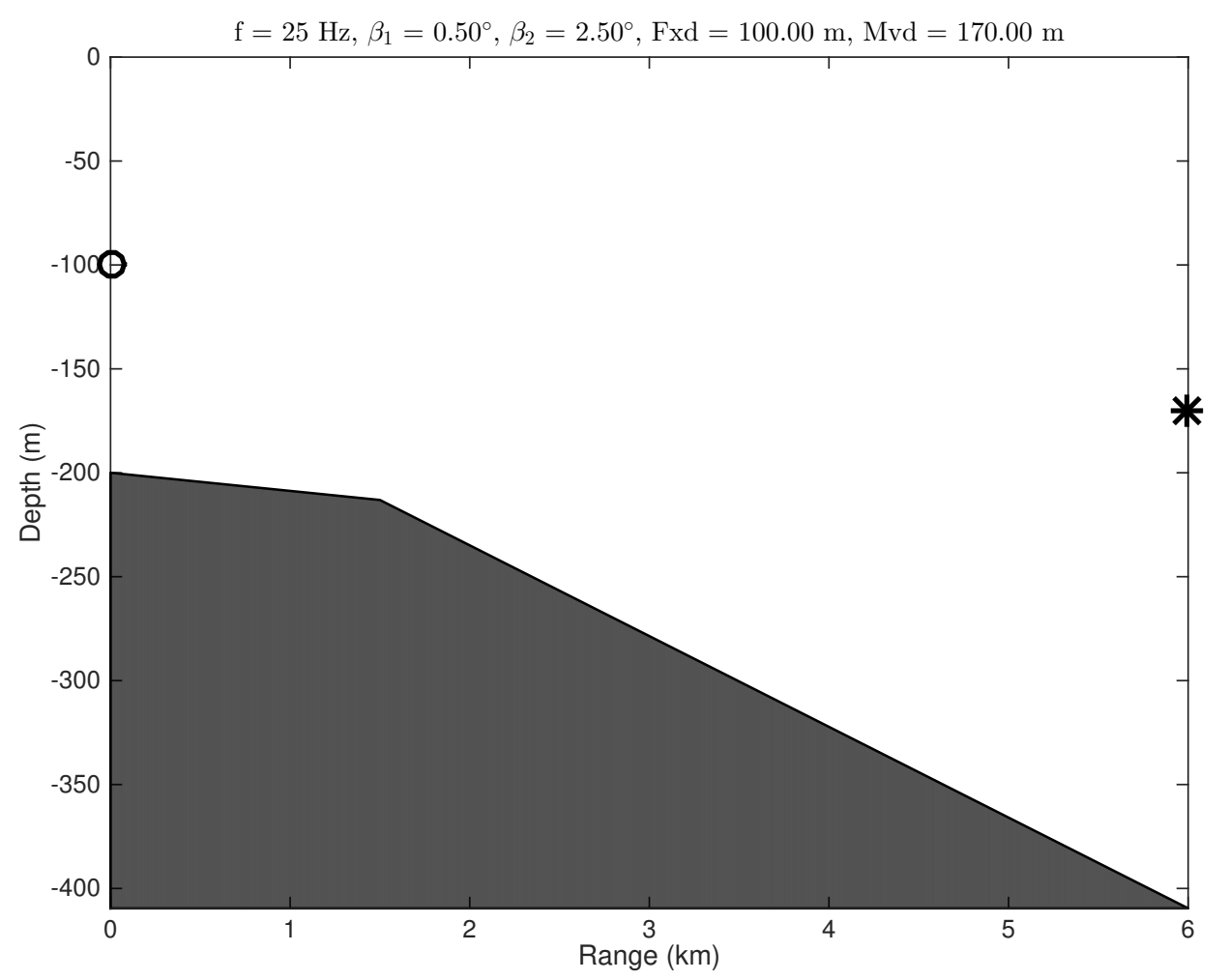

(a)

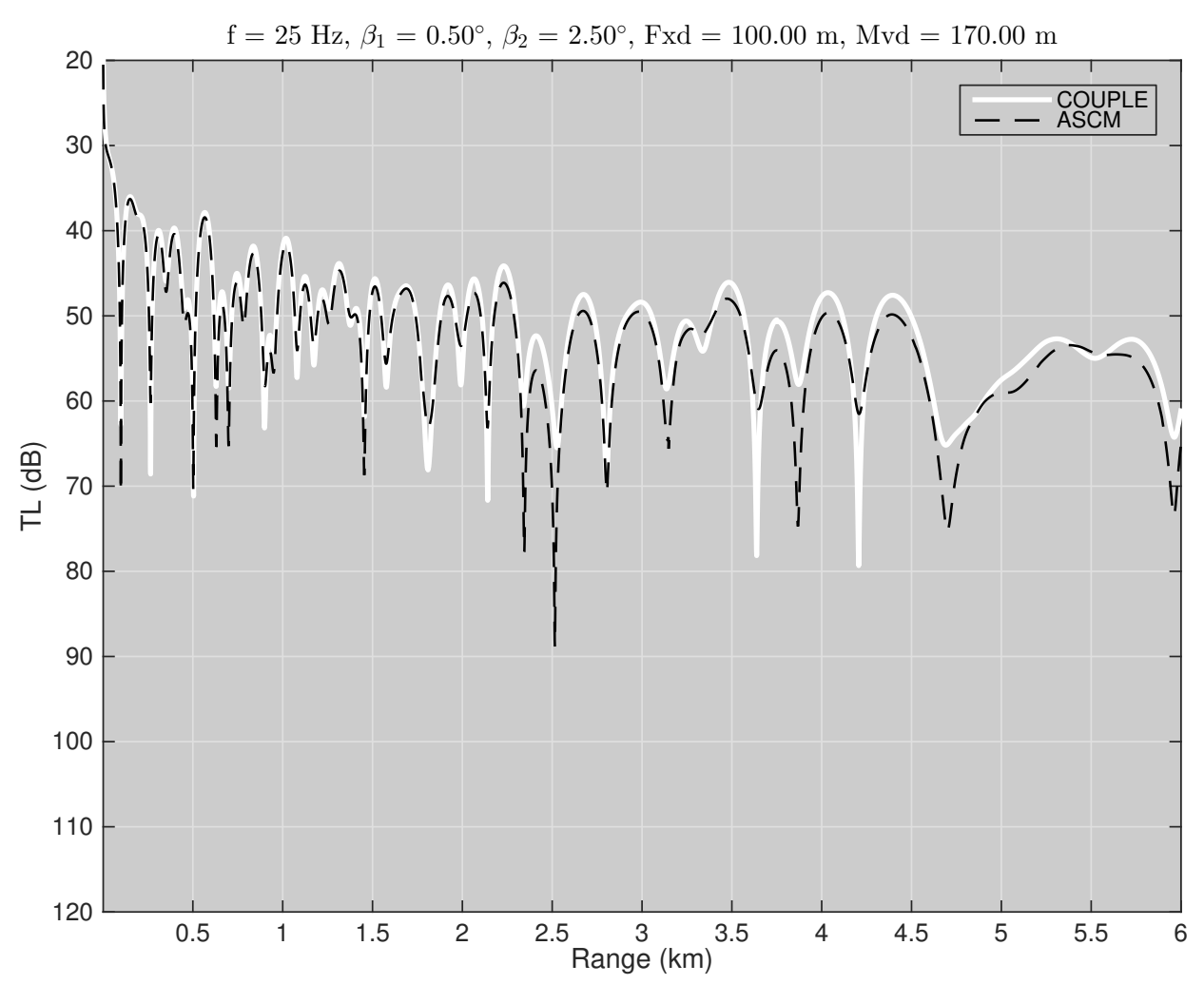

(b)

Figure 4.4: (a) Two-slope downhill propagation scenario (b) Comparison of transmission loss estimates produced by the ASCM and COUPLE propagation models for the scenario depicted in (a) 


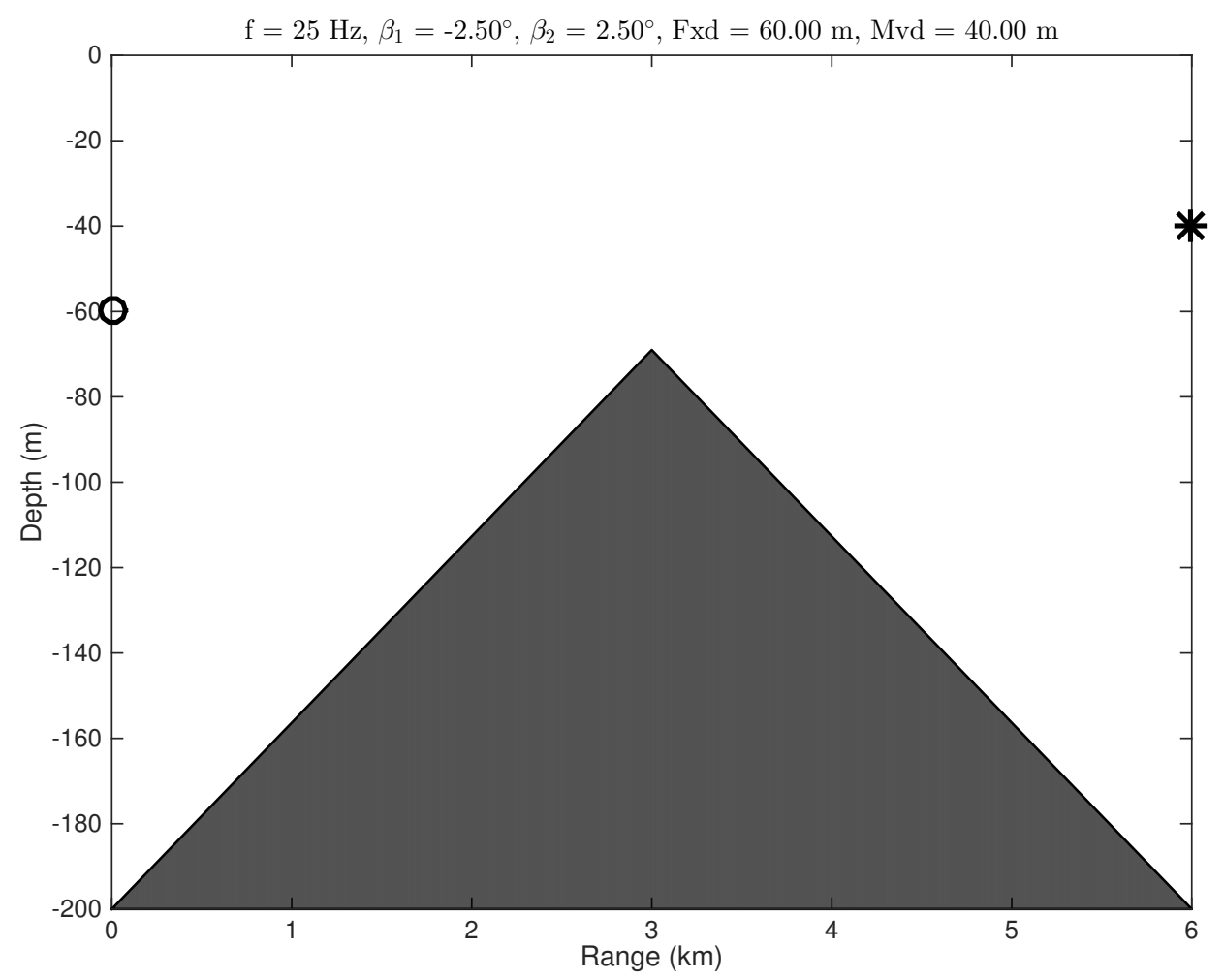

(a)

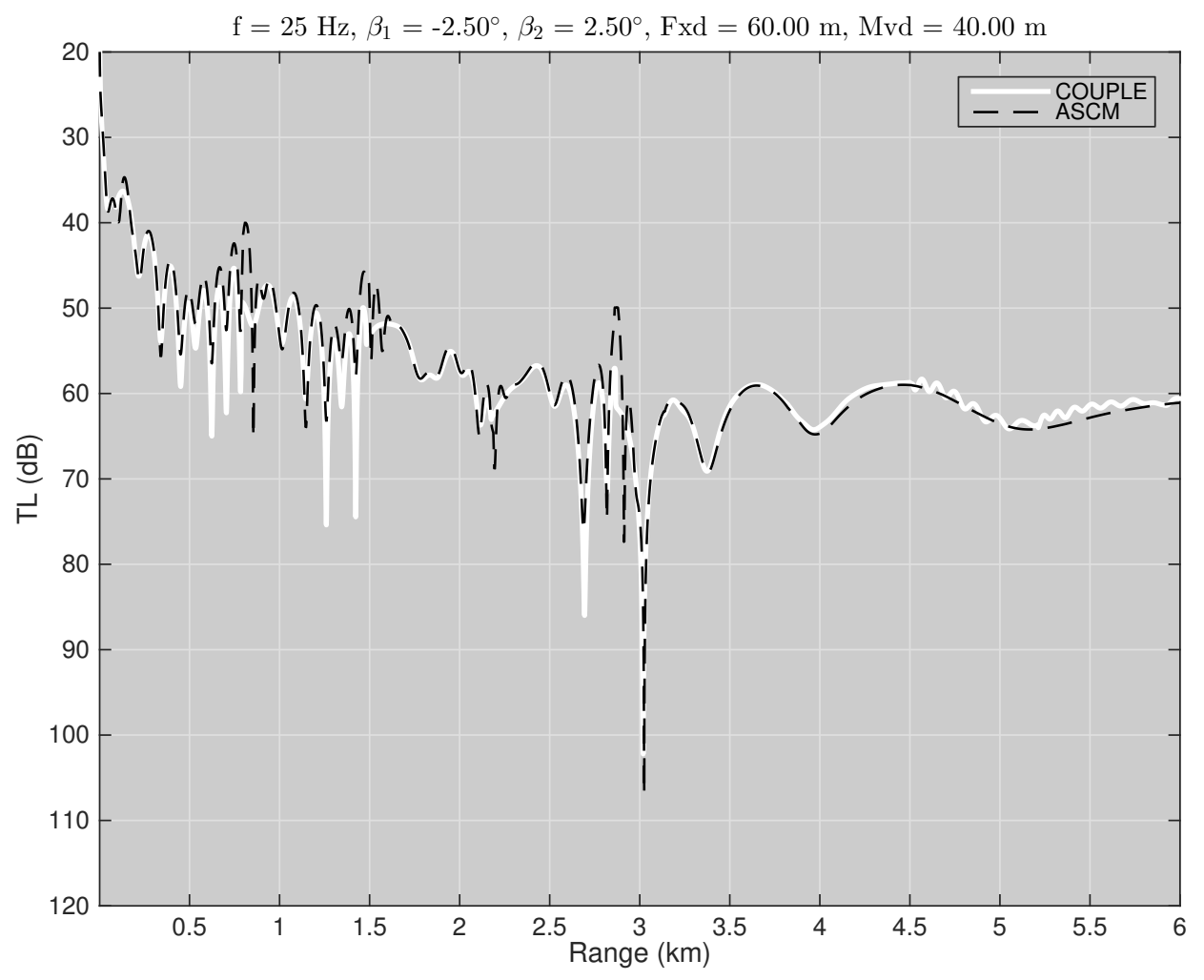

(b)

Figure 4.5: (a) 2D seamount propagation scenario (b) Comparison of transmission loss estimates produced by the ASCM and COUPLE propagation models for the scenario depicted in (a) 


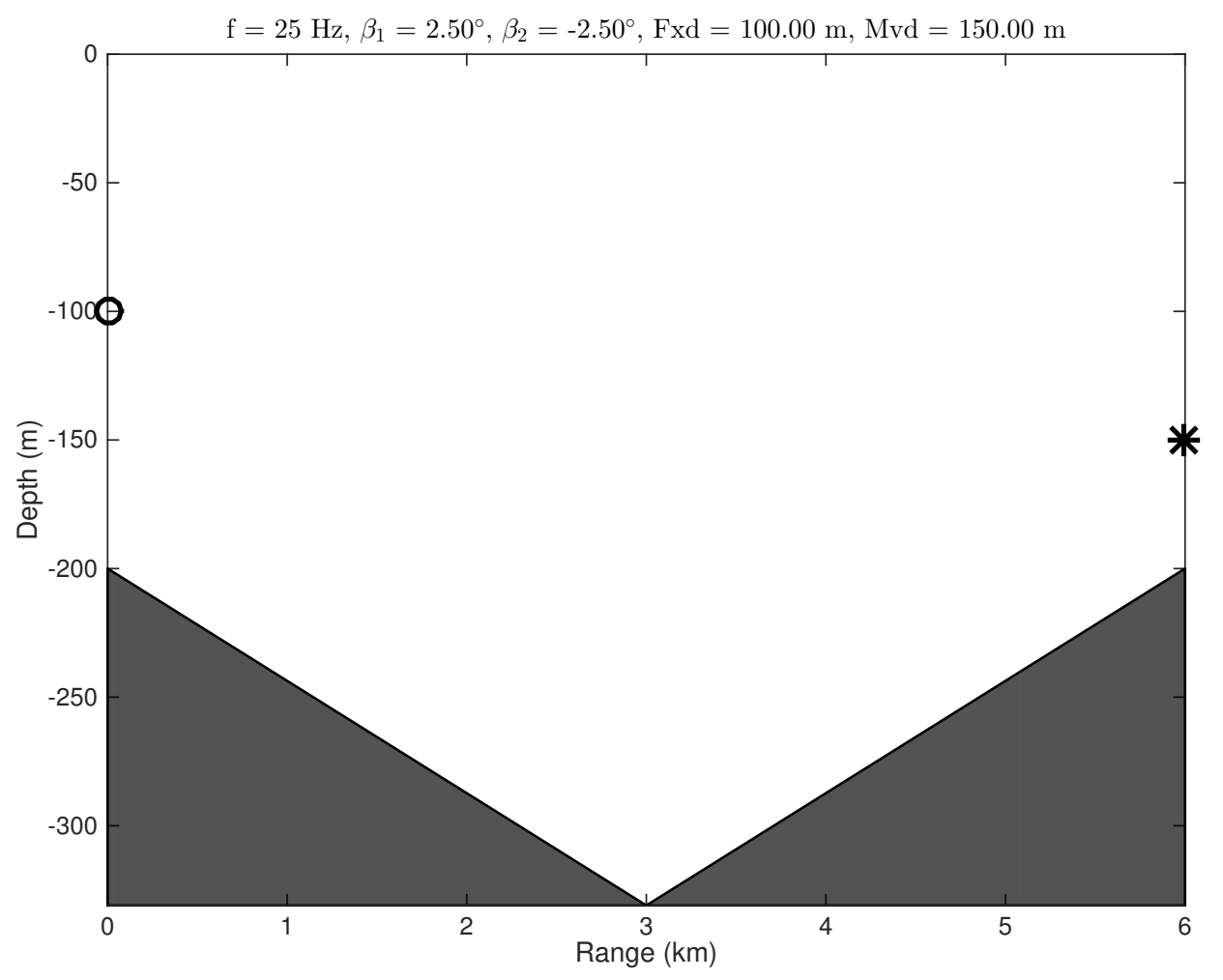

(a)

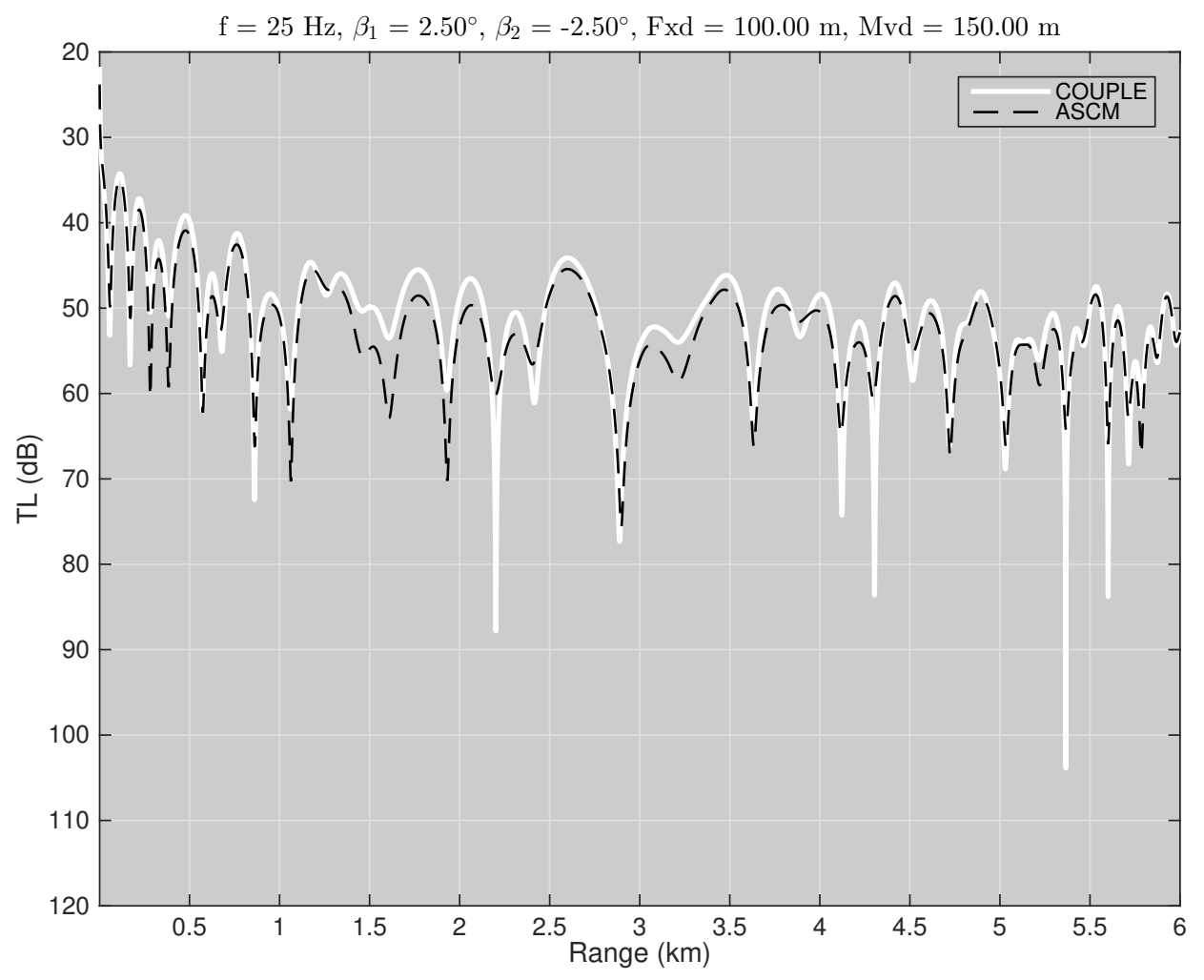

(b)

Figure 4.6: (a) 2D canyon propagation scenario (b) Comparison of transmission loss estimates produced by the ASCM and COUPLE propagation models for the scenario depicted in (a) 


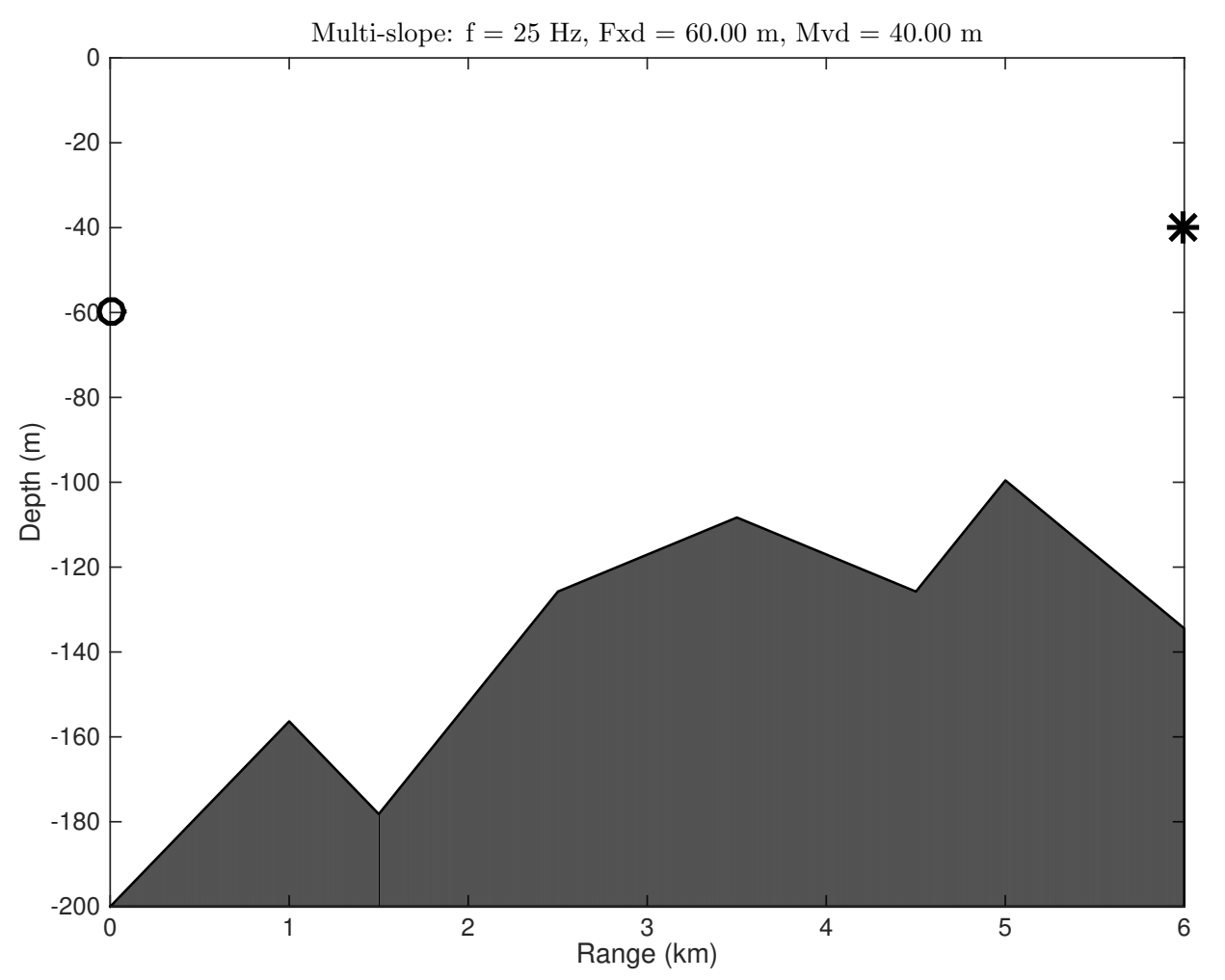

(a)

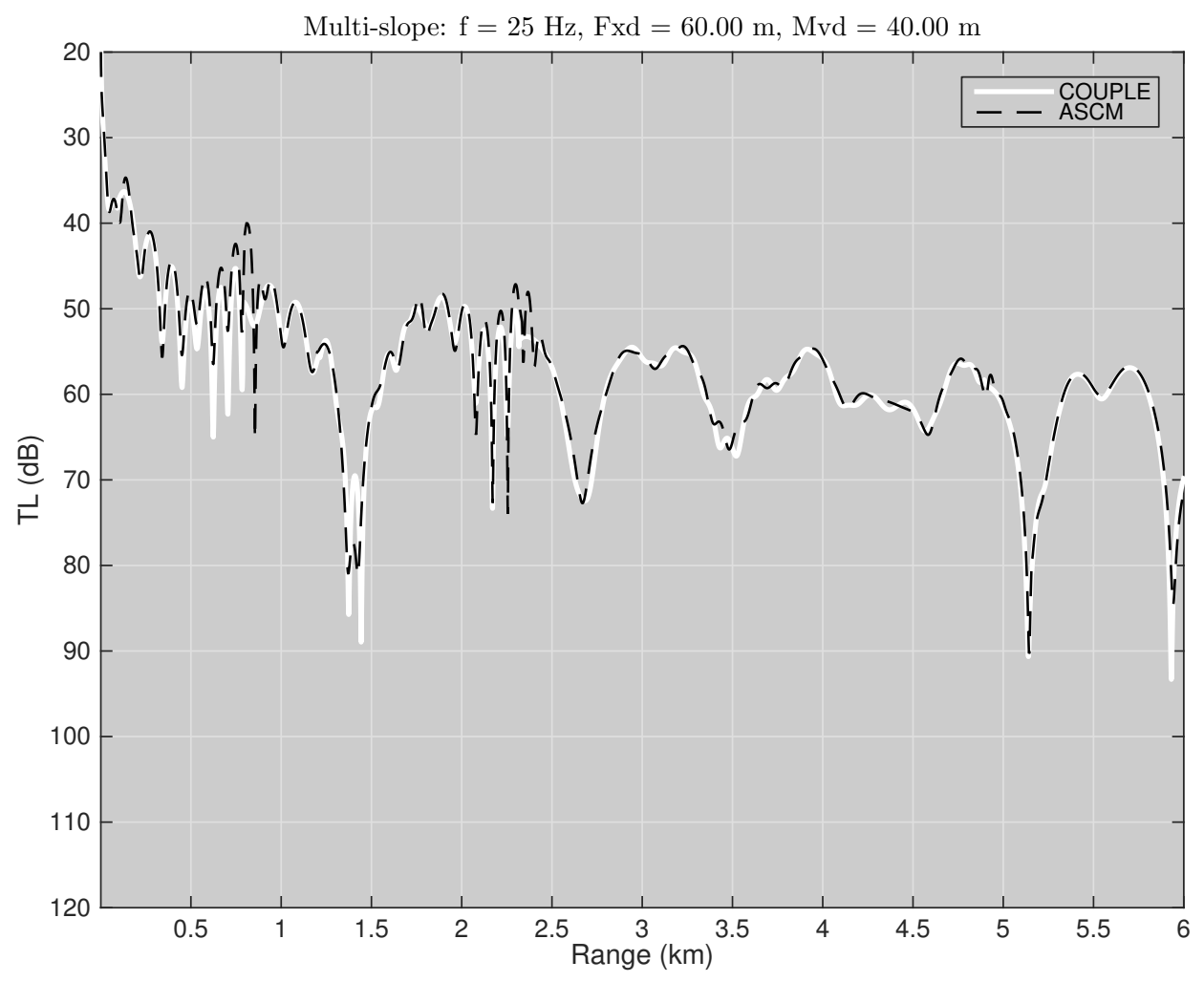

(b)

Figure 4.7: (a) General multi-slope propagation scenario (b) Comparison of transmission loss estimates produced by the ASCM and COUPLE propagation models for the scenario depicted in (a) 


\subsection{Comparison to Parabolic Equation Solution}

While the strong peak-to-peak agreement achieved with the benchmark solution is sufficient for verifying the feasibility of the proposed coupled-mode approach, comparisons with solutions not based on normal mode theory were also pursued. One such comparison was made with the Monterey-Miami Parabolic Equation (MMPE) model developed by Smith [4]. The MMPE solution uses a split-step Fourier algorithm to solve the parabolic wave equation. This algorithm involves decomposing the acoustic field into a slowly modulating envelope function, known as the PE field function, and a phase term, which oscillates at the acoustic frequency of the source.

The use of recurrent Fast Fourier Transforms in the MMPE solution necessitates approximating the transition over the water-sediment interface with a smooth function. As such, the MMPE model did not allow for the representation of a perfectly-rigid bottom boundary condition as used in the test cases described in Section (4.2). To get around this limitation the source frequency was increased from $25 \mathrm{~Hz}$ to $200 \mathrm{~Hz}$, which greatly reduced, but did not eliminate, the loss off acoustic energy to the sub-basement. Despite this difference in bottom boundary conditions, appreciable agreement was obtained between the benchmark (COUPLE), ASCM, and MMPE solutions for this scenario. Figures (4.8a)-(4.8d) provide a three-way comparison of the transmission loss estimates produced by the three solutions for the 2D seamount test case with a $200 \mathrm{~Hz}$ source. Figure (4.9) provides a plot of the MMPE-predicted complex pressure field, which shows energy losses in the MMPE pseudo-rigid basement. 


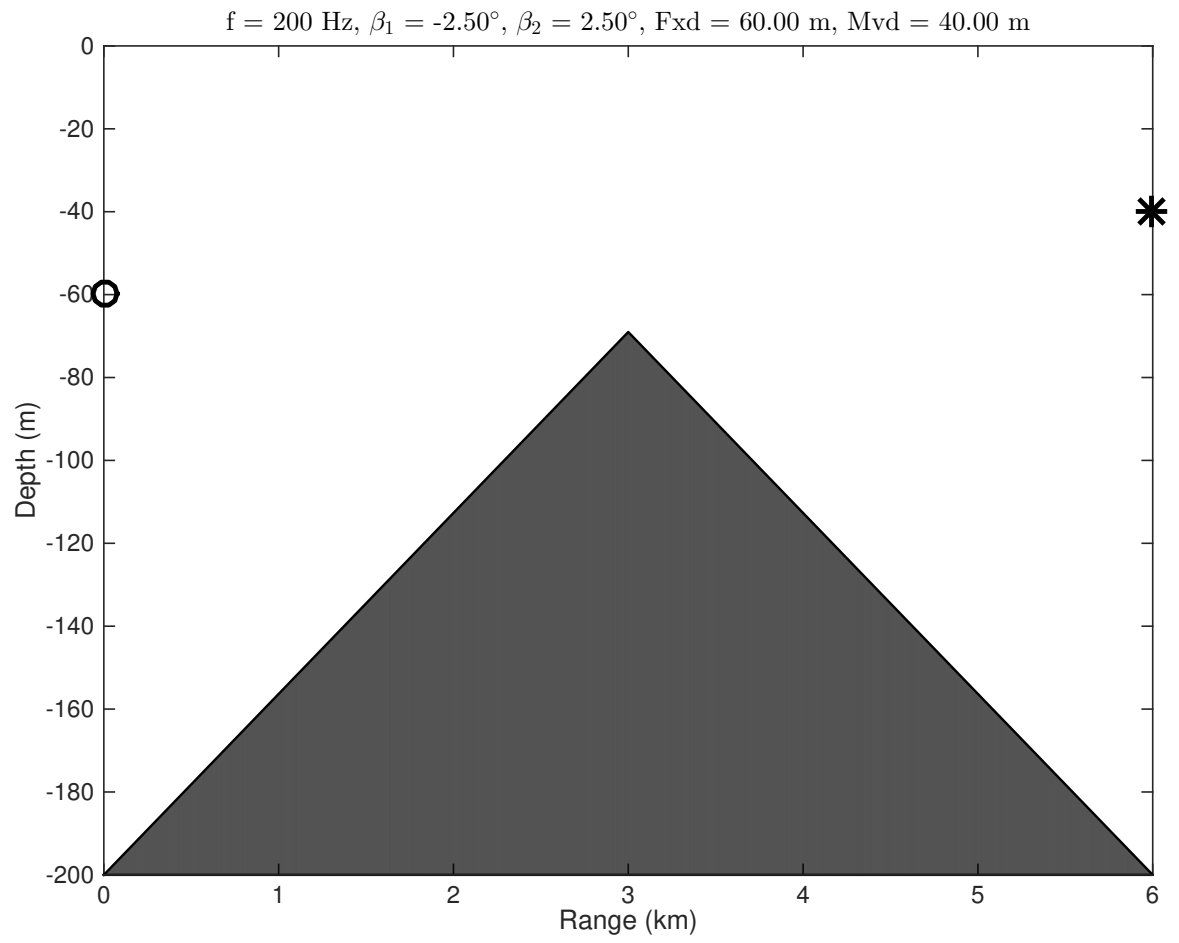

(a) High Frequency 2D Seamount Propagation Scenario

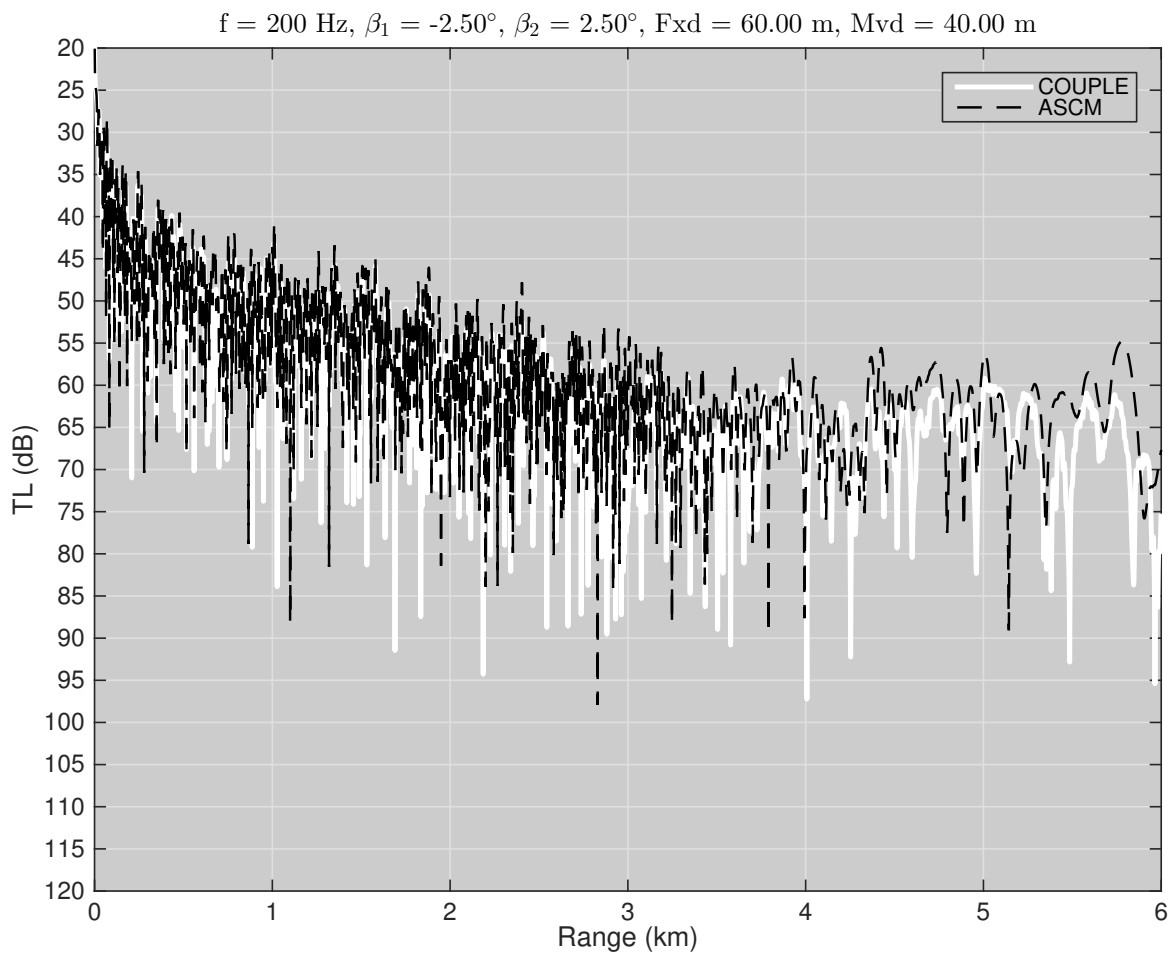

(b) ASCM vs. COUPLE Transmission Loss Comparison 


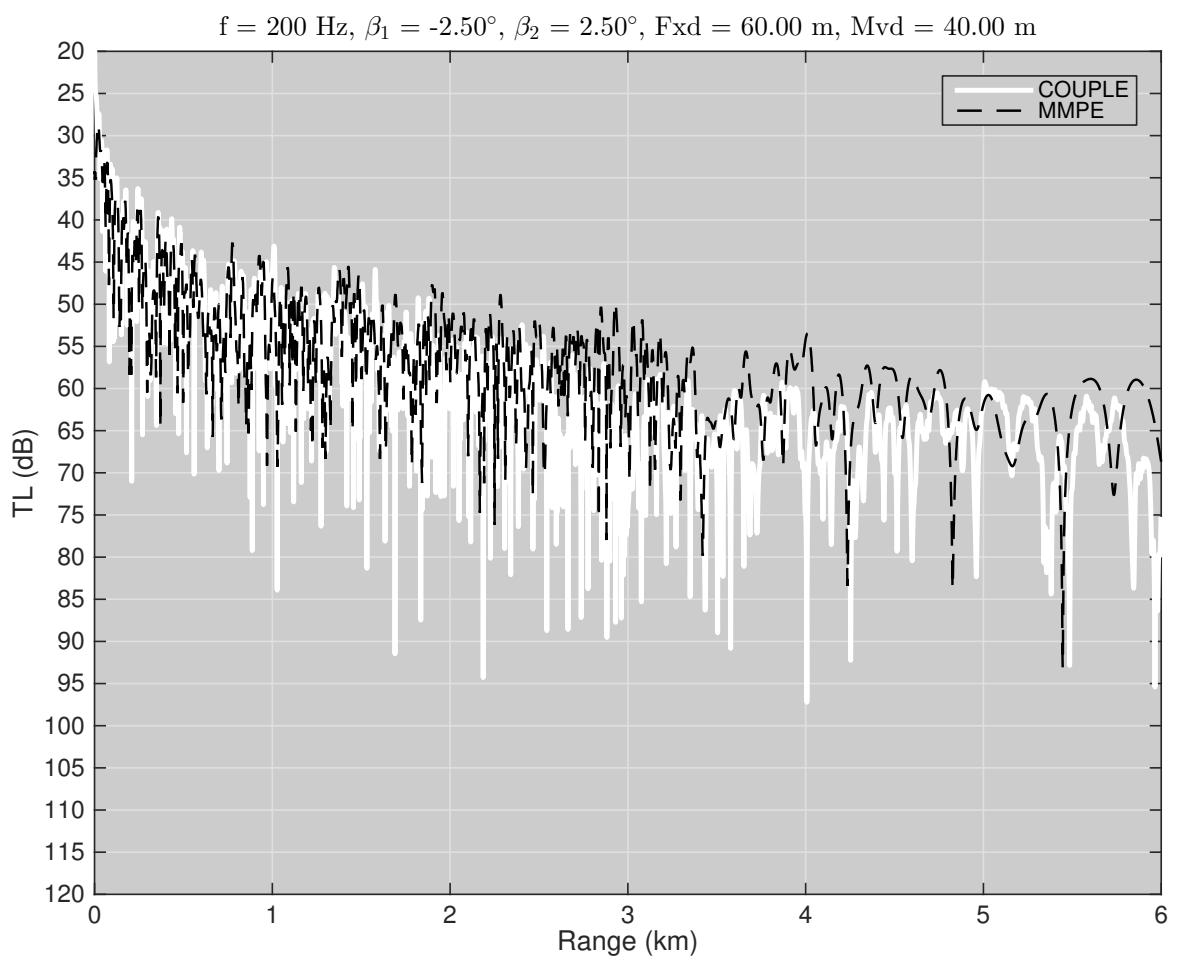

(c) MMPE vs. COUPLE Transmission Loss Comparison

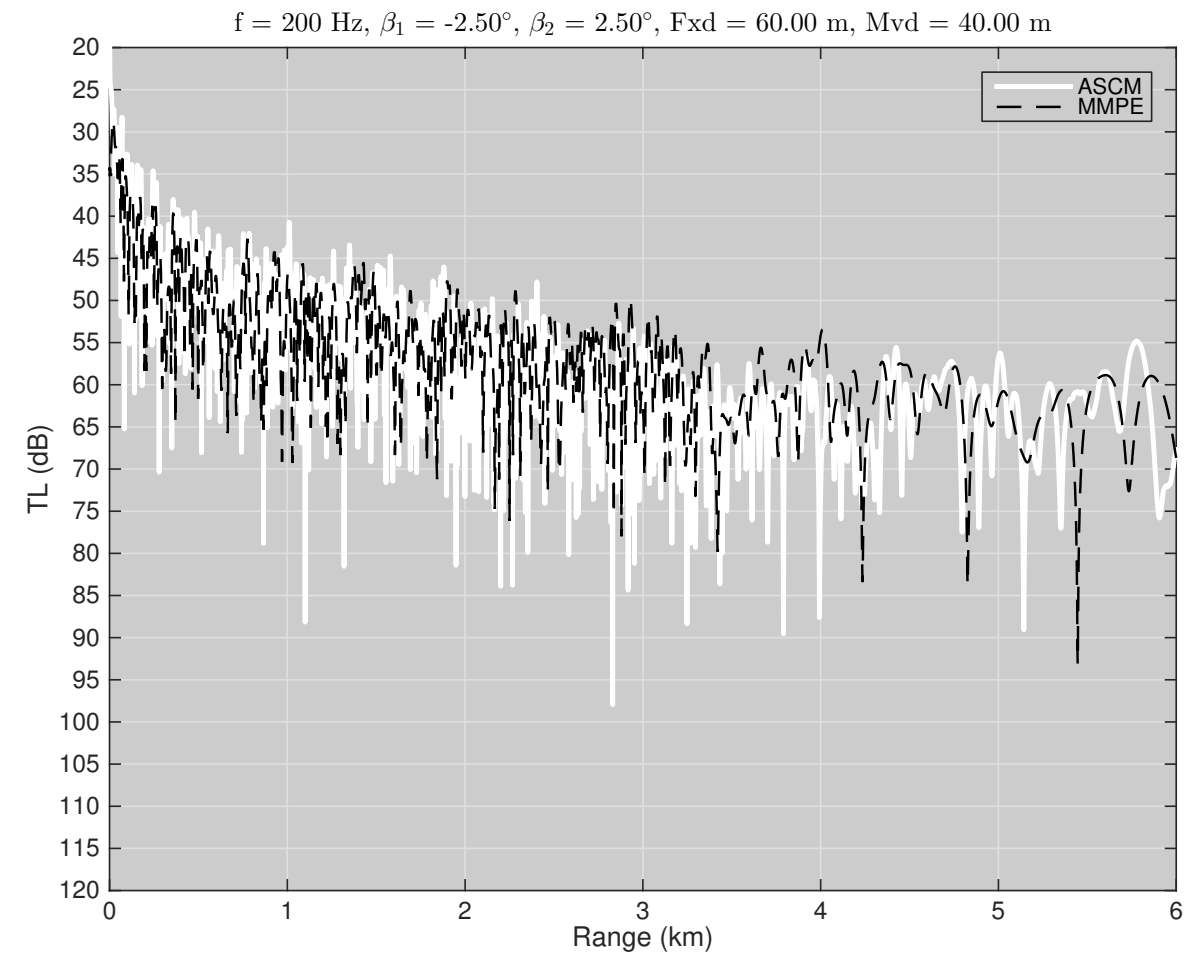

(d) MMPE vs. ASCM Transmission Loss Comparison

Figure 4.8: Comparison of transmission loss estimates between COUPLE, ASCM, and MMPE propagation models for the high frequency 2D seamount scenario 


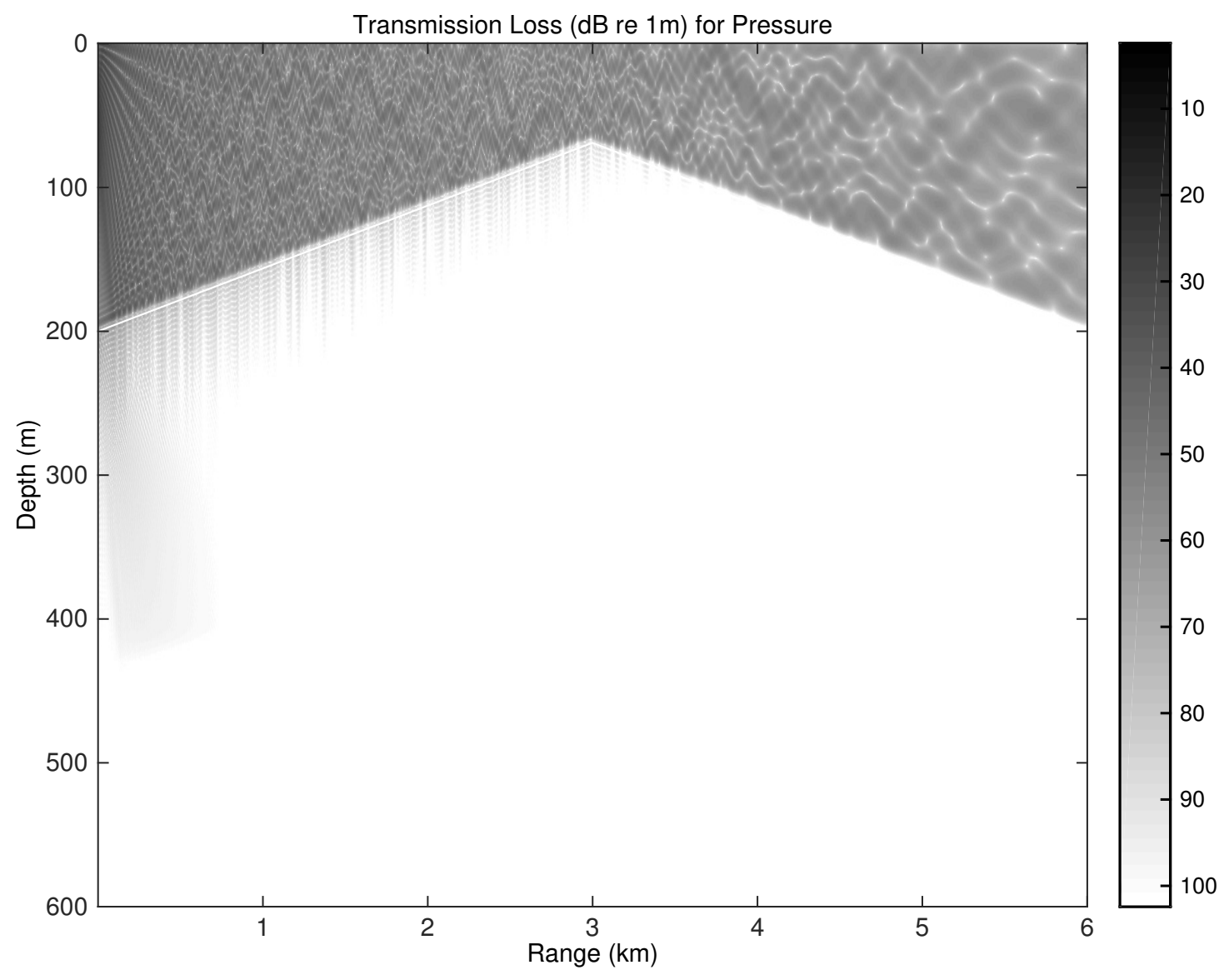

Figure 4.9: MMPE-predicted complex pressure field showing losses in the pseudorigid basement 


\subsection{Comparison to 3D Finite Element Solution}

As a final means of verification of the proposed coupled-mode solution, a comparison was made to a three-dimensional Finite Element Model (FEM) constructed by the Applied Research Laboratories of the University of Texas at Austin (ARL-UT). This model utilizes out-of-plane wavenumber decomposition techniques, which involve a series of two-dimensional models to calculate the fully

three-dimensional acoustic field for longitudinally invariant environments $[5,6]$. The geometry of the FEM-modeled environment consisted of an underwater sea ridge, which was constructed by extending the two-dimensional seamount scenario depicted in Figure (4.5) infinitely in a second horizontal dimension. As such, comparison to the FEM was achieved by taking a two-dimensional slice in-plane with the acoustic source. Figures (4.10a)-(4.10b) provide a comparison of transmission loss estimates produced by the two-dimensional (i.e. COUPLE and ASCM) solutions and the three-dimensional FEM.

Strong agreement was found between all three propagation solutions. The ASCM and FEM solutions showed a stronger peak-to-peak agreement in the vicinity of mode cutoff locations. Conversely, the ASCM and COUPLE solutions showed stronger agreement at end range. Based on communication with investigators at ARL-UT, this disagreement at $+4.5 \mathrm{~km}$ was suggested to be due to a difference in the predicted mode 1 energy on the downslope half of the ridge. Differences between the solutions may also be attributed to three-dimensional modal diffraction and the backwards traveling field, neither of which were included in the twodimensional solutions. Despite these differences, the presented results suggest that the ASCM solution provides an accurate estimate of the acoustic field in threedimensional environments with significant bathymetric variation. 


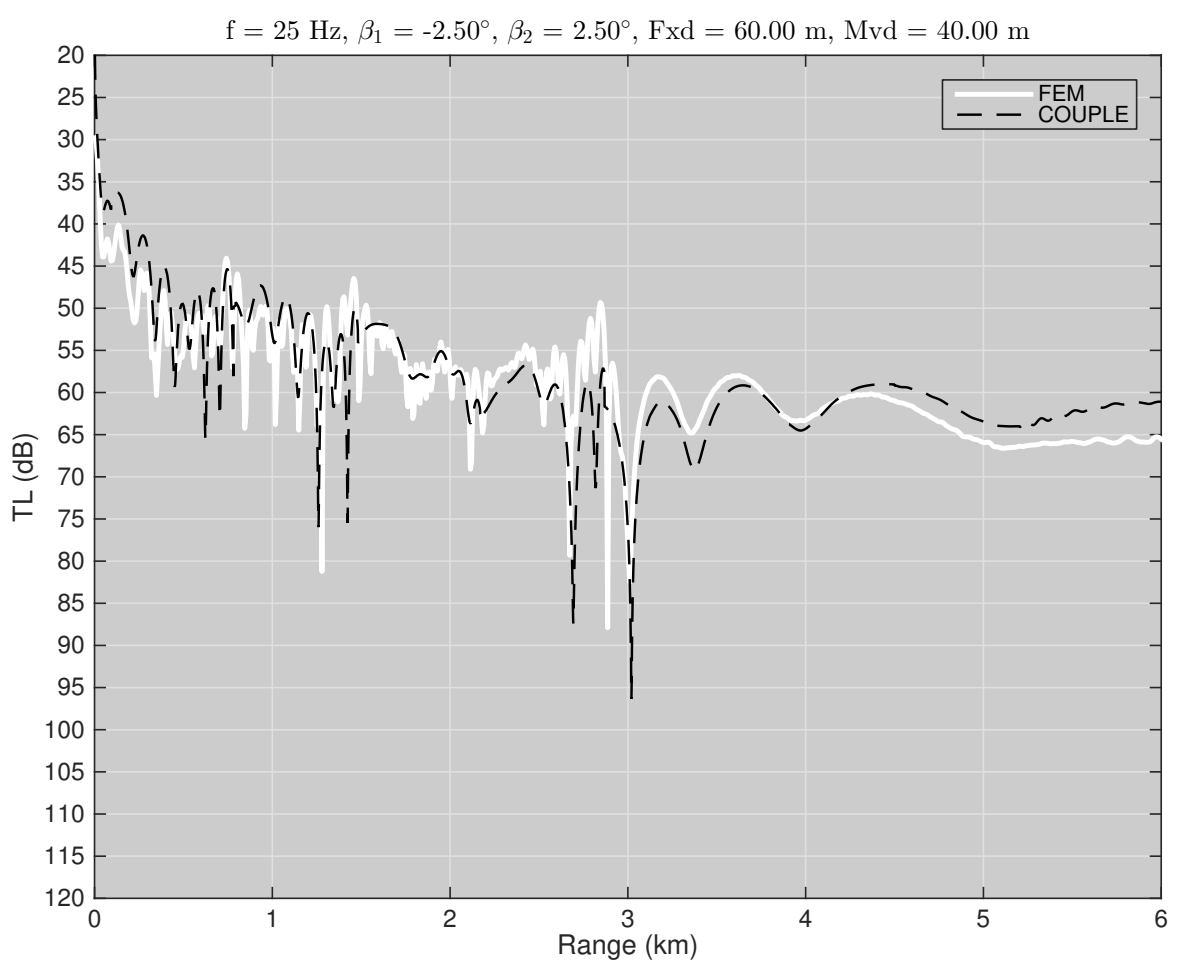

(a) COUPLE vs. FEM Transmission Loss Comparison

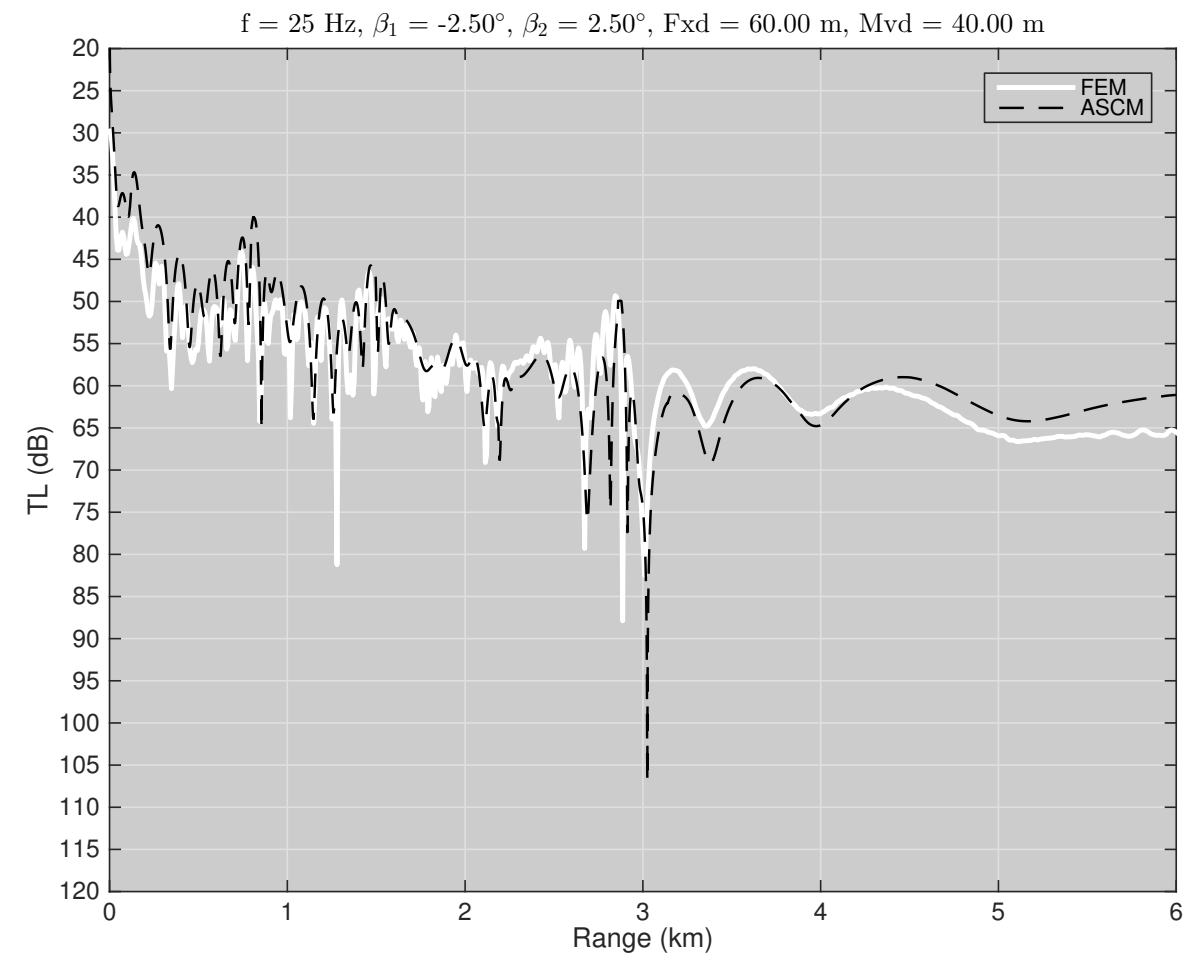

(b) ASCM vs. FEM Transmission Loss Comparison

Figure 4.10: Comparison of 2D and 3D solutions for in-plane transmission loss over a three-dimensional underwater sea ridge 


\subsection{Discussion}

Strong agreement was found between the transmission loss curves produced by the ASCM and COUPLE models for all seven cases depicted in Figures (4.1)(4.7). Small discrepancies can be found between the two solutions for the upslope propagation scenario depicted in Figure (4.1). These discrepancies correspond to areas where the transmission coefficients in the ASCM calculation have been corrected for mode turnaround points as discussed in Section (3.8). This same disagreement is found in the first half of the transmission loss curve for the 2D seamount scenario depicted in Figure (4.5). This is due to the fact that the scenario in Figure (4.5) was created by replicating and reflecting the bottom slope for the upslope scenario.

Some disagreement between the two models is also found in the vicinity of the valley of the 2D canyon case depicted in Figure (4.6). This disagreement is again contributed to the mode turnaround issue discussed in Section (3.8). In the case of the $2 \mathrm{D}$ canyon, severe mode stripping occurs from $2.5 \mathrm{~km}$ to $3.5 \mathrm{~km}$. Over this range interval higher order modes transition into a propagating state as acoustic energy propagates downslope and the increased waveguide depth moves away from the cutoff depths of these modes. These newly propagating modes are, however, quickly cutoff again as the energy radiates out of the valley of the canyon and up the second slope.

The results depicted in Figure(4.7) for the general multi-slope propagation scenario offers particular promise for the practical application of the presented theory. In this scenario strong agreement is found between the ASCM and COUPLE solutions for a highly variable bottom depth profile over a large range interval. These results support the previous conjecture that any bottom depth profile may be approximated by a series of connected wedge subenvironments. Moreover, the 
asymptotic solution (i.e. ASCM) is seen to perform similarly to the full-integral benchmark solution (i.e. COUPLE) for this more complicated environment. This would suggest that the ASCM solution has the potential to offer comparable accuracy to the benchmark solution at less computational cost. Strong agreement with the Parabolic Equation and three-dimensional Finite Element Model solutions discussed in Sections (4.3) and (4.4) further support the efficacy of the presented asymptotic stepwise coupled-mode approach.

\section{List of References}

[1] MATLAB, version 8.4.0 (R2014b). Natick, Massachusetts: The MathWorks Inc., 2014.

[2] R. B. Evans, "A coupled mode solution for acoustic propagation in a waveguide with stepwise depth variations of a penetrable bottom," Journal of the Acoustical Society of America, vol. 74, no. 1, pp. 188-195, 1983.

[3] F. B. Jensen, W. A. Kuperman, M. B. Porter, and H. Schmidt, Computational Ocean Acoustics, 1st ed. New York: Springer-Verlag, 2000.

[4] K. B. Smith, "Convergence, stability, and variability of shallow water acoustic predictions using a split-step fourier parabolic equation model," Journal of Computational Acoustics, vol. 9, no. 1, pp. 243-285, 2001.

[5] M. J. Isakson and N. P. Chotiros, "Finite element modeling of reverberation and transmission loss in shallow water waveguides with rough boundaries," Journal of the Acoustical Society of America, vol. 129, no. 3, pp. 1273-1279, 2011.

[6] M. J. Isakson, B. Goldsberry, and N. P. Chotiros, "A three-dimensional, longitudinally-invariant finite element model for acoustic propagation in shallow water waveguides," Journal of the Acoustical Society of America, vol. 136, no. 3, pp. EL206-EL211, 2014. 


\section{CHAPTER 5}

\section{Conclusion}

An asymptotic theory for estimating non-adiabatic acoustic mode coupling in horizontally-variable shallow water environments has been presented. This theory is based on a novel discretization of the range-dependent acoustic medium, which allows for the computation of mode coupling via a range-expanded normal mode inner product. Energy transfer due to an acoustic excitation is accounted for via a difference equations approach that provides asymptotic solutions for transmission and reflection coefficients at interfaces throughout the discretized acoustic environment. This approach is unique in that coupling across these interfaces is calculated via closed-form algebraic expressions rather than inner product integrals. Moreover, the novel discretization approach associated with this method eliminates the eigenvalue search problem.

A computational model was developed in MATLAB [1] that implements this asymptotic stepwise coupled mode theory. Transmission loss curves produced by this model for several test cases involving environments with range-dependent bottom depth were compared to those produced by a benchmark model for rangedependent problems developed by Evans [2]. Strong agreement was found between the two models for all cases considered. For cases in which discrepancies existed between the two solutions, these differences were attributed to an eigenvalue lag distance that is inherent to the stepwise approach of the asymptotic solution in the vicinity of mode turnaround points, as discussed in Section (3.8). Additional verification of the proposed theory was obtained through comparison to a Parabolic Equation solution in Sections (4.3) and to a three-dimensional Finite Element Model in Section (4.4). 
As discussed in Section (3.3), application of the discretization technique associated with the presented theory to general environments necessitates thought. This is due to the fact that one must construct a set of subenvironments that capture the range-dependence of the general environment via closed-form expressions for the eigenfunctions and eigenvalues associated with the acoustic modes of vibration of the medium. As shown in Section (3.3), range-dependence due to horizontally-variable bottom depth may be approximated via a series of acoustic wedges for which the desired closed-form expressions for the eigenfunctions and eigenvalues have been stated. It is plausible that range-dependence due to sound speed could be captured in a similar fashion by constraining each wedge subenvironment to ranges of constant gradient in the sound speed profile. This would, however, require further analysis including re-derivation of the closed-form expressions for the terms of the range-expanded normal mode inner product given by Equations (3.21)-(3.26).

The inclusion of range-dependent sound speed into the current model would allow for the modeling of more realistic environments and the ability to make meaningful runtime comparisons with other solutions. Despite the omission of these types of comparisons in the present work, it is the opinion of the author that appreciable gains in runtime performance will be achieved due to the ability to compute mode coupling via the evaluation of closed-form algebraic expressions instead of inner product integrals. This is particularly evident if one considers the number of range-independent steps necessary to accurately approximate a general range-dependent environment. As non-adiabatic mode coupling must be accounted for at the interfaces separating each of these steps, the multiplicity of the aforementioned coupling calculation is significant.

There is room for improvement in the handling of the mode turnaround issue 
discussed in Section (3.8). While the proposed moving average solution successfully eliminates the artificial peaks introduced into the transmission loss curves associated with this issue, the largest discrepancies between the asymptotic and benchmark models occurred at ranges associated with mode cutoff. One potential improvement on this issue may be to simply apply a more sophisticated smoothing filter to the eigenvalue value ratio appearing in the transmission coefficient matrix in Equation (3.81). Another approach would be to develop an adaptive range-step algorithm that reduces range step size in the propagation code in the neighborhood of mode cutoff depths. This would effectively reduce the eigenvalue lag distance discussed in Section (3.8) and therefore potentially reduce or eliminate the discontinuities in the transmission coefficients associated with mode turnaround.

\section{List of References}

[1] MATLAB, version 8.4.0 (R2014b). Natick, Massachusetts: The MathWorks Inc., 2014.

[2] R. B. Evans, "A coupled mode solution for acoustic propagation in a waveguide with stepwise depth variations of a penetrable bottom," Journal of the Acoustical Society of America, vol. 74, no. 1, pp. 188-195, 1983. 


\section{APPENDIX}

\section{Note on the Effects of Range-Dependent Bathymetry on Modal Group Velocities}

Mode coupling is a process that affects the amplitudes of the eigenfunctions associated with the normal modes of propagation of an acoustic waveguide. As such, it does not have a direct impact on modal phase or group velocities, which are functions of the eigenvalues rather than the eigenfunctions. This said, both mode coupling and perturbations in modal velocities originate due to environmental range-dependence. As such, these two physical phenomena share a common source and are equally important in the understanding of wave propagation in horizontally-variable acoustic media.

While conducting the research and development of the presented coupledmode theory and associated model a numerical study was performed on the effects of horizontally-variable bottom depth on modal group velocities. To perform this study, numerical models were constructed for three isovelocity waveguides: a flatbottom waveguide, an acoustic wedge with a bottom slope angle of $-1^{\circ}$, and an acoustic wedge with a bottom slope angle of $-2^{\circ}$. The sound speed in each waveguide was chosen to be $1500 \mathrm{~ms}^{-1}$ and the maximum waveguide depth was chosen to be $200 \mathrm{~m}$. To assess the relationship between frequency and group speed an evenly-spaced frequency band from $40 \mathrm{~Hz}$ to $200 \mathrm{~Hz}$ was generated using a $5 \mathrm{~Hz}$ frequency spacing.

Group velocities were computed for all modes and all frequencies at $1 \mathrm{~m}$ range increments across a distance of $3 \mathrm{~km}$ in each waveguide. The mean of these samples was then calculated, providing one range-averaged group velocity per mode, per

frequency for each of the three waveguides. Note that for a given mode in the wedge waveguides, range increments at which the mode became cutoff and thus its 
eigenvalue became purely imaginary were not included in the averaging process. Figures (A.1)-(A.3) provide depictions of the range-averaged group velocities and grazing angles for modes 1,3 , and 7 for the three sample waveguides.

As is evident from the figures, increasing the bottom slope (i.e. reducing the waveguide depth with range) results in a reduction in modal group speeds across all frequencies. This "slowing down" of the modal envelope is most pronounced at lower frequencies and smallest at higher frequencies. This phenomenon can also be connected to mode grazing angle. That is, increasing the bottom slope of the waveguide increases the mode grazing angles at all frequencies. As is the case for group velocity, the perturbation in mode grazing angle is most pronounced at lower frequencies and least pronounced at higher frequencies. If one considers the relationship between frequency, waveguide depth, and mode cutoff, the effects illustrated in Figures (A.1)-(A.3) suggest that the closer a mode comes to its cutoff conditions (i.e. steep-angle and imaginary eigenvalue) the more "slowed down" it becomes and therefore the greater the perturbation in its group speed relative to the flat-bottom case.

The findings of this experiment are particularly significant for shallow water applications of mode theory in which modal velocities are considered at low frequencies. For example, models involving group velocity are often used in geoacoustic inversion applications to estimate sediment properties. In these applications it is desirable to excite the normal modes of the acoustic waveguide at steep angles (i.e. at low frequencies near modal cutoffs) to maximize sediment penetration. For flat or nearly flat waveguides it has been shown that the effects of horizontally-variable sound speed on geoacoustic inversion is negligible as the perturbation in modal group velocities due to this variability is most pronounced at higher frequencies [1]. As such, the assumption of range-independence is often 
made in these applications. The results shown here suggest that if the waveguide has significant bathymetric variability, the perturbation in modal group velocity is most pronounced in the vicinity of mode cutoff and therefore the assumption of range-independence could introduce considerable error into the inversion process. 


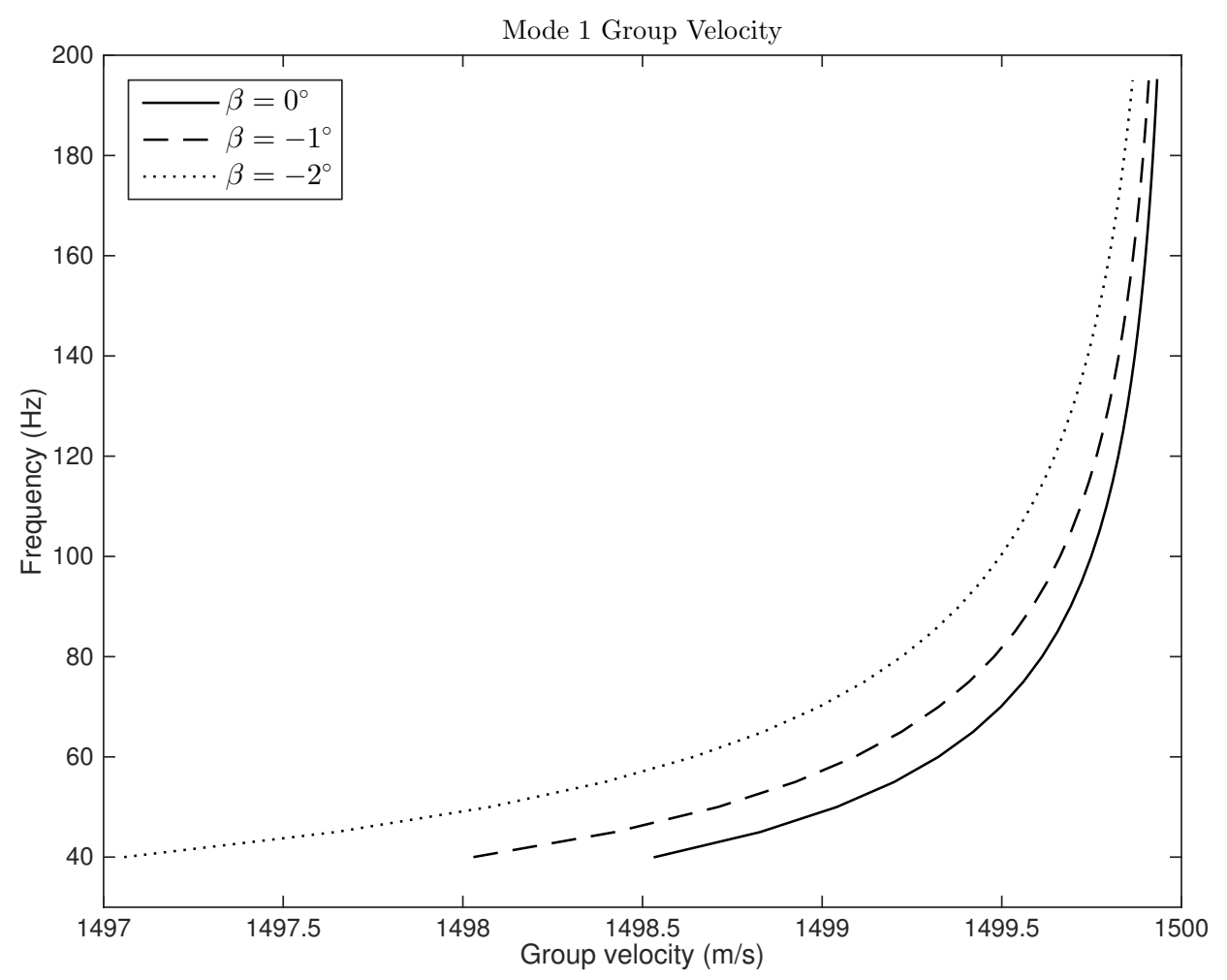

(a)

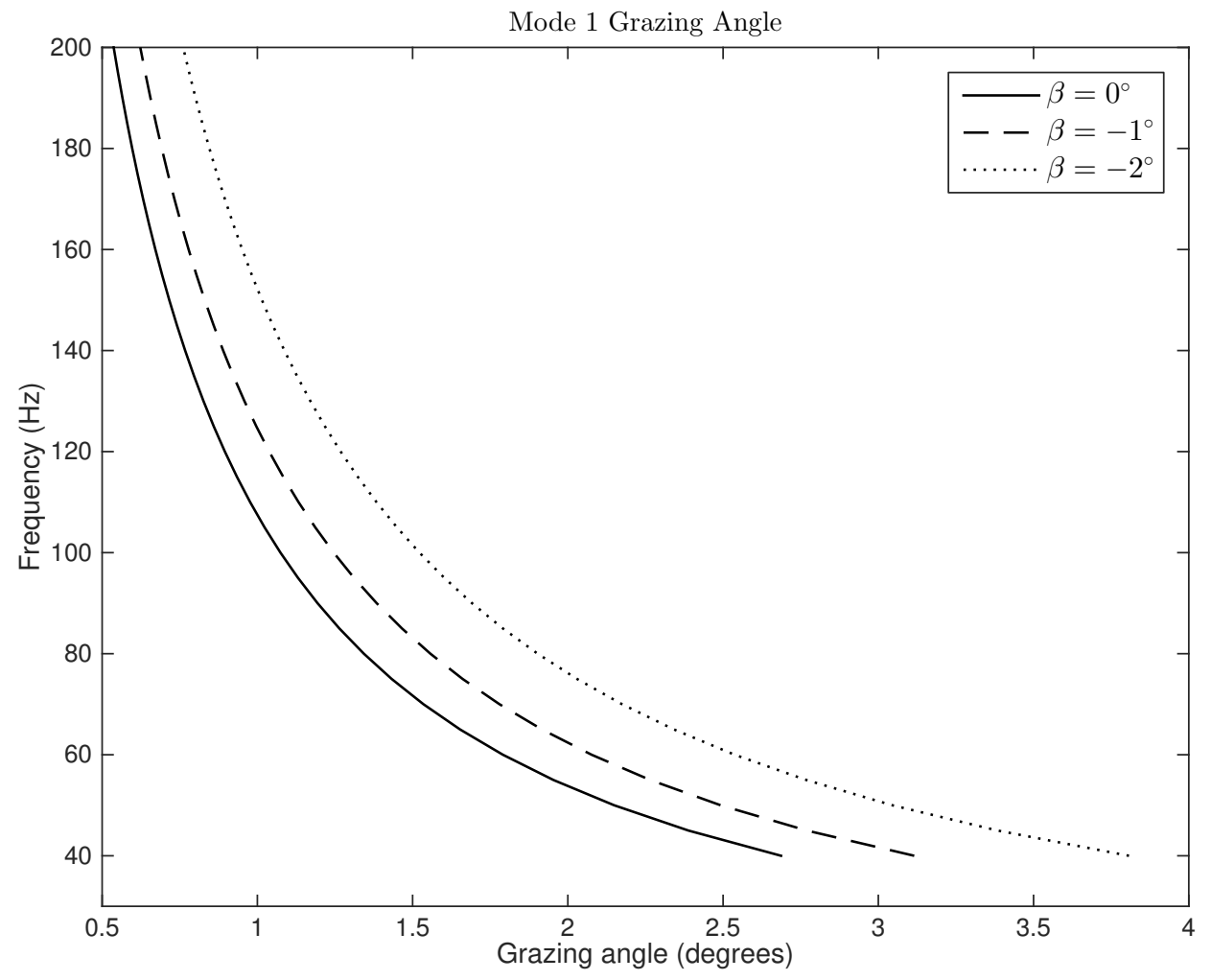

(b)

Figure A.1: Comparison of mode 1 (a) group velocity and (b) grazing angle across three waveguides with varied bottom slope angle 


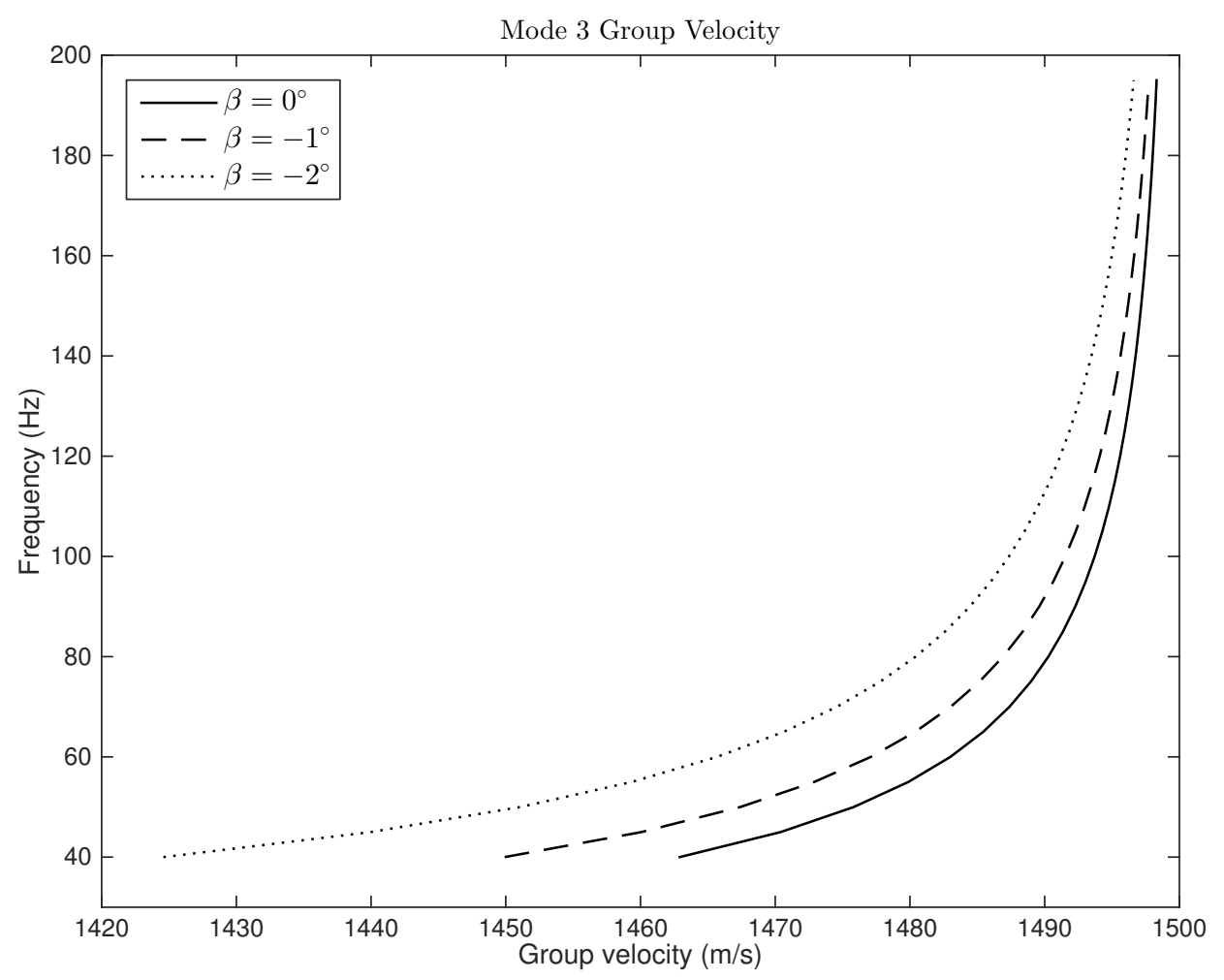

(a)

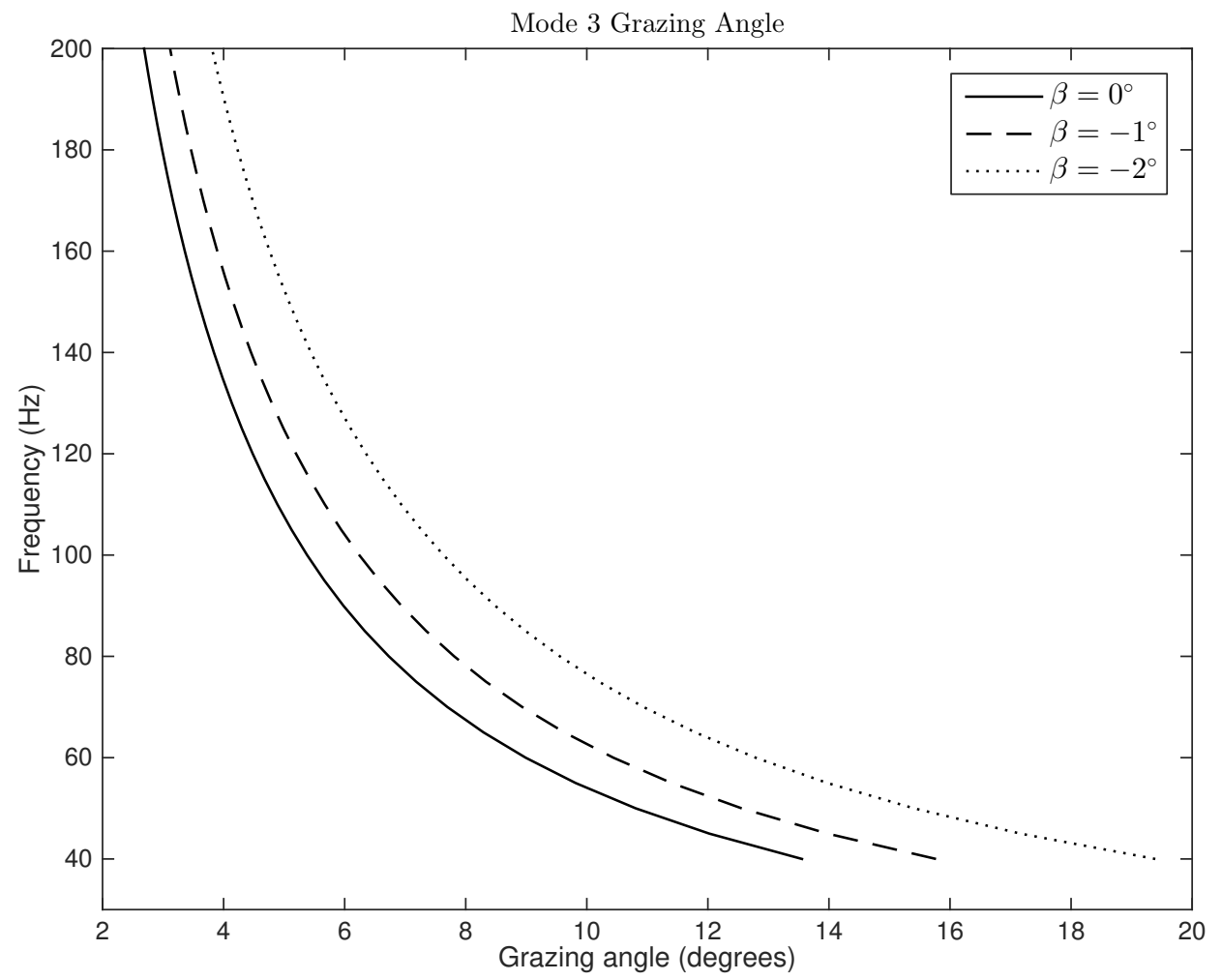

(b)

Figure A.2: Comparison of mode 3 (a) group velocity and (b) grazing angle across three waveguides with varied bottom slope angle 


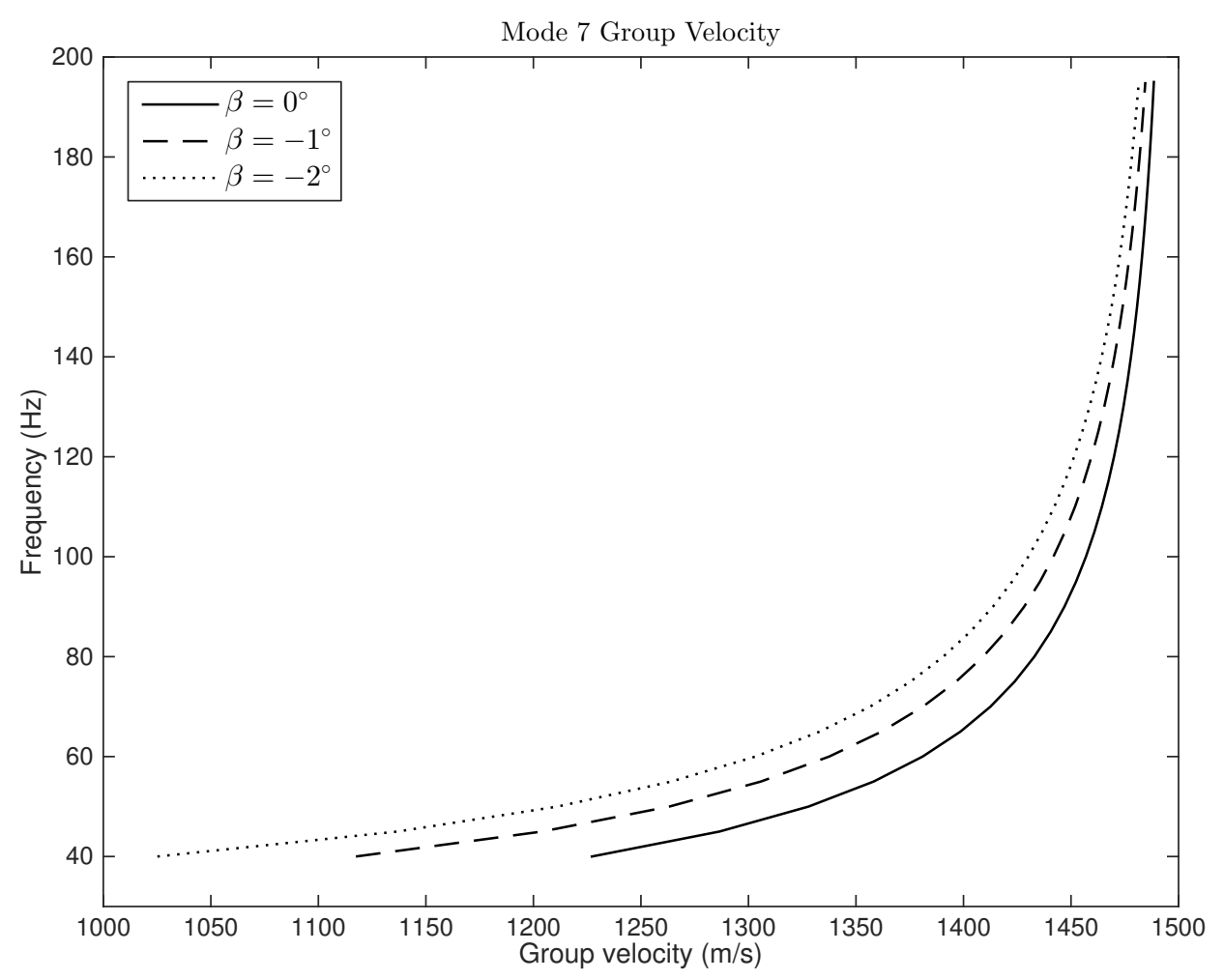

(a)

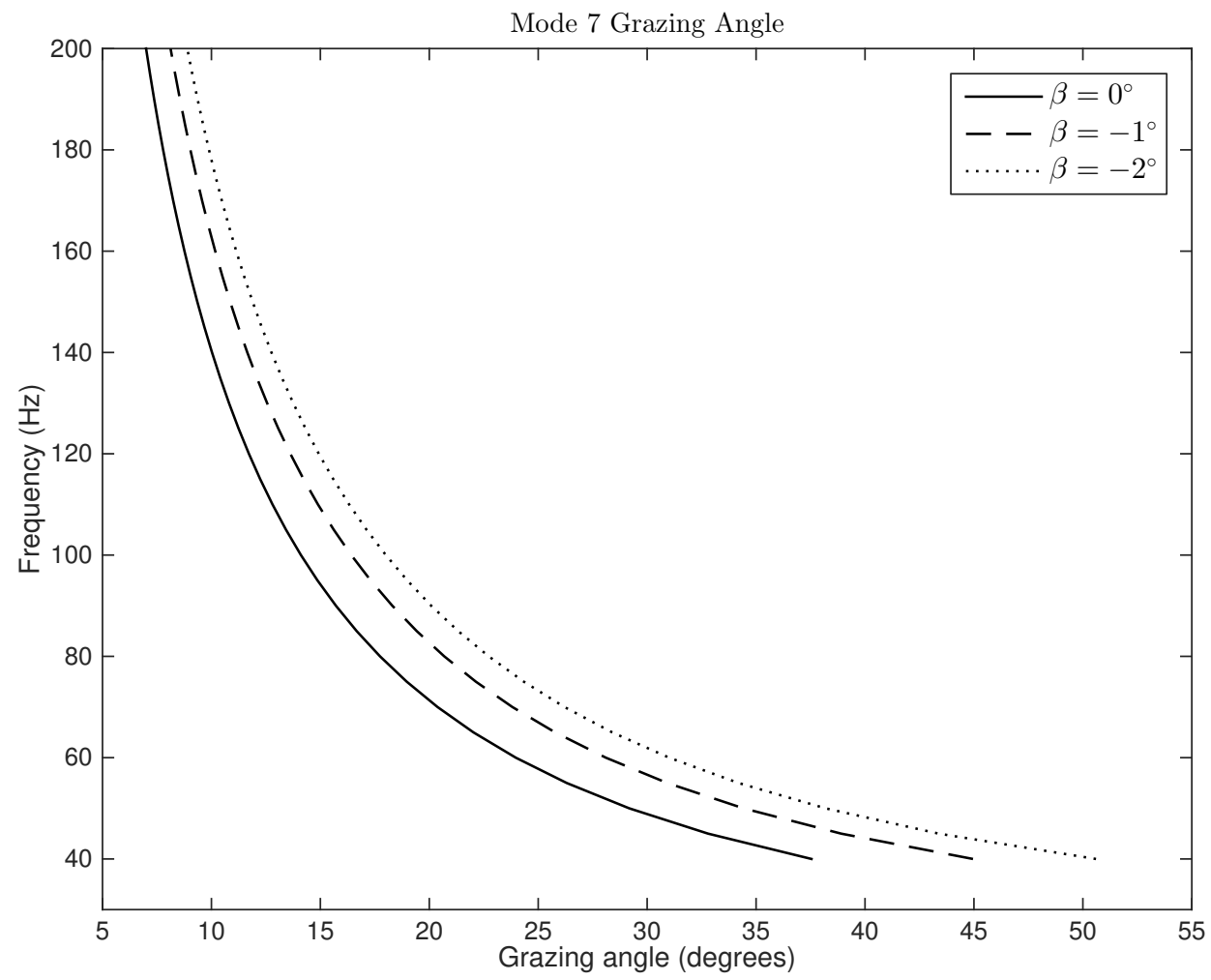

(b)

Figure A.3: Comparison of mode 7 (a) group velocity and (b) grazing angle across three waveguides with varied bottom slope angle 


\section{List of References}

[1] G. R. Potty, J. H. Miller, J. F. Lynch, and K. B. Smith, "Tomographic inversion for sediment parameters in shallow water," Journal of the Acoustical Society of America, vol. 108, no. 3, pp. 973-986, 2000. 


\section{BIBLIOGRAPHY}

Boyles, C. A., "Coupled mode solution for a cylindrically symmetric oceanic waveguide with a range and depth dependent refractive index and a time varying rough sea surface," Journal of the Acoustical Society of America, vol. 73, no. 3, pp. 800-805, 1983.

Boyles, C. A., Acoustic Waveguides: Applications to Oceanic Science. New York: Wiley-Interscience, 1984.

Brown, J. W. and Churchill, R. V., Complex Variables and Applications, 7th ed. New York: McGraw-Hill, 2003.

Buckingham, M. J., "Theory of three-dimensional acoustic propagation in a wedgelike ocean with a penetrable bottom," Journal of the Acoustical Society of America, vol. 82, no. 1, pp. 198-210, 1987.

Chiu, C.-S., Miller, J. H., and Lynch, J. F., "Forward coupled-mode propagation modeling for coastal acoustic tomography," Journal of the Acoustical Society of America, vol. 99, no. 2, pp. 793-802, 1996.

Clark, C. A., "Acoustic wave propagation in horizontally variable media," IEEE Journal of Oceanic Engineering, vol. 30, no. 1, pp. 188-197, 2005.

Clark, C. A. and Smith, K. B., "An efficient normal mode solution to wave propagation prediction," IEEE Journal of Oceanic Engineering, vol. 33, no. 4, pp. 462-476, 2008.

de Brujin, N. G., Asymptotic Methods in Analysis. New York: Dover Publications, 1981.

Desaubies, Y., Chiu, C.-S., and Miller, J. H., "Acoustic mode propagation in a range-dependent ocean," Journal of the Acoustical Society of America, vol. 80, no. 4, pp. 1148-1160, 1986.

Evans, R. B., "A coupled mode solution for acoustic propagation in a waveguide with stepwise depth variations of a penetrable bottom," Journal of the Acoustical Society of America, vol. 74, no. 1, pp. 188-195, 1983.

Fawcett, J. A., Westwood, E. K., and Tindle, C. T., "A simple coupled wedge mode propagation method," Journal of the Acoustical Society of America, vol. 98, no. 3, pp. 1673-1681, 1995.

Frisk, G. V., Ocean and Seabed Acoustics: A Theory of Wave Propagation. New Jersey: Prentice-Hall, 1994. 
Gillette, G., "Coupled modes in a waveguide with a range-dependent rigid basement," Journal of the Acoustical Society of America, vol. 95, no. 1, pp. 187200, 1994.

Godin, O. A., "A note on differential equations of coupled-mode propagation in fluids," Journal of the Acoustical Society of America, vol. 103, no. 1, pp. 159168, 1998.

Hardin, R. H. and Tappert, F. D., "Applications of the split-step fourier method to the numerical solution of nonlinear and variable coefficient wave equations," SIAM Rev., vol. 15, p. 423, 1973.

Higham, C. J. and Tindle, C. T., "Coupled perturbed modes and internal solitary waves," Journal of the Acoustical Society of America, vol. 113, no. 5, pp. 2515-2522, 2003.

Higham, C. J. and Tindle, C. T., "Coupled perturbed modes over a sloping penetrable bottom," Journal of the Acoustical Society of America, vol. 114, no. 6, pp. 3119-3124, 2003.

Isakson, M. J. and Chotiros, N. P., "Finite element modeling of reverberation and transmission loss in shallow water waveguides with rough boundaries," Journal of the Acoustical Society of America, vol. 129, no. 3, pp. 1273-1279, 2011.

Isakson, M. J., Goldsberry, B., and Chotiros, N. P., "A three-dimensional, longitudinally-invariant finite element model for acoustic propagation in shallow water waveguides," Journal of the Acoustical Society of America, vol. 136, no. 3, pp. EL206-EL211, 2014.

Jensen, F. B., Kuperman, W. A., Porter, M. B., and Schmidt, H., Computational Ocean Acoustics, 1st ed. New York: Springer-Verlag, 2000.

Kampanis, N. A. and Dougalis, V. A., "A finite element code for the numerical solution of the Helmholtz equation in axially symmetric waveguides with interfaces," Journal of Computational Acoustics, vol. 7, no. 2, pp. 83-110, 1999.

Leibiger, G. A., "Wave propagation in an inhomogeneous medium with slow spatial variation," Ph.D. dissertation, Stevens Institute of Technology, Castle Point, Hoboken, New Jersey, 1968.

MATLAB, version 8.4.0 (R2014b). Natick, Massachusetts: The MathWorks Inc., 2014.

Milder, D. M., "Ray and wave invariants for sofar channel propagation," Journal of the Acoustical Society of America, vol. 46, no. 5, pp. 1259-1263, 1969. 
Pierce, A. D., "Extension of the method of normal modes to sound propagation in an almost-stratified medium," Journal of the Acoustical Society of America, vol. 37, no. 1, pp. 19-27, 1964.

Potty, G. R., Miller, J. H., Lynch, J. F., and Smith, K. B., "Tomographic inversion for sediment parameters in shallow water," Journal of the Acoustical Society of America, vol. 108, no. 3, pp. 973-986, 2000.

Primack, H. and Gilbert, K. E., "A two-dimensional downslope propagation model based on coupled wedge modes," Journal of the Acoustical Society of America, vol. 90, no. 6, pp. 3254-3262, 1991.

Smith, K. B., "Convergence, stability, and variability of shallow water acoustic predictions using a split-step fourier parabolic equation model," Journal of Computational Acoustics, vol. 9, no. 1, pp. 243-285, 2001.

Stotts, S. A., "Coupled-mode solutions in generalized ocean environments," Journal of the Acoustical Society of America, vol. 111, no. 4, pp. 1623-1643, 2002.

Tindle, C. T., O’Driscoll, L. M., and Higham, C. J., "Coupled mode perturbation theory of range dependence," Journal of the Acoustical Society of America, vol. 108, no. 1, pp. 76-83, 2000.

Watson, G. N., A Treatise on the Theory of Bessel Functions, 2nd ed. New York: Cambridge University Press, 1995.

White, F. M., Fluid Mechanics, 6th ed. New York: McGraw-Hill, 2006. 